\title{
A priori estimates and existence for elliptic equations with gradient dependent terms
}

\author{
Nathalie Grenon, François Murat and Alessio Porretta
}

\begin{abstract}
We consider, in a bounded domain $\Omega \subset \mathbb{R}^{N}$, a class of nonlinear elliptic equations in divergence form as

$$
\begin{cases}\alpha_{0} u-\operatorname{div}(a(x, u, D u))=H(x, u, D u) & \text { in } \Omega, \\ u=0 & \text { on } \partial \Omega\end{cases}
$$

where $\alpha_{0} \geq 0$, the second order part is a coercive, pseudomonotone operator of Leray-Lions type in the Sobolev space $W_{0}^{1, p}(\Omega), p>1$, and the function $H$ grows at most like $|D u|^{q}+f(x)$, with $p-1<q<p$. Assuming $f(x)$ to belong to an (optimal) Lebesgue class $L^{m}$, with $m<\frac{N}{p}$, we prove a priori estimates and existence of solutions, discussing several ranges of the exponents $m, q$ and $p$ which include cases of singular data ( $L^{1}$ data or measures). The obtention of a priori estimates is not straightforward because of the "superlinear"character of the first order terms. To this purpose we use a new approach, generalizing the method introduced in our note [29]. We complete the results known in the previous literature where either $q \leq p-1$ or $m \geq \frac{N}{p}$.
\end{abstract}

Mathematics Subject Classification (2010): 35J60 (primary); 35J25, 35R05, 35Dxx (secondary).

\section{Introduction}

Let $\Omega$ be a bounded domain in $\mathbb{R}^{N}, N \geq 1$. In this paper we will deal with the following class of nonlinear elliptic equations in divergence form

$$
\begin{cases}-\operatorname{div}(a(x, u, D u))+a_{0}(x, u)=H(x, u, D u) & \text { in } \Omega, \\ u=0 & \text { on } \partial \Omega\end{cases}
$$

where the operator $-\operatorname{div}(a(x, u, D u))$ is a bounded, coercive and pseudomonotone operator of Leray-Lions type in the Sobolev space $W_{0}^{1, p}(\Omega), p>1, a_{0}$ satisfies 
$a_{0}(x, u) u \geq 0, H(x, u, D u)$ is a lower order term which satisfies $|H(x, u, D u)| \leq$ $\gamma|D u|^{q}+f(x)$, where $p-1<q<p$, and the function $f$ belongs to some Lebesgue space $L^{m}(\Omega)$ to be specified later.

The simplest model that we have in mind is the following example

$$
\left\{\begin{array}{l}
-\Delta_{p}(u)+\alpha_{0} u=\gamma|D u|^{q}+f(x) \text { in } \Omega, \\
u=0 \text { on } \partial \Omega
\end{array}\right.
$$

where $-\Delta_{p}(u)=-\operatorname{div}\left(|D u|^{p-2} D u\right)$ is the $p$-Laplace operator and $\alpha_{0} \geq 0$. If $p=2$, problem (1.2) is also referred to as a viscous Hamilton-Jacobi equation.

Let us now discuss the restrictions $p-1<q<p$ on the growth of the righthand side with respect to $|D u|$. We confine now our discussion to the case $p<N$, even if we also deal with the case $p \geq N$ in the present paper.

The case where $0 \leq q<p-1$ is well known. Indeed, in this case an a priori estimate for every solution of (1.1) with $u \in W_{0}^{1, p}(\Omega)$ is easily obtained using $u$ as test function, and existence follows; this is part of the general theory of pseudomonotone operators of J. Leray and J.-L. Lions, see e.g. [33]. In contrast, the limit case $q=p-1$ presents important difficulties when $\gamma$ is large. Indeed, while for small $\gamma$ the operator $-\operatorname{div}(a(x, u, D u))-H(x, u, D u)$ is coercive, this is no more the case for large $\gamma$; nevertheless this problem has been solved first by G. Bottaro and M. E. Marina [19] in the linear case, and then by various authors in the nonlinear case, see e.g. [8] and [23].

On the other hand, we confine here ourselves to the case $q \leq p$ in order for the growth of $H(x, u, D u)$ not to exceed the natural energy $|D u|^{p}$ of the principal part of the operator, which in particular allows us to define $H(x, u, D u)$ as a function of $L^{1}(\Omega)$, and thus as a distribution, whenever $u \in W_{0}^{1, p}(\Omega)$. The case $q>p$ is completely different and largely open; some results can be found in $[4,20,34]$ and [35].

The limit case $q=p$ has been considered in many papers, first in the case where $f \in L^{m}(\Omega)$ with $m>\frac{N}{p}$, allowing one to prove a priori estimates for (and existence of) solutions of (1.1) in $W_{0}^{1, p}(\Omega) \cap L^{\infty}(\Omega)$, see e.g. [15-17] and [37]. The case where $q=p$ and $m=\frac{N}{p}$ was then studied in [25,26] (if $\alpha_{0}=0$ ) and [21] (if $\alpha_{0}>0$ ). In those papers the authors prove a priori estimates for (and existence of) solutions in $W_{0}^{1, p}(\Omega)$ which are not bounded in general but satisfy $\exp \left(\frac{\gamma}{p-1}|u|\right)-1 \in W_{0}^{1, p}(\Omega)$. The latter regularity comes, roughly speaking, from the underlying change of unknown function $w=\left[\exp \left(\frac{\gamma}{p-1}|u|\right)-1\right] \operatorname{sign} u$ which allows one to cancel the term $\gamma|D u|^{p}$ in the case of equation (1.2) with $q=p$ (see [25] and [26]). Let us also mention, without further comments, the case where the right-hand side $H(x, u, D u)$ has a good sign, namely $H(x, u, D u)=$ $f(x)-g(x, u, D u)$ with $g(x, s, \xi) s \geq 0$, see e.g. [7].

Thus, we deal here with the range $p-1<q<p$. Of course, if $f \in L^{\frac{N}{p}}(\Omega)$, the results known in the case $q=p$ apply immediately to $q<p$. Therefore, 
we consider the case of $f \in L^{m}(\Omega)$ with $m<\frac{N}{p}$, and we obtain results for the "optimal" exponent $m$, which depends on $q$. We provide here both existence results and, what is actually more important, a priori estimates for solutions.

The results of the present paper generalize and develop our note [29]. Similar results were obtained in [24] and very recently in [2] using symmetrization methods (see also [3] for the case $p=2$ ). We finally refer to [18] for results on some equation close to (1.2) under different assumptions.

\subsection{The model problem: statement of the results in the case of solutions of finite energy}

When dealing with the problem of existence of solutions for problems like (1.2), some necessary conditions are required on the data. Such necessary conditions are derived in a sharp way in [1] and [30] for $p=2$ and $\alpha_{0}=0$ : when specialized to the class of Lebesgue spaces, they correspond to $f \in L^{m}(\Omega)$ with $m \geq \frac{N(q-1)}{q}$ (assuming $q \geq \frac{N}{N-1}$ in order to have $m \geq 1$ ), and $\|f\|_{L^{m}(\Omega)}$ sufficiently small. In the case of arbitrary $p$, similar necessary conditions may be derived and lead to

$$
f \in L^{m}(\Omega), \quad \text { with } m \geq \frac{N(q-(p-1))}{q},
$$

(assuming $q \geq \frac{N(p-1)}{N-1}$ in order to have $m \geq 1$ ), and in addition, if $\alpha_{0}=0$, to $\|f\|_{L^{m}(\Omega)}$ sufficiently small.

The reader may try to convince himself that condition (1.3) is natural by looking at the linear case (i.e. $p=2$ )

$$
-\Delta u=\gamma|D u|^{q}+f(x) .
$$

By standard elliptic regularity ${ }^{1}, \Delta u \in L^{m}(\Omega)$ implies $u \in W^{2, m}(\Omega)$, which in turn, by Sobolev embedding, implies $|D u| \in L^{m^{*}}(\Omega), m^{*}=\frac{N m}{N-m}$. Thus when $f \in L^{m}(\Omega)$ one can expect that $|D u|^{q}$ belongs both to $L^{m}(\Omega)$ and to $L^{\frac{m^{*}}{q}}(\Omega)$, which leads to take $m=\frac{m^{*}}{q}$. When $p=2$ this is the equality in condition (1.3).

Henceforth, we assume condition (1.3), and more precisely, when we deal with existence results, $m=\frac{N(q-(p-1))}{q}$ (which is the weakest condition in bounded domains). Note that when $q=p$ one recovers $f \in L^{\frac{N}{p}}(\Omega)$, as in the above cited literature; however, if $q<p$, this choice allows us to take data $f$ in a larger Lebesgue space, suitably depending on $q$, which was not considered previously. Moreover, when $\alpha_{0}=0$ (no zero-th order term in the left-hand side), we will assume a size condition on $\|f\|_{L^{m}(\Omega)}$ (as mentioned before, this is necessary) but such a condition will not be required whenever $\alpha_{0}>0$.

${ }^{1}$ In this paper, dealing with operators with only measurable bounded coefficients, we will never make use of the $W^{2, m}$ regularity. We only use it for similar short examples like this one where we consider the Laplace operator in order to clarify some necessary condition on the data. 
Let us now give the statement of our main results in the case of the model problem (1.2). For any $s>1$, we denote by $s^{\prime}=\frac{s}{s-1}$ the conjugate exponent of $s$, and, if $1 \leq s<N$, by $s^{*}=\frac{N s}{N-s}$ the Sobolev embedding exponent. In this subsection we restrict ourselves to the case $p<N$ and to the range $p-1+\frac{p}{N} \leq q$. This range is equivalent to $m=\frac{N(q-(p-1))}{q} \geq\left(p^{*}\right)^{\prime}$, which implies $L^{m}(\Omega) \subset$ $W^{-1, p^{\prime}}(\Omega)$, so that the data under consideration belong to the space $W^{-1, p^{\prime}}(\Omega)$ and solutions are in $W_{0}^{1, p}(\Omega)$. If $p=2$, these results have been proved in our note [29].

Theorem 1.1. Assume that $p-1+\frac{p}{N} \leq q<p$ and that $f \in L^{m}(\Omega)$ with $m=$ $\frac{N(q-(p-1))}{q}$. If either

(i) $\alpha_{0}>0$,

or

(ii) $\alpha_{0}=0$ and

$$
\gamma^{\frac{N-p}{p-q}}\|f\|_{L^{m}(\Omega)}^{\frac{p m^{\prime}}{p m^{\prime}-p^{*}}}<C^{*}
$$

where $C^{*}$ is a given constant depending only on $p, q$ and $N$, then every solution $u \in W_{0}^{1, p}(\Omega)$ of (1.2) such that

$$
|u|^{\sigma} \in W_{0}^{1, p}(\Omega), \quad \sigma=\frac{(N-p)(q-(p-1))}{p(p-q)},
$$

satisfies the estimate

$$
\|u\|_{W_{0}^{1, p}(\Omega)}+\left\||u|^{\sigma}\right\|_{W_{0}^{1, p}(\Omega)} \leq M,
$$

where $M$ is a constant that depends on $p, q, N, \alpha_{0}, \gamma,|\Omega|$ and $f$.

When $\alpha_{0}=0$, the constant $M$ in (1.7) depends only on $p, q, N, \gamma$ and $\|f\|_{L^{m}(\Omega)}$. When $\alpha_{0}>0$, the constant $M$ does not depend only on $\|f\|_{L^{m}(\Omega)}$ but it remains bounded when $f$ varies in a set which is bounded and equi-integrable in $L^{m}(\Omega)$.

We also observe that, using Sobolev embedding, estimate (1.7) implies an estimate for $|u|^{\sigma}$ in $L^{p^{*}}(\Omega)$, which means an estimate of $u$ in $L^{\left((p-1) m^{*}\right)^{*}}(\Omega)$ since $\sigma p^{*}=\left((p-1) m^{*}\right)^{*}$.

The a priori estimate (1.7) also holds for nonnegative subsolutions of (1.2) satisfying (1.6); in this case hypothesis (1.5) can be made upon $f^{+}$instead of $f$ (see Remark 3.4 below).

As usual, the a priori estimate (1.7) is a crucial step in the proof of the following existence result.

Theorem 1.2. Assume that the hypotheses of Theorem 1.1 hold true. Then there exists at least one solution $u \in W_{0}^{1, p}(\Omega)$ of (1.2) which also satisfies (1.6). 
As far as (1.6) is concerned, in the range $p-1+\frac{p}{N} \leq q<p$, we have $1 \leq \sigma<\infty$, hence (1.6) is stronger than simply requiring $u \in W_{0}^{1, p}(\Omega)$. Let us emphasize that the regularity class (1.6) is natural for such problems, as shown by the following well-known example.

Example 1.3. Let $\Omega=B_{1}(0)$ be the unit ball of $\mathbb{R}^{N}$. The function

$$
u(x)=C_{q}\left(|x|^{-\frac{2-q}{q-(p-1)}}-1\right)
$$

satisfies, for $q>p-1+\frac{p}{N}$ and for a suitable constant $C_{q}$,

$$
\left\{\begin{array}{l}
u \in W_{0}^{1, p}(\Omega), \\
-\Delta_{p} u=|D u|^{q} \quad \text { in } \mathcal{D}^{\prime}(\Omega),
\end{array}\right.
$$

but

$$
|u|^{\rho-1} u \in W_{0}^{1, p}(\Omega) \quad \text { if and only if } \rho<\sigma .
$$

This example (where $f=0$ ) proves that uniqueness does not hold for solutions of (1.2) in $W_{0}^{1, p}(\Omega)$, whereas the uniqueness of solutions in $W_{0}^{1, p}(\Omega)$ which also belong to the class (1.6) has been proved in [5] when $p=2$ and in [40] when $p>2$.

Moreover, this example proves that no bootstrap regularity holds for solutions which do not satisfy (1.6). In contrast, the following result proves that bootstrap regularity holds when starting in the class (1.6).

Theorem 1.4. Assume that the hypotheses of Theorem 1.1 hold and, in addition, that $f \in L^{s}(\Omega)$ with $s>m=\frac{N(q-(p-1))}{q}$. Let $u \in W_{0}^{1, p}(\Omega)$ be a solution of $(1.2)$ which satisfies (1.6). Then

(i) if $f \in L^{s}(\Omega), s>\frac{N}{p}$, then $u \in L^{\infty}(\Omega)$,

(ii) if $f \in L^{s}(\Omega), s=\frac{N}{p}$, then $u \in L^{t}(\Omega)$ for any $t<\infty$,

(iii) if $f \in L^{s}(\Omega), s<\frac{N}{p}$, then $u \in L^{\left(s^{*}(p-1)\right)^{*}}(\Omega)$,

with corresponding estimates depending on $\|f\|_{L^{s}(\Omega)}$.

Note that in the above result the source $f$ is taken in a Lebesgue space $L^{s}(\Omega)$ with $s$ bigger than the limiting value $m=\frac{N(q-(p-1))}{q}$. In this case, the result follows from an estimate of $|u|^{\tau-1} u$ in $W_{0}^{1, p}(\Omega)$ for some $\tau$ bigger than the exponent $\sigma$ in (1.6) (see Theorem 3.8 below).

Finally, let us point out that Theorems 1.1, 1.2 and 1.4 are nothing but the results, specialized to the model case of equation (1.2), which we prove later in more generality (Theorems 3.1, 3.7, 3.8). 


\subsection{Main ideas of the method}

Let us spend a few words on the method of proof of Theorem 1.1. The main novelty of our work relies on the proof of this a priori estimate, which is not obvious due to the superlinear growth of the right-hand side. Our proof, based on a continuity argument, is new with respect to previous results which were concerned with the case $q=p$ where a change of unknown can get rid of the "superlinear" term.

In order to explain the difficulties related to obtaining a priori estimates, let us consider example (1.4) where the principal part is the Laplace operator without zero-th order term. In this very special case, one is allowed to use the CalderonZygmund regularity, i.e. the $W^{2, m}$ regularity of the solution (in our general context, we will never use this regularity later). This leads to the estimate

$$
\begin{aligned}
\|D u\|_{L^{m^{*}}(\Omega)} & \leq C_{1}\|u\|_{W^{2, m}(\Omega)} \leq C_{2}\|\Delta u\|_{L^{m}(\Omega)} \\
& \leq C_{2}\left[\gamma\left\||D u|^{q}\right\|_{L^{m}(\Omega)}+\|f\|_{L^{m}(\Omega)}\right] \\
& =C_{2}\left[\gamma\|D u\|_{L^{q m}(\Omega)}^{q}+\|f\|_{L^{m}(\Omega)}\right] .
\end{aligned}
$$

For $p=2$, the necessary condition (1.3) is nothing but $q m \leq m^{*}$, hence we end up with

$$
\|D u\|_{L^{m^{*}}(\Omega)} \leq C_{3}\left[\gamma\|D u\|_{L^{m^{*}(\Omega)}}^{q}+\|f\|_{L^{m}(\Omega)}\right],
$$

but unfortunately, since $q>1$, this inequality does not provide any a priori bound for $\|D u\|_{L^{m^{*}}(\Omega)}$.

Notice also another consequence of the superlinear character $q>1$, namely that a size condition on $\|f\|_{L^{m}(\Omega)}$ is needed in order for (1.9) to be not trivial. Indeed, since (1.9) is equivalent to $\hat{F}\left(\mid D u \|_{L^{m^{*}}(\Omega)}\right) \leq\|f\|_{L^{m}(\Omega)}$, where $\hat{F}(t)=$ $\frac{1}{C_{3}} t-\gamma t^{q}$, we need that $\|f\|_{L^{m}(\Omega)}<\max _{(0, \infty)} \hat{F}(t)$ for (1.9) to be significant. As mentioned before, the necessity of a size condition is a natural feature of the problem (1.2) when $\alpha_{0}=0[1,30]$.

In order to overcome the difficulties to derive an estimate from (1.9), our idea is the following. First of all (this is a natural idea for general operators with measurable coefficients and especially for nonlinear ones, since then Calderon-Zygmund theory is not available), instead of working with $\|D u\|_{L^{m^{*}}(\Omega)}$ we will use energy estimates on $|u|^{\sigma}$ as in (1.6). In that context, in place of (1.9) we will be able to prove an estimate of the form

$$
\left\||u|^{\sigma}\right\|_{H_{0}^{1}(\Omega)}^{\frac{1}{\sigma}} \leq C_{4}\left[\gamma\left(\left\||u|^{\sigma}\right\|_{H_{0}^{1}(\Omega)}^{\frac{1}{\sigma}}\right)^{q}+\|f\|_{L^{m}(\Omega)}\right] .
$$

A crucial observation now is that the estimate (1.10) remains true even for nonnegative subsolutions of the equation. This allows us to obtain the estimate (1.10) for the family of functions $G_{k}(u)=(|u|-k)^{+}$, where $k>0$, namely

$$
\left\|\left|G_{k}(u)\right|^{\sigma}\right\|_{H_{0}^{1}(\Omega)}^{\frac{1}{\sigma}} \leq C_{4}\left[\gamma\left(\left\|\left|G_{k}(u)\right|^{\sigma}\right\|_{H_{0}^{1}(\Omega)}^{\frac{1}{\sigma}}\right)^{q}+\|f\|_{L^{m}(\Omega)}\right] \quad \forall k \geq 0 .
$$


This inequality can be rewritten as

$$
F\left(Y_{k}\right) \leq\|f\|_{L^{m}(\Omega)}, \quad \text { where } Y_{k}=\left\|\left|G_{k}(u)\right|^{\sigma}\right\|_{H_{0}^{1}(\Omega)}^{\frac{1}{\sigma}}, \quad \text { and } F(t)=\frac{1}{C_{4}} t-\gamma t^{q} .
$$

Now, if $f$ satisfies the size condition $\|f\|_{L^{m}(\Omega)}<\max _{(0, \infty)} F$, the equation $F(Y)=$ $\|f\|_{L^{m}(\Omega)}$ has two positive solutions $Y_{0}^{-}<Y_{0}^{+}$, and the inequality implies that either $Y_{k} \leq Y_{0}^{-}$or $Y_{k} \geq Y_{0}^{+}$. However, since $|u|^{\sigma} \in H_{0}^{1}(\Omega)$ (i.e. since $u$ belongs to the class (1.6)), when $k$ is large we have $Y_{k}=\left\|\left|G_{k}(u)\right|^{\sigma}\right\|_{H_{0}^{1}(\Omega)}^{\frac{1}{\sigma}}$ close to 0 and therefore $Y_{k} \leq Y_{0}^{-}$for $k$ large. By continuity, we get that $Y_{k} \leq Y_{0}^{-}$for every $k \geq 0$. This gives the desired estimate $\left\||u|^{\sigma}\right\|_{H_{0}^{1}(\Omega)} \leq Y_{0}^{-}$.

In the case where $\alpha_{0}>0$, we use the same method with a slight refinement, which allows us to remove the size condition on the data. Here the idea is that, replacing $f$ by $f-\alpha_{0} u$ and localizing on the set where $u>k$, we have

$$
\begin{aligned}
\left(f-\alpha_{0} u\right) \chi_{\{u>k\}} & \leq\left(f-\alpha_{0} u\right) \chi_{\{u>k\}} \chi_{\left\{f>\alpha_{0} u\right\}} \\
& \leq\left(f-\alpha_{0} u\right) \chi_{\left\{f>\alpha_{0} k\right\}} \chi_{\{u>k\}} \\
& \leq f \chi_{\left\{f>\alpha_{0} k\right\} .}
\end{aligned}
$$

Hence $G_{k}(u)$ will be a subsolution for a problem with a right-hand side which is suitably small as soon as $k$ is large enough. A similar difference between the case $\alpha_{0}=0$ (no zero-th order term, size condition required on the data) and the case $\alpha_{0}>0$ (no size condition required on the data) already appeared for the case of the natural growth $q=p$ studied in [21] and [25,26]. Note also that in these papers the class (1.6) is replaced by the requirement that $(\exp (\delta|u|)-1) \in W_{0}^{1, p}(\Omega)$ for some $\delta>0$ : consistently, the exponent $\sigma$ in (1.6) tends to infinity as $q$ tends to $p$.

The method described above will be used throughout the whole paper, although it will be slightly modified according to the different ranges of the growth $q$ (as explained in the next subsection). In particular, the cases when the solutions do not have finite energy, corresponding to the case $q<p-1+\frac{p}{N}$, will need some more technicalities and a few additional arguments.

\subsection{The full range of growth. Plan of the paper}

Theorems 1.1, 1.2 and 1.4, which have been stated above for the model problem (1.2), are the prototype results that we prove in this paper for the more general class of problems (1.1).

In Section 2 we give the precise assumptions satisfied by (1.1). These assumptions include in particular the case where the equation contains source terms in the form $\operatorname{div}(F(x))$ (allowed by (2.2) below) as well as transport terms of the type $B(x) D u|D u|^{p-2}$ with $|B| \in L^{N}(\Omega)$ (allowed by (2.6)-(2.7) below, as a consequence of the Young inequality).

In Section 3 we state and prove the analogue of Theorems 1.1-1.4 in this more general framework, still in the range of growth $p-1+\frac{p}{N} \leq q<p$. Here the 
solutions have finite energy (i.e. they are usual weak solutions) and satisfy the regularity (1.6) (note that $\sigma \geq 1$ in this range).

The purpose of Sections 4 and 5 is to extend these results to the range ${ }^{2} p-1<$ $q<p-1+\frac{p}{N}$. In this range we still take data $f$ in $L^{m}(\Omega)$ with the optimal exponent $m=\frac{N(q-(p-1))}{q}$; however in this range we have $m<\left(p^{*}\right)^{\prime}$, so that $f$ does not necessarily belong to $W^{-1, p^{\prime}}(\Omega)$. This implies that solutions do not in general belong to the energy space $W_{0}^{1, p}(\Omega)$ and that a generalized formulation of (1.2) is needed. We will use the notion of renormalized solution to give a meaning to (1.1) in this case.

Moreover, in this range another critical exponent naturally appears, which is $q=\frac{N(p-1)}{N-1}$. Indeed, the exponent $m$ given by the equality in (1.3) satisfies $m \geq 1$ if and only if $q \geq \frac{N(p-1)}{N-1}$. This is the reason why we will distinguish between the two cases $\frac{N(p-1)}{N-1} \leq q<p-1+\frac{p}{N}$ (Section 4) and $p-1<q<\frac{N(p-1)}{N-1}$ (Section $5)$.

In Section 4 we consider the growth $\frac{N(p-1)}{N-1}<q<p-1+\frac{p}{N}$, so that the optimal exponent $m$ varies in the interval $1<m<\left(p^{*}\right)^{\prime}$, and we prove a priori estimates, existence of solutions and bootstrap regularity for renormalized solutions which, as in Section 1.1, satisfy condition (1.6), rephrased now (since $\sigma<1$ in this range) as

$$
(1+|u|)^{\sigma-1} u \in W_{0}^{1, p}(\Omega), \quad \sigma=\frac{(N-p)(q-(p-1))}{p(p-q)} .
$$

The borderline value $q=\frac{N(p-1)}{N-1}$ corresponds to $m=1$; however, one is not allowed in this case to take general data $f \in L^{1}(\Omega)$ as we point out in Example 4.10 below. The optimal space in this case is possibly some Orlicz space (see Remark 4.12 below), but our results are only given for $f \in L^{m}(\Omega)$ with any $m>1$.

In Section 5 we deal with the range $p-1<q<\frac{N(p-1)}{N-1}$ and we take $f$ in $L^{1}(\Omega)$ or more generally in the space of bounded Radon measures, and we obtain similar results for (standard) renormalized solutions. Actually, when $\alpha_{0}>0$ (in the model problem (1.2)) the case where $f$ is a measure which charges a set of zero capacity is delicate since our method relies on some equi-integrability arguments. In this case we give two types of results: the first one concerns the case where the singular measure is small, and the second one for any singular measure but only for $p \geq 2$.

The differences in the range of exponents of $q$ and $m$, which distinguish Sections 3, 4 and 5, are summarized by the relations (2.7) and (2.8) below.

A few more words will be said in Section 7 concerning the case of Neumann or Robin boundary condition and the case where $f$ belongs to some Lorentz space (and no more to a Lebesgue space).

${ }^{2}$ As said at the beginning of the Introduction, the case $q \leq p-1$ is standard and already wellknown. 

follows:

To conclude, in view of the above comments, the paper will be organized as

2. Assumptions and notation;

3. The case $p-1+\frac{p}{N} \leq q<p$;

4. The case $\frac{N(p-1)}{N-1} \leq q<p-1+\frac{p}{N}$;

5. The case $q<\frac{N(p-1)}{N-1}$;

6 . The case $p \geq N$;

7. Further remarks.

ACKNOWLEDGEMENTS. The third author wishes to thank the Laboratoire JacquesLouis Lions de l'Université Pierre et Marie Curie for its invitation and the warm hospitality in July 2008 , when this work was mostly completed.

\section{Assumptions and notation}

Let $\Omega$ be a bounded domain in $\mathbb{R}^{N}, N \geq 1$, and let $p$ be given with $1<p<\infty$. We consider the following non linear elliptic equation:

$$
\begin{cases}-\operatorname{div}(a(x, u, D u))+a_{0}(x, u)=H(x, u, D u) & \text { in } \Omega, \\ u=0 & \text { on } \partial \Omega,\end{cases}
$$

where $a: \Omega \times \mathbb{R} \times \mathbb{R}^{N} \rightarrow \mathbb{R}^{N}, a_{0}: \Omega \times \mathbb{R} \rightarrow \mathbb{R}$ and $H: \Omega \times \mathbb{R} \times \mathbb{R}^{N} \rightarrow \mathbb{R}$ are Carathéodory functions (i.e. measurable with respect to $x$, continuous with respect to $(s, \xi))$ which satisfy, for almost every $x \in \Omega$, for every $s \in \mathbb{R}$ and for every $\xi, \xi^{\prime} \in \mathbb{R}^{N}$ the following assumptions:

$$
\begin{aligned}
& \left\{\begin{array}{l}
a(x, s, \xi) \xi \geq \alpha|\xi|^{p}-g(x), \\
\alpha>0, g \in L^{r}(\Omega), r \geq 1,
\end{array}\right. \\
& \left\{\begin{array}{l}
|a(x, s, \xi)| \leq \beta\left[|\xi|^{p-1}+|s|^{p-1}+\eta(x)\right], \\
\beta>0, \eta(x) \in L^{p-1}(\Omega),
\end{array}\right. \\
& \left(\begin{array}{l}
\left.a(x, s, \xi)-a\left(x, s, \xi^{\prime}\right)\right)\left(\xi-\xi^{\prime}\right)>0 \text { if } \xi \neq \xi^{\prime} \\
\alpha_{0} \geq 0, h \in C\left(\mathbb{R}^{+}, \mathbb{R}^{+}\right) \text {nondecreasing with } \lim _{s \rightarrow+\infty} h(s)=+\infty,
\end{array}\right. \\
& \left\{\begin{array}{l}
H(x, s, \xi) \operatorname{sign}(s) \leq \gamma|\xi|^{q}+f(x) \\
\gamma>0, p-1<q<p, \quad f \in L^{m}(\Omega), \quad m \geq 1 .
\end{array}\right.
\end{aligned}
$$


Observe that latest assumption allows $H(x, u, D u)$ to be the sum of two terms $H_{1}(x, u, D u)$ and $H_{2}(x, u, D u)$ where $H_{2}$ has a good sign (this means that $\left.H_{2}(x, u, D u) \operatorname{sign}(u) \leq 0\right)$ and $H_{1}$ has $q$-growth. Indeed, as far as a priori estimates are concerned, the difficult part lies only on the part of $H(x, u, D u)$ which has a bad sign. On the other hand, as far as the existence of solutions is concerned, a stronger hypothesis will be needed in order to control the growth of $|H(x, u, D u)|$, see (3.35) below. Similarly, assumption (2.5) is what is needed for a priori estimates, whereas some further growth condition (with respect to $x$ ) will be assumed to obtain the existence of solutions, see (3.34) below.

As said in the introduction, the exponent $m$ appearing in (2.6) must satisfy condition (1.3). A similar bound is necessary as far as the exponent $r$ appearing in (2.2) is concerned, which reads as $r \geq \frac{N(q-(p-1))}{p}$. Of course both exponents are required to be larger than one, so we will always assume that

$$
m=\max \left(\frac{N(q-(p-1))}{q}, 1\right), \quad r=\max \left(1, \frac{N(q-(p-1))}{p}\right) .
$$

As a consequence of (2.7), for $1<p<N$ we will distinguish between the following three situations depending on the $q$-growth (2.6) of the Hamiltonian $H(x, u, D u): p-1+\frac{p}{N} \leq q<p, \frac{N(p-1)}{N-1} \leq q<p-1+\frac{p}{N}$ and $q<\frac{N(p-1)}{N-1}$ and we observe that in view of (2.7):

$$
\left\{\begin{array}{l}
p-1+\frac{p}{N} \leq q<p \Longleftrightarrow\left(p^{*}\right)^{\prime} \leq m<\frac{N}{p}, 1 \leq r<\frac{N}{p}, \\
\frac{N(p-1)}{N-1}<q<p-1+\frac{p}{N} \Longleftrightarrow 1<m<\left(p^{*}\right)^{\prime}, r=1, \\
q<\frac{N(p-1)}{N-1} \Rightarrow m=1, r=1,
\end{array}\right.
$$

where, for any $s>1$, we denote by $s^{\prime}=\frac{s}{s-1}$ the conjugate exponent of $s$, and, if $1 \leq s<N$, by $s^{*}=\frac{N s}{N-s}$ the Sobolev embedding exponent.

Finally, we often use the truncation function at level $k$ defined as follows:

$$
T_{k}(s)=\max (-k, \min (s, k)) \quad \text { for every } s \in \mathbb{R},
$$

and we set

$$
G_{k}(s)=s-T_{k}(s)=(|s|-k)^{+} \operatorname{sign}(s) .
$$

\section{The case $p-1+\frac{p}{N} \leq q<p$}

In this section we consider the range of $q$ (and, correspondingly, the range of $m$ ) in the growth of the Hamiltonian (2.6) which leads to solutions of (2.1) belonging to $W_{0}^{1, p}(\Omega)$. Indeed, when $p-1+\frac{p}{N} \leq q$ we have, from (2.6)-(2.8), that $f \in L^{m}(\Omega)$ with $m=\frac{N(q-(p-1))}{q} \geq\left(p^{*}\right)^{\prime}$, hence data belong to $W^{-1, p^{\prime}}(\Omega)$. 
In this case we consider solutions of (2.1) in the usual weak sense:

$$
\left\{\begin{array}{l}
u \in W_{0}^{1, p}(\Omega), \quad a_{0}(x, u) \in L^{1}(\Omega), \quad H(x, u, D u) \in L^{1}(\Omega), \\
\int_{\Omega} a(x, u, D u) D \varphi d x+\int_{\Omega} a_{0}(x, u) \varphi d x=\int_{\Omega} H(x, u, D u) \varphi d x \\
\quad \text { for every } \varphi \in W_{0}^{1, p}(\Omega) \cap L^{\infty}(\Omega) .
\end{array}\right.
$$

Note that in (3.1) we asked that $H(x, u, D u)$ belongs to $L^{1}(\Omega)$ because the formulation of the growth condition (2.6) does not prescribe any bound from below on the growth of $H(x, u, D u) \operatorname{sign}(u)$. The same reason explains the requirement on the lower order term $a_{0}(x, u)$.

Our main result is the following a priori estimate.

Theorem 3.1. Assume (2.2), (2.3), (2.5), (2.6), (2.7), with $p-1+\frac{p}{N} \leq q<p$ (hence (2.7) reads as $\left.m=\frac{N(q-(p-1))}{q}, r=\frac{N(q-(p-1))}{p}\right)$. Assume further that either (i) $\alpha_{0}>0$

or

(ii) $\alpha_{0}=0$ and

$$
\alpha^{-\frac{p^{*}}{p m^{\prime}-p^{*}}}\|f\|_{L^{m}(\Omega)}^{\frac{p m^{\prime}}{p m^{\prime}-p^{*}}}+\alpha^{-\frac{p^{*}}{p r^{\prime}-p^{*}}}\|g\|_{L^{r}(\Omega)}^{\frac{p r^{\prime}}{p r^{\prime}}}<C^{*} \frac{\alpha^{\frac{N-q}{p-q}}}{\gamma^{\frac{N-p}{p-q}}},
$$

where $C^{*}$ is the constant depending only on $p, N$, q defined in formula (3.20) below.

Let $u$ satisfy (3.1) and the regularity condition

$$
|u|^{\sigma} \in W_{0}^{1, p}(\Omega), \quad \sigma=\frac{(N-p)(q-(p-1))}{p(p-q)} .
$$

Then we have

$$
\|u\|_{W_{0}^{1, p}(\Omega)}+\left\||u|^{\sigma}\right\|_{W_{0}^{1, p}(\Omega)} \leq M,
$$

where $M$ depends on $p, q, N, \alpha_{0}, \alpha, \gamma,|\Omega|$ and the data $f$ and $g$. When $\alpha_{0}=0$, the constant $M$ does not depend on $|\Omega|$; it depends on $f$ and $g$ only through $\|f\|_{L^{m}(\Omega)}$ and $\|g\|_{L^{r}(\Omega)}$ (in particular through the gap in (3.2)). When $\alpha_{0}>0$, the constant $M$ does not depend only on $\|f\|_{L^{m}(\Omega)}$ and $\|g\|_{L^{r}(\Omega)}$ but remains bounded when $f$ and $g$ vary in sets which are bounded and equi-integrable, respectively, in $L^{m}(\Omega)$ and in $L^{r}(\Omega)$.

Let us recall that, if $\alpha_{0}=0$, some size condition of the type of (3.2) is necessary (see $[1,30])$.

In the range of values $p-1+\frac{p}{N} \leq q<p$ we have $1 \leq \sigma<\infty$. Using Sobolev embedding theorem and the values of $\sigma$ and $m$ (which imply that $\sigma p^{*}=$ $\left.\left(m^{*}(p-1)\right)^{*}\right)$, the regularity (3.3) implies that $u \in L^{\left(m^{*}(p-1)\right)^{*}}(\Omega)$. 
Remark 3.2. Assumption (2.3) will not be used in the proof below but for ensuring that the term $a(x, u, D u)$ belongs to $L^{p^{\prime}}(\Omega)^{N}$ whenever $u \in W_{0}^{1, p}(\Omega)$. Therefore Theorem 3.1 remains true if one removes assumption (2.3) and consider solutions $u$ such that $a(x, u, D u) \in L^{p^{\prime}}(\Omega)^{N}$.

Remark 3.3. The case where assumption (2.5) is relaxed into

$$
\left\{\begin{array}{l}
\exists L>0: \quad a(x, s) \operatorname{sign}(s) \geq \alpha_{0} h(|s|) \quad \forall|s| \geq L, \\
\alpha_{0} \geq 0, h \in C\left(\mathbb{R}^{+}, \mathbb{R}^{+}\right) \text {nondecreasing with } \lim _{s \rightarrow+\infty} h(s)=+\infty,
\end{array}\right.
$$

can be reduced to the previous one, by defining $\tilde{a}_{0}(x, s)=\left(a_{0}(x, s) \operatorname{sign}(s)\right)^{+} \operatorname{sign}(s)$ and replacing $f$ with $\tilde{f}=f+\left|a_{0}(x, s)-\tilde{a}_{0}(x, s)\right|$. Assuming, for instance, that $\sup _{|s| \leq L} a_{0}(x, s)$ belongs to $L^{\infty}(\Omega)$ makes this correction harmless, since we only add a bounded term to $f$. In this case, nothing changes if $\alpha_{0}>0$, while if $\alpha_{0}=0$ the condition (3.2) has to be modified replacing $f$ with $\tilde{f}$.

Remark 3.4. The conclusion of Theorem 3.1 remains true for nonnegative subsolutions of the problem

$$
\left\{\begin{array}{l}
-\operatorname{div}(a(x, z, D z))+\alpha_{0} h(z)=\gamma|D z|^{q}+f \text { in } \Omega, \\
z=0 \text { on } \partial \Omega
\end{array}\right.
$$

namely for $z$ satisfying

$$
\left\{\begin{array}{l}
z \in W_{0}^{1, p}(\Omega), z \geq 0, z^{\sigma} \in W_{0}^{1, p}(\Omega) \text { with } \sigma=\frac{(N-p)(q-(p-1))}{p(p-q)}, h(z) \in L^{1}(\Omega), \\
\int_{\Omega} a(x, z, D z) D \varphi d x+\alpha_{0} \int_{\Omega} h(z) \varphi d x \leq \gamma \int_{\Omega}|D z|^{q} \varphi d x+\int_{\Omega} f(x) \varphi d x \\
\quad \text { for every } \varphi \in W_{0}^{1, p}(\Omega) \cap L^{\infty}(\Omega), \varphi \geq 0 .
\end{array}\right.
$$

Indeed, we will see that using assumptions (2.5) and (2.6), the proof of the a priori estimate reduces to this case, starting from (3.8) below (or from (3.28) when $q=$ $\left.p-1+\frac{p}{N}\right)$.

Let us stress that in some situations it is interesting to use the result for subsolutions. In particular, this allows one to separate estimates on $u^{+}$and $u^{-}$, whenever $u$ is a solution. As an example, consider the model problem (1.2), i.e.

$$
\begin{cases}-\Delta_{p}(u)+\alpha_{0} u=\gamma|D u|^{q}+f(x) & \text { in } \Omega \\ u=0 & \text { on } \partial \Omega .\end{cases}
$$

It is well-known that if $u \in W_{0}^{1, p}(\Omega)$ is a solution of (3.5), then $u^{+}$satisfies

$$
-\Delta_{p}\left(u^{+}\right)+\alpha_{0} u^{+} \leq \gamma\left|D u^{+}\right|^{q}+f(x) \chi_{u \geq 0},
$$

while $u^{-}$satisfies

$$
-\Delta_{p}\left(u^{-}\right)+\alpha_{0} u^{-} \leq-\gamma\left|D u^{-}\right|^{q}-f(x) \chi_{u \leq 0} .
$$


Now, in the equation for $u^{-}$, the Hamiltonian has a good sign and in particular $u^{-}$ satisfies

$$
-\Delta_{p}\left(u^{-}\right)+\alpha_{0} u^{-} \leq-f(x) \chi_{u \leq 0} .
$$

Therefore an estimate for $u^{-}$follows from standard results, without any extra condition (in particular, no size condition at all). On the other hand, $u^{+}$satisfies

$$
-\Delta_{p}\left(u^{+}\right)+\alpha_{0} u^{+} \leq \gamma\left|D u^{+}\right|^{q}+f^{+}(x) \chi_{u \geq 0}
$$

and an estimate for $u^{+}$follows by applying the present variant of Theorem 3.1 for subsolutions, with $f^{+}$as source term. In that case, one concludes that for problem (3.5) the necessary conditions (in particular, the size condition when $\alpha_{0}=0$ ) only concern $f^{+}$.

In the proof of Theorem 3.1 we need to distinguish the case $p-1+\frac{p}{N}<q<p$ from the limiting value $q=p-1+\frac{p}{N}$.

Proof of Theorem 3.1 in the case $p-1+\frac{p}{N}<q<p$. Let $G_{k}(s)$ be defined from (2.9). We wish to take $\left|G_{k}(u)\right|^{\lambda-1} G_{k}(u)$ as test function in (3.1), where

$$
\lambda=\frac{(p-1) N(m-1)}{N-m p}=\frac{N(q-(p-1))-q}{(p-q)} .
$$

Observe that since $p-1+\frac{p}{N}<q<p$ we have $1<\lambda<+\infty$. However, this test function is not allowed, a priori, in (3.1): so we actually multiply by $\left|T_{n}\left(G_{k}(u)\right)\right|^{\lambda-1} T_{n}\left(G_{k}(u)\right)$ and then we let $n$ tend to infinity. To this purpose, we first apply (2.2), (2.5) and (2.6) to obtain

$$
\begin{aligned}
& \alpha \lambda \int_{\Omega}\left|D T_{n}(u)\right|^{p}\left|T_{n}\left(G_{k}(u)\right)\right|^{\lambda-1} d x+\alpha_{0} \int_{\Omega} h(|u|)\left|T_{n}\left(G_{k}(u)\right)\right|^{\lambda} d x \\
& \leq \gamma \int_{\Omega}|D u|^{q}\left|T_{n}\left(G_{k}(u)\right)\right|^{\lambda} d x+\int_{\Omega}|f|\left|T_{n}\left(G_{k}(u)\right)\right|^{\lambda} d x \\
& \quad+\lambda \int_{\Omega}|g|\left|T_{n}\left(G_{k}(u)\right)\right|^{\lambda-1} d x,
\end{aligned}
$$

and then we let $n$ tend to infinity, using Fatou's lemma in the left-hand side and Lebesgue theorem in the right-hand side. Indeed, since $u$ belongs to (3.3), we have $|u|^{\sigma-1}|D u| \in L^{2}(\Omega)$ and $|u|^{\sigma} \in L^{2^{*}}(\Omega)$; hence a standard application of Hölder inequality ensures that $|D u|^{q}\left|G_{k}(u)\right|^{\lambda} \in L^{1}(\Omega)$, and since $f \in L^{m}(\Omega)$ and $g \in L^{r}(\Omega)$ with the values of $m$ and $r$ given by (2.7), we have $f\left|G_{k}(u)\right|^{\lambda}$ and $g\left|G_{k}(u)\right|^{\lambda-1} \in L^{1}(\Omega)$ as well. Therefore we obtain

$$
\begin{aligned}
& \alpha \lambda \int_{\Omega}|D u|^{p}\left|G_{k}(u)\right|^{\lambda-1} d x+\alpha_{0} \int_{\Omega} h(|u|)\left|G_{k}(u)\right|^{\lambda} d x \\
& \leq \gamma \int_{\Omega}|D u|^{q}\left|G_{k}(u)\right|^{\lambda} d x+\int_{\Omega}|f|\left|G_{k}(u)\right|^{\lambda} d x \\
& \quad+\lambda \int_{\Omega}|g|\left|G_{k}(u)\right|^{\lambda-1} d x .
\end{aligned}
$$


We have

$$
\alpha \lambda \int_{\Omega}|D u|^{p}\left|G_{k}(u)\right|^{\lambda-1} d x=\alpha C \int_{\Omega}\left|D\left(\left|G_{k}(u)\right|^{\sigma}\right)\right|^{p} d x,
$$

where

$$
\sigma=\frac{\lambda-1}{p}+1=\frac{(N-p)(q-(p-1))}{p(p-q)},
$$

and $C$ denote constants only depending on $p, N, q$ (recall that $\lambda$ is also defined in terms of $p, N, q)$.

Similarly, by Hölder's inequality and the definition of $\sigma$

$$
\begin{aligned}
\gamma \int_{\Omega}|D u|^{q} G_{k}(u)^{\lambda} d x & \leq \gamma\left(\int_{\Omega}|D u|^{p} G_{k}(u)^{\lambda-1} d x\right)^{\frac{q}{p}}\left(\int_{\Omega}\left|G_{k}(u)\right|^{\lambda+\frac{q}{p-q}} d x\right)^{1-\frac{q}{p}} \\
& =\gamma C\left(\int_{\Omega}\left|D\left(\left|G_{k}(u)\right|^{\sigma}\right)\right|^{p} d x\right)^{\frac{q}{p}}\left(\int_{\Omega}\left|G_{k}(u)\right|^{\lambda+\frac{q}{p-q}} d x\right)^{1-\frac{q}{p}} .
\end{aligned}
$$

But the definition of $\lambda$ and $\sigma$ imply that

$$
\lambda+\frac{q}{p-q}=\sigma p^{*},
$$

hence by Sobolev's embedding theorem we obtain

$$
\gamma \int_{\Omega}|D u|^{q} G_{k}(u)^{\lambda} d x \leq \gamma C\left(\int_{\Omega}\left|D\left(\left|G_{k}(u)\right|^{\sigma}\right)\right|^{p} d x\right)^{\frac{q}{p}+\left(1-\frac{q}{p}\right) \frac{p^{*}}{p}} .
$$

Concerning the second term of the right-hand side of (3.8) we have

$$
\begin{aligned}
\int_{\Omega}|f|\left|G_{k}(u)\right|^{\lambda} d x & =\int_{\left\{|f| \leq \frac{\alpha_{0}}{2} h(|u|)\right\}}|f|\left|G_{k}(u)\right|^{\lambda} d x+\int_{\left\{|f|>\frac{\alpha_{0}}{2} h(|u|)\right\}}|f|\left|G_{k}(u)\right|^{\lambda} d x \\
& \leq \frac{\alpha_{0}}{2} \int_{\Omega} h(|u|)\left|G_{k}(u)\right|^{\lambda} d x+\int_{\left\{|f|>\frac{\alpha_{0}}{2} h(k)\right\}}|f|\left|G_{k}(u)\right|^{\lambda} d x .
\end{aligned}
$$

The first term of the right-hand side of (3.12) is absorbed by the second term of the left-hand side of (3.8). Using Hölder's inequality, and since

$$
\lambda m^{\prime}=\frac{(p-1) N m}{N-m p}=\sigma p^{*},
$$

we estimate the second term by

$$
\begin{aligned}
\int_{\left\{|f|>\frac{\alpha_{0}}{2} h(k)\right\}}\left|f \| G_{k}(u)\right|^{\lambda} d x & \leq\left\|f \chi_{\left\{|f|>\frac{\alpha_{0}}{2} h(k)\right\}}\right\|_{L^{m}(\Omega)}\left\|\left|G_{k}(u)\right|^{\lambda}\right\|_{L^{m^{\prime}}(\Omega)} \\
& \leq C\left\|f \chi_{\left\{|f|>\frac{\alpha_{0}}{2} h(k)\right\}}\right\|_{L^{m}(\Omega)}\left\|D\left(\left|G_{k}(u)\right|^{\sigma}\right)\right\|_{L^{p}(\Omega)}^{{\frac{p^{\prime}}{m^{\prime}}}^{m^{\prime}}} .
\end{aligned}
$$


Similarly, we have

$$
\begin{aligned}
& \int_{\Omega}|g|\left|G_{k}(u)\right|^{\lambda-1} d x \\
= & \left.\left.\int_{\left\{|g| \leq \frac{\alpha_{0}}{2}\right.} h(|u|)\left|G_{k}(u)\right|\right\}\left|G_{k}(u)\right|^{\lambda-1} d x+\int_{\left\{|g|>\frac{\alpha_{0}}{2}\right.} h(|u|)\left|G_{k}(u)\right|\right\} \\
\leq & \frac{\alpha_{0}}{2} \int_{\Omega} h\left(|g|\left|G_{k}(u)\right|^{\lambda-1} d x\right. \\
\left.\left.\int_{\left\{|g|>\frac{\alpha_{0}}{2}\right.} h(|u|)\left|G_{k}(u)\right|^{\lambda} d x\right) \mid\right\} & |g|\left|G_{k}(u)\right|^{\lambda-1} d x .
\end{aligned}
$$

The latest term can be furtherly split as

$$
\begin{aligned}
\int_{\left\{|g|>\frac{\alpha_{0}}{2} h(|u|)\left|G_{k}(u)\right|\right\}}|g|\left|G_{k}(u)\right|^{\lambda-1} d x= & \int_{\left\{|g|>\frac{\alpha_{0}}{2} h(|u|)\left|G_{k}(u)\right|\right\}}|g|\left|G_{k}(u)\right|^{\lambda-1} \chi_{\left\{\left|G_{k}(u)\right| \leq \delta_{k}\right\}} d x \\
& +\int_{\left\{|g|>\frac{\alpha_{0}}{2} h(|u|)\left|G_{k}(u)\right|\right\}}|g|\left|G_{k}(u)\right|^{\lambda-1} \chi_{\left\{\left|G_{k}(u)\right|>\delta_{k}\right\}} d x \\
\leq & \delta_{k}^{\lambda-1}\|g\|_{L^{1}(\Omega)}+\int_{\left\{|g|>\frac{\alpha_{0}}{2} h(k) \delta_{k}\right\}}|g|\left|G_{k}(u)\right|^{\lambda-1} d x .
\end{aligned}
$$

Let us choose for instance $\delta_{k}=\frac{\alpha_{0}}{\sqrt{h(k)}}$ : with this choice we emphasize that $\delta_{k}$ can be taken to be zero if $\alpha_{0}=0$, since in that case we do not need this technicality. We obtain

$$
\begin{gathered}
\int_{\Omega}|g|\left|G_{k}(u)\right|^{\lambda-1} d x \leq \frac{\alpha_{0}}{2} \int_{\Omega} h(|u|)\left|G_{k}(u)\right|^{\lambda} d x \\
+\left(\frac{\alpha_{0}}{\sqrt{h(k)}}\right)^{\lambda-1}\|g\|_{L^{1}(\Omega)}+\int_{\left\{|g|>\frac{\alpha_{0}^{2}}{2} \sqrt{h(k)}\right\}}|g|\left|G_{k}(u)\right|^{\lambda-1} d x .
\end{gathered}
$$

Moreover by Hölder inequality we get

$\int_{\left\{|g|>\frac{\alpha_{0}^{2}}{2} \sqrt{h(k)}\right\}}|g|\left|G_{k}(u)\right|^{\lambda-1} d x \leq\left\|g \chi_{\left\{|g|>\frac{\alpha_{0}^{2}}{2} \sqrt{h(k)}\right\}}\right\|_{L^{r}(\Omega)}\left(\int_{\Omega}\left|G_{k}(u)\right|^{(\lambda-1) r^{\prime}} d x\right)^{1-\frac{1}{r}}$, and since $(\lambda-1) r^{\prime}=\frac{N(p-1) m}{N-m p}=\sigma p^{*}$, Sobolev inequality implies

$\int_{\left\{|g|>\frac{\alpha_{0}^{2}}{2} \sqrt{h(k)}\right\}}|g|\left|G_{k}(u)\right|^{\lambda-1} d x \leq\left\|g \chi_{\left\{|g|>\frac{\alpha_{0}^{2}}{2} \sqrt{h(k)}\right\}}\right\| L_{L^{r}(\Omega)}\left\|D\left(\left|G_{k}(u)\right|^{\sigma}\right)\right\|_{L^{p}(\Omega)}^{\frac{p^{*}}{r^{\prime}}}$. 
Set

$$
Y_{k}=\left\|D\left(\left|G_{k}(u)\right|^{\sigma}\right)\right\|_{L^{p}(\Omega)} .
$$

From (3.8), (3.9), (3.11), (3.13), (3.14) and (3.15), we now deduce that there exist positive constants $C_{1}$ and $C_{2}$ such that

$$
\begin{aligned}
\alpha C_{1} Y_{k}^{p} \leq & \gamma C_{2} Y_{k}^{q+\left(1-\frac{q}{p}\right) p^{*}}+\left\|f \chi_{\left\{|f|>\frac{\alpha_{0}}{2} h(k)\right\}}\right\|_{L^{m}(\Omega)} Y_{k}^{\frac{p^{*}}{m^{\prime}}} \\
& +\left(\frac{\alpha_{0}}{\sqrt{h(k)}}\right)^{\lambda-1}\|g\|_{L^{1}(\Omega)}+\left\|g \chi_{\left\{|g|>\frac{\alpha_{0}^{2}}{2} \sqrt{h(k)}\right\}}\right\|_{L^{r}(\Omega)} Y_{k}^{\frac{p^{*}}{r^{\prime}}}
\end{aligned}
$$

Then, using that $\frac{p^{*}}{m^{\prime}}$ and $\frac{p^{*}}{r^{\prime}}$ are both less than $p$, Young's inequality implies

$$
\begin{aligned}
\frac{\alpha C_{1}}{2} Y_{k}^{p} \leq & \gamma C_{2} Y_{k}^{q+\left(1-\frac{q}{p}\right) p^{*}}+C_{3} \alpha^{-\frac{p^{*}}{p m^{\prime}-p^{*}}}\left\|f \chi_{\left\{|f|>\frac{\alpha_{0}}{2} h(k)\right\}}\right\|_{L^{m}(\Omega)}^{\frac{p m^{\prime}}{p m^{\prime}-p^{*}}} \\
& +\left(\frac{\alpha_{0}}{\sqrt{h(k)}}\right)^{\lambda-1}\|g\|_{L^{1}(\Omega)}+C_{4} \alpha^{-\frac{p^{*}}{p r^{\prime}-p^{*}}}\left\|g \chi_{\left\{|g|>\frac{\alpha_{0}^{2}}{2} \sqrt{h(k)\}}\right.}\right\|_{L^{r}(\Omega)}^{\frac{p r^{\prime}}{p r^{\prime}-p^{*}}} .
\end{aligned}
$$

Thus we finally obtain, for positive constants $C_{5}, C_{6}$ :

$$
\begin{aligned}
\forall k \geq 0, \quad C_{5} \alpha Y_{k}^{p}-\gamma C_{6} Y_{k}^{p+\left(1-\frac{q}{p}\right)\left(p^{*}-p\right)} \leq \alpha^{-\frac{p^{*}}{p m^{\prime}-p^{*}}}\left\|f \chi_{\left\{|f|>\frac{\alpha_{0}}{2} h(k)\right\}}\right\|_{L^{m}(\Omega)}^{\frac{p m^{\prime}}{p m^{\prime}-p^{*}}} \\
+\left(\frac{\alpha_{0}}{\sqrt{h(k)}}\right)^{\lambda-1}\|g\|_{L^{1}(\Omega)}+\alpha^{-\frac{p^{*}}{p r^{\prime}-p^{*}}\left\|g \chi_{\left\{|g|>\frac{\alpha_{0}^{2}}{2} \sqrt{h(k)}\right\}}\right\|_{L^{r}(\Omega)}^{\frac{p r^{\prime}}{p r^{\prime} p^{*}}}}
\end{aligned}
$$

Define the function $F: \mathbb{R}^{+} \rightarrow \mathbb{R}$ by

$$
F(Y)=C_{5} \alpha Y^{p}-\gamma C_{6} Y^{p+\left(1-\frac{q}{p}\right)\left(p^{*}-p\right)} .
$$

Then (3.16) is equivalent to

$$
\forall k \geq 0, \quad F\left(Y_{k}\right) \leq M_{k},
$$

where

$$
\begin{aligned}
M_{k}:= & \alpha^{-\frac{p^{*}}{p m^{\prime}-p^{*}}}\left\|f \chi_{\left\{|f|>\frac{\alpha_{0}}{2} h(k)\right\}}\right\|_{L^{m}(\Omega)}^{\frac{p m^{\prime}}{p m^{\prime}-p^{*}}}+\left(\frac{\alpha_{0}}{\sqrt{h(k)}}\right)^{\lambda-1}\|g\|_{L^{1}(\Omega)} \\
& +\alpha^{-\frac{p^{*}}{p r^{\prime}-p^{*}}}\left\|g \chi_{\left\{|g|>\frac{\alpha_{0}^{2}}{2} \sqrt{h(k)}\right\}}\right\|_{L^{r}(\Omega)}^{\frac{p r^{\prime}}{p r^{\prime}-p^{*}}} .
\end{aligned}
$$

Since $q<p, F$ is a concave function with a unique maximizer $Z^{*}=C_{7}\left(\frac{\alpha}{\gamma}\right)^{\frac{N}{p^{*}(p-q)}}$ and maximum value

$$
F^{*}=F\left(Z^{*}\right)=C^{*} \alpha^{\frac{N-q}{p-q}} \gamma^{-\frac{N-p}{p-q}}
$$


Inequality (3.18) is non trivial only if its right-hand side is strictly smaller than $F^{*}$. Indeed, for any $M$ such that $0 \leq M<F^{*}$, the equation

$$
F(Y)=M
$$

has two roots that we denote by $Z^{-}(M)$ and $Z^{+}(M)$, with

$$
0 \leq Z^{-}(M)<Z^{*}<Z^{+}(M) \text {. }
$$

Here we split the proof into two cases.

(i) If $\alpha_{0}=0$, note that $M_{k}$ defined in (3.19) does not depend on $k$ and inequality (3.18) becomes $F\left(Y_{k}\right) \leq M_{0}$ where

$$
M_{0}=\alpha^{-\frac{p^{*}}{p m^{\prime}-p^{*}}}\|f\|_{L^{m}(\Omega)}^{\frac{p m^{\prime}}{p m^{\prime}-p^{*}}}+\alpha^{-\frac{p^{*}}{p r^{\prime}-p^{*}}}\|g\|_{L^{r}(\Omega)}^{\frac{p r^{\prime}}{p r^{\prime}-p^{*}}} .
$$

Here we use assumption (3.2), which is nothing but

$$
M_{0}<F^{*}
$$

Therefore, inequality (3.18) is equivalent to

$$
\forall k \geq 0, \quad \text { either } \quad Y_{k} \leq Z^{-}\left(M_{0}\right) \quad \text { or } \quad Y_{k} \geq Z^{+}\left(M_{0}\right) \text {. }
$$

But since $|u|^{\sigma-1} u \in W_{0}^{1, p}(\Omega)$, the function $k \rightarrow Y_{k}=\left\|D\left(\left|G_{k}(u)\right|^{\sigma}\right)\right\|_{L^{p}(\Omega)}$ is continuous and tends to zero when $k$ tends to infinity. Then, by continuity, the alternative (3.22) implies that $Y_{k} \leq Z^{-}\left(M_{0}\right)$ for every $k$; in particular, one has

$$
Y_{0}=\left\|D\left(|u|^{\sigma}\right)\right\|_{L^{p}(\Omega)} \leq Z^{-}\left(M_{0}\right)<Z^{*} .
$$

(ii) If $\alpha_{0}>0$, we define $k^{*}$ as

$$
k^{*}=\inf \left\{k>0: M_{k}<F^{*}\right\}
$$

where $M_{k}$ is defined in (3.18).

Since $M_{k}$ is nonincreasing with respect to $k$, this means that for every $k>k^{*}$ we have $M_{k}<F^{*}$, hence the equation $F(Y)=M_{k}$ has two roots $Z^{-}\left(M_{k}\right)$ and $Z^{+}\left(M_{k}\right)$, with $0<Z^{-}\left(M_{k}\right)<Z^{*}<Z^{+}\left(M_{k}\right)$. Therefore inequality (3.18) implies that either $Y_{k} \leq Z^{-}\left(M_{k}\right)$ or $Y_{k} \geq Z^{+}\left(M_{k}\right)$, which means in particular that $Y_{k}$ belongs either to $\left(0, Z^{*}\right)$ or to $\left(Z^{*}, \infty\right)$ for every $k>k^{*}$. Since the function $k \rightarrow Y_{k}$ is continuous and tends to zero when $k$ tends to infinity, we deduce by continuity that $Y_{k}<Z^{*}$ for every $k>k^{*}$, and therefore we have

$$
Y_{k} \leq Z^{-}\left(M_{k}\right) \quad \forall k>k^{*} .
$$

In both cases, we deduce from either (3.23) or (3.25) that

$$
\left\|D\left(\left|G_{k^{*}}(u)\right|^{\sigma}\right)\right\|_{L^{p}(\Omega)} \leq Z^{*}
$$

where $k^{*}=0$ when $\alpha_{0}=0$, and where $k^{*}$ is defined by (3.24) when $\alpha_{0}>0$. 
Note that the constant $Z^{*}$ depends only on $N, q, \alpha$ and $\gamma$, but that $k^{*}$ depends also on the functions $f$ and $g$.

Clearly, estimate (3.26) provides an estimate of $\left\|D G_{k_{1}^{*}}(u)\right\|_{L^{p}(\Omega)}$ for $k_{1}^{*}=$ $k^{*}+1$. We then use $v=T_{k_{1}^{*}}(u)$ in (2.1) and we get

$$
\begin{aligned}
\alpha \int_{\Omega} \mid D T_{\left.k_{1}^{*}(u)\right|^{p} d x \leq} & \int_{\Omega}\left(\gamma|D u|^{q}+f\right)\left|T_{k_{1}^{*}}(u)\right| d x \\
\leq & \gamma k_{1}^{*} \int_{\Omega}\left|D T_{k_{1}^{*}}(u)\right|^{q} d x+\gamma k_{1}^{*} \int_{\Omega}\left|D G_{k_{1}^{*}}(u)\right|^{q} d x \\
& +k_{1}^{*}\|f\|_{L^{1}(\Omega)},
\end{aligned}
$$

from which we deduce, using $q<p$ and the estimate on $\left\|D G_{k_{1}^{*}}(u)\right\|_{L^{p}(\Omega)}$, an estimate on $\left\|D T_{k_{1}^{*}}(u)\right\|_{L^{p}(\Omega)}$, with a constant which depends on $k^{*}$. This yields an estimate for $\left\|D T_{k_{1}^{*}}\left(|u|^{\sigma}\right)\right\|_{L^{p}(\Omega)}$ and this concludes the proof of the estimate (3.4).

Remark 3.5. In the case $\alpha_{0}>0$, estimate (3.25), together with the definition (3.19) of $M_{k}$, implies that

$$
\lim _{k \rightarrow+\infty}\left\|D\left(\left|G_{k}(u)\right|^{\sigma}\right)\right\|_{L^{p}(\Omega)}=0
$$

uniformly when $f$ and $g$ vary in sets that are bounded and equi-integrable in $L^{m}(\Omega)$ and $L^{r}(\Omega)$, respectively.

Then, when $f$ and $g$ vary in such sets, Sobolev inequality implies that, uniformly,

$$
\lim _{k \rightarrow+\infty}\left\|G_{k}(u)\right\|_{L^{\sigma p^{*}}(\Omega)}=0
$$

which is the equi-integrability of $u$ in $L^{\sigma} p^{*}(\Omega)$.

Remark 3.6. A variant of the above proof consists in keeping from the beginning the information that all integrals are restricted where $|u|>k$. Then (3.18) is replaced by

$$
\forall k \geq 0, \quad F\left(Y_{k}\right) \leq \tilde{M}_{k}
$$

where (compare with (3.19))

$$
\begin{aligned}
\tilde{M}_{k}:= & \alpha^{-\frac{p^{*}}{p m^{\prime}-p^{*}}}\left\|f \chi_{\left\{|f|>\frac{\alpha_{0}}{2} h(k),|u|>k\right\}}\right\|_{L^{m}(\Omega)}^{\frac{p m^{\prime}}{p m^{\prime}-p^{*}}}+\left(\frac{\alpha_{0}}{\sqrt{h(k)}}\right)^{\lambda-1}\|g\|_{L^{1}(\Omega)} \\
& +\alpha^{-\frac{p^{*}}{p r^{\prime}-p^{*}}}\left\|g \chi_{\left\{|g|>\frac{\alpha_{0}^{2}}{2} \sqrt{h(k)},|u|>k\right\}}\right\|_{L^{r}(\Omega)}^{\frac{p r^{\prime}}{p r^{\prime}-p^{*}}} .
\end{aligned}
$$

In particular, if $\alpha_{0}=0$, we have

$$
\tilde{M}_{k}:=\alpha^{-\frac{p^{*}}{p m^{\prime}-p^{*}}}\left\|f \chi_{|u|>k\}}\right\|_{L^{m}(\Omega)}^{\frac{p m^{\prime}}{p m^{\prime}-p^{*}}}+\alpha^{-\frac{p^{*}}{p r^{\prime}-p^{*}}}\left\|g \chi_{\{|u|>k\}}\right\|_{L^{r}(\Omega)}^{\frac{p r^{\prime}}{p r^{\prime}-p^{*}}} .
$$


Then, if we define

$$
\tilde{k}^{*}=\inf \left\{k>0: \tilde{M}_{k}<F^{*}\right\}
$$

proceeding like in the above proof we obtain estimate (3.25) with $\tilde{M}_{k}$ instead of $M_{k}$, and for every $k>\tilde{k}^{*}$. Note that now $\tilde{k}^{*}$ depends on the level sets of $u$, the measure of which is controlled by $\|u\|_{L^{1}(\Omega)}$.

Therefore, in this variant we obtain estimate (3.4) with a constant $M$ depending also on $\|u\|_{L^{1}(\Omega)}$. This constant remains bounded when $\|u\|_{L^{1}(\Omega)}$ is bounded and when $f$ and $g$ vary in sets which are bounded and equi-integrable in $L^{m}(\Omega)$ and $L^{r}(\Omega)$ respectively.

Moreover, as in the previous Remark, we also obtain that

$$
\lim _{k \rightarrow+\infty}\left\|G_{k}(u)\right\|_{L^{\sigma p^{*}}(\Omega)}=0
$$

uniformly whenever $\|u\|_{L^{1}(\Omega)}$ is bounded and $f$ and $g$ vary in sets which are bounded and equi-integrable in $L^{m}(\Omega)$ and $L^{r}(\Omega)$, respectively.

This variant may have some interest if $\alpha_{0}=0$ when the size condition (3.21) does not hold, and we will use it in this spirit in the regularity result of Theorem 3.8.

Proof of Theorem 3.1 in the case $q=p-1+\frac{p}{N}$. This case corresponds to $f \in$ $L^{m}(\Omega), m=\left(p^{*}\right)^{\prime}, g \in L^{r}(\Omega), r=1$ and $\lambda=\sigma=1$; this means that solutions are expected to belong precisely to the energy space $W_{0}^{1, p}(\Omega)$. The above proof remains unchanged in the case $\alpha_{0}=0$. However, if $\alpha_{0}>0$, it needs to be slightly modified, because of the term $g(x)$ which appears in (2.2). In fact, in the previous situation we used $\lambda>1$ to deduce, from (3.19), that $M_{k}$ is small enough for large $k$. Since now $\lambda=1$, we refine a bit the previous proof, by slightly changing our test function near $u=k$. So, we choose now

$$
v=G_{k}(u) \frac{\left|G_{k}(u)\right|}{\varepsilon+\left|G_{k}(u)\right|}
$$

as test function, where $\varepsilon$ will be chosen later. Actually, as in the proof of the previous case, we need to start with bounded test functions in (3.1). So we take in (3.1) $\varphi=T_{n}\left(G_{k}(u)\right) \frac{\left|T_{n}\left(G_{k}(u)\right)\right|}{\varepsilon+\left|T_{n}\left(G_{k}(u)\right)\right|}$ and we use assumptions (2.2), (2.5), (2.6) to obtain

$$
\begin{aligned}
& \alpha \int_{\Omega}\left|D T_{n}(u)\right|^{p} \frac{T_{n}\left(G_{k}(u)\right)^{2}+2 \varepsilon\left|T_{n}\left(G_{k}(u)\right)\right|}{\left(\varepsilon+\left|T_{n}\left(G_{k}(u)\right)\right|\right)^{2}} d x+\alpha_{0} \int_{\Omega} h(|u|) \frac{T_{n}\left(G_{k}(u)\right)^{2}}{\varepsilon+\left|T_{n}\left(G_{k}(u)\right)\right|} d x \\
& \leq \gamma \int_{\Omega}|D u|^{q} \frac{T_{n}\left(G_{k}(u)\right)^{2}}{\varepsilon+\left|T_{n}\left(G_{k}(u)\right)\right|} d x+\int_{\Omega} f \frac{T_{n}\left(G_{k}(u)\right)^{2}}{\varepsilon+\left|T_{n}\left(G_{k}(u)\right)\right|} d x \\
& \quad+\int_{\Omega}|g| \frac{T_{n}\left(G_{k}(u)\right)^{2}+2 \varepsilon\left|T_{n}\left(G_{k}(u)\right)\right|}{\left(\varepsilon+\left|T_{n}\left(G_{k}(u)\right)\right|\right)^{2}} d x .
\end{aligned}
$$


Then we pass to the limit in $n$, and we get, after using $\frac{|t|}{\varepsilon+|t|} \leq \frac{t^{2}+2 \varepsilon|t|}{(\varepsilon+|t|)^{2}} \leq \frac{2|t|}{\varepsilon+|t|}$ :

$$
\begin{aligned}
& \alpha \int_{\Omega}|D u|^{p} \frac{\left|G_{k}(u)\right|}{\varepsilon+\left|G_{k}(u)\right|} d x+\alpha_{0} \int_{\Omega} h(|u|) \frac{G_{k}(u)^{2}}{\varepsilon+\left|G_{k}(u)\right|} d x \\
& \leq \gamma \int_{\Omega}|D u|^{q} \frac{G_{k}(u)^{2}}{\varepsilon+\left|G_{k}(u)\right|} d x+\int_{\Omega} f \frac{G_{k}(u)^{2}}{\varepsilon+\left|G_{k}(u)\right|} d x \\
& \quad+2 \int_{\Omega}|g| \frac{\left|G_{k}(u)\right|}{\varepsilon+\left|G_{k}(u)\right|} d x .
\end{aligned}
$$

We have

$$
\alpha \int_{\Omega}|D u|^{p} \frac{\left|G_{k}(u)\right|}{\varepsilon+\left|G_{k}(u)\right|} d x=\alpha \int_{\Omega} \mid D\left(\left.\varphi_{\varepsilon}\left(G_{k}(u)\right)\right|^{p} d x,\right.
$$

where

$$
\varphi_{\varepsilon}(s)=\int_{0}^{s}\left(\frac{|\xi|}{\varepsilon+|\xi|}\right)^{\frac{1}{p}} d \xi
$$

We have, using that $\frac{p}{p-q}=p^{*}$ since $q=p-1+\frac{p}{N}$,

$$
\begin{aligned}
\int_{\Omega}|D u|^{q} \frac{G_{k}(u)^{2}}{\varepsilon+\left|G_{k}(u)\right|} d x & =\int_{\Omega}\left|D \varphi_{\varepsilon}\left(G_{k}(u)\right)\right|^{q}\left|G_{k}(u)\right|\left(\frac{\left|G_{k}(u)\right|}{\varepsilon+\left|G_{k}(u)\right|}\right)^{1-\frac{q}{p}} d x \\
& \leq\left(\int_{\Omega}\left|D \varphi_{\varepsilon}\left(G_{k}(u)\right)\right|^{p} d x\right)^{\frac{q}{p}}\left(\int_{\Omega}\left|G_{k}(u)\right|^{p^{*}} d x\right)^{1-\frac{q}{p}} .
\end{aligned}
$$

Since we have $s \leq c\left(\varphi_{\varepsilon}(s)+\varepsilon\right)$, we deduce

$$
\int_{\Omega}|D u|^{q} \frac{G_{k}(u)^{2}}{\varepsilon+\left|G_{k}(u)\right|} d x \leq C\left(\int_{\Omega}\left|D \varphi_{\varepsilon}\left(G_{k}(u)\right)\right|^{p} d x\right)^{\frac{q}{p}}\left(\int_{\Omega}\left(\varphi_{\varepsilon}\left(G_{k}(u)\right)+\varepsilon\right)^{p^{*}} d x\right)^{1-\frac{q}{p}} .
$$

We deal with the term containing $f$ exactly as in (3.13), obtaining, since now $m=$ $\left(p^{*}\right)^{\prime}$,

$$
\begin{aligned}
\int_{\Omega} f \frac{G_{k}(u)^{2}}{\varepsilon+\left|G_{k}(u)\right|} d x \leq & \frac{\alpha_{0}}{2} \int_{\Omega} h(|u|) \frac{G_{k}(u)^{2}}{\varepsilon+\left|G_{k}(u)\right|} d x \\
& +C\left\|f \chi_{\left\{f>\frac{\alpha_{0}}{2} h(k)\right\}}\right\|_{L^{m}(\Omega)}\left(\int_{\Omega}\left(\frac{G_{k}(u)^{2}}{\varepsilon+\left|G_{k}(u)\right|}\right)^{p^{*}} d x\right)^{\frac{1}{p^{*}}} .
\end{aligned}
$$

Now observe that

$$
\frac{t^{2}}{1+t} \leq c \int_{0}^{t}\left(\frac{\xi}{1+\xi}\right)^{\frac{1}{p}} d \xi \Longleftrightarrow \frac{s^{2}}{\varepsilon+s} \leq c \varphi_{\varepsilon}(s),
$$


so that we conclude

$$
\begin{aligned}
\int_{\Omega} f \frac{G_{k}(u)^{2}}{\varepsilon+\left|G_{k}(u)\right|} d x \leq & \frac{\alpha_{0}}{2} \int_{\Omega} h(|u|) \frac{G_{k}(u)^{2}}{\varepsilon+\left|G_{k}(u)\right|} d x \\
& +C\left\|f \chi_{\left\{f>\frac{\alpha_{0}}{2} h(k)\right\}}\right\|_{L^{m}(\Omega)}\left\|\varphi_{\varepsilon}\left(G_{k}(u)\right)\right\|_{L^{p^{*}}(\Omega)} .
\end{aligned}
$$

The term containing $g$ is also dealt with as we did in (3.14)-(3.15); we obtain

$$
\begin{aligned}
2 \int_{\Omega}|g| \frac{G_{k}(u)}{\varepsilon+\left|G_{k}(u)\right|} d x \leq & \frac{\alpha_{0}}{2} \int_{\Omega} h(|u|) \frac{G_{k}(u)^{2}}{\varepsilon+\left|G_{k}(u)\right|} d x \\
& +2 \frac{\delta_{k}}{\varepsilon+\delta_{k}}\|g\|_{L^{1}(\Omega)}+2 \int_{\left\{|g|>\frac{\alpha_{0}}{4} h(k) \delta_{k}\right\}}|g| d x,
\end{aligned}
$$

and choosing $\delta_{k}=\frac{\alpha_{0}}{\sqrt{h(k)}}$, we get

$$
\begin{aligned}
2 \int_{\Omega}|g| \frac{G_{k}(u)}{\varepsilon+\left|G_{k}(u)\right|} d x \leq & \frac{\alpha_{0}}{2} \int_{\Omega} h(|u|) \frac{G_{k}(u)^{2}}{\varepsilon+\left|G_{k}(u)\right|} d x \\
& +\frac{2}{\varepsilon} \frac{\alpha_{0}}{\sqrt{h(k)}}\|g\|_{L^{1}(\Omega)}+2\left\||g| \chi_{\left\{|g|>\frac{\alpha_{0}^{2}}{4} \sqrt{h(k)}\right\}}\right\| L_{L^{1}(\Omega)} .
\end{aligned}
$$

We have obtained, from (3.28)-(3.32),

$$
\begin{aligned}
& \alpha\left\|\varphi_{\varepsilon}\left(G_{k}(u)\right)\right\|_{W_{0}^{1, p}(\Omega)}^{p} \\
& \leq \gamma C\left\|\varphi_{\varepsilon}\left(G_{k}(u)\right)\right\|_{W_{0}^{1, p}(\Omega)}^{q}\left(\left\|\varphi_{\varepsilon}\left(G_{k}(u)\right)\right\|_{W_{0}^{1, p}(\Omega)}^{p^{*}\left(1-\frac{q}{p}\right)}+\varepsilon^{p^{*}\left(1-\frac{q}{p}\right)}\right) \\
& \quad+C\left\|f \chi_{\left\{f>\frac{\alpha_{0}}{2} h(k)\right\}}\right\|_{L^{m}(\Omega)}\left\|\varphi_{\varepsilon}\left(G_{k}(u)\right)\right\|_{W_{0}^{1, p}(\Omega)} \\
& \quad+\frac{2}{\varepsilon} \frac{\alpha_{0}}{\sqrt{h(k)}}\|g\|_{L^{1}(\Omega)}+2\left\||g| \chi_{\left\{|g|>\frac{\alpha_{0}^{2}}{2} \sqrt{h(k)}\right\}}\right\|_{L^{1}(\Omega)},
\end{aligned}
$$

which yields, using Young's inequality,

$$
\begin{aligned}
& \frac{\alpha}{2}\left\|\varphi_{\varepsilon}\left(G_{k}(u)\right)\right\|_{W_{0}^{1, p}(\Omega)}^{p} \\
& \leq \gamma C\left\|\varphi_{\varepsilon}\left(G_{k}(u)\right)\right\|_{W_{0}^{1, p}(\Omega)}^{q}\left(\left\|\varphi_{\varepsilon}\left(G_{k}(u)\right)\right\|_{W_{0}^{1, p}(\Omega)}^{p^{*}\left(1-\frac{q}{p}\right)}+\varepsilon^{p^{*}\left(1-\frac{q}{p}\right)}\right) \\
& \quad+C \alpha^{-\frac{1}{p-1}\left\|f \chi_{\left\{f>\frac{\alpha_{0}}{2} h(k)\right\}}\right\|_{L^{m}(\Omega)}^{p^{\prime}}} \\
& \quad+\frac{2}{\varepsilon} \frac{\alpha_{0}}{\sqrt{h(k)}}\|g\|_{L^{1}(\Omega)}+2\left\||g| \chi_{\left\{|g|>\frac{\alpha_{0}^{2}}{2} \cdot \sqrt{h(k)\}}\right.}\right\|_{L^{1}(\Omega)}
\end{aligned}
$$


Set now

$$
Y_{k}=\left\|\varphi_{\varepsilon}\left(G_{k}(u)\right)\right\|_{W_{0}^{1, p}(\Omega)} .
$$

Then, the previous inequality implies, for some constants $C_{1}, C_{2}>0$,

$$
\begin{aligned}
C_{1} \alpha Y_{k}^{p}-\gamma C_{2} Y_{k}^{q}\left(Y_{k}^{p^{*}\left(1-\frac{q}{p}\right)}+\varepsilon^{p^{*}\left(1-\frac{q}{p}\right)}\right) \leq & \alpha^{-\frac{1}{p-1}}\left\|f \chi_{\left\{f>\frac{\alpha_{0}}{2} h(k)\right\}}\right\|_{L^{m}(\Omega)}^{p^{\prime}} \\
& +\frac{2}{\varepsilon} \frac{\alpha_{0}}{\sqrt{h(k)}}\|g\|_{L^{1}(\Omega)} \\
& +2\left\||g| \chi_{\left\{|g|>\frac{\alpha_{0}^{2}}{2} \sqrt{h(k)}\right\}}\right\|_{L^{1}(\Omega)} .
\end{aligned}
$$

Note that if either $g=0$ or $\alpha_{0}=0$ we could have taken $\varepsilon=0$ and in that case (3.33) is nothing but the inequality (3.16) with $m=\left(p^{*}\right)^{\prime}$ and $r=1$, namely this is the proof given in the previous case. Otherwise, (3.33) should be seen as an $\varepsilon$-perturbation of (3.16). In particular, using Young's inequality we get

$$
\begin{aligned}
C_{1} \alpha Y_{k}^{p}-\gamma C_{3} Y_{k}^{q+p^{*}\left(1-\frac{q}{p}\right)} \leq & \gamma C_{4} \varepsilon^{q+p^{*}\left(1-\frac{q}{p}\right)}+\alpha^{-\frac{1}{p-1}}\left\|f \chi_{\left\{f>\frac{\alpha_{0}}{2} h(k)\right\}}\right\|_{L^{m}(\Omega)}^{p^{\prime}} \\
& +\frac{1}{\varepsilon} \frac{\alpha_{0}}{\sqrt{h(k)}}\|g\|_{L^{1}(\Omega)}+\left\||g| \chi_{\left\{|g|>\frac{\alpha_{0}^{2}}{2} \sqrt{h(k)}\right\}}\right\|_{L^{1}(\Omega)} .
\end{aligned}
$$

Here we take $\varepsilon$ small, and then choose $k$ large enough, so that the right-hand side is smaller than $F^{*}=\max _{Y \in(0, \infty)} C_{1} \alpha Y^{p}-\gamma C_{3} Y^{q+p^{*}\left(1-\frac{q}{p}\right)}$. Then, we conclude following the previous proof.

Note that both Remarks 3.5 and 3.6 remain true as well.

As a consequence of the previous estimates, we can obtain an existence result for problem (2.1). However, to this purpose we need to make some extra assumptions to control the growth of the lower order terms. We will assume that

$$
\forall L>0 \quad \exists \gamma_{L} \in L^{1}(\Omega): \quad\left|a_{0}(x, s)\right| \leq \gamma_{L}(x) \quad \forall s:|s| \leq L,
$$

and we strengthen hypothesis (2.6) assuming that

$$
\left\{\begin{array}{l}
|H(x, s, \xi)| \leq \gamma|\xi|^{q}+f(x) \\
\gamma>0, \quad p-1<q<p, \quad f \in L^{m}(\Omega), \quad m \geq 1
\end{array}\right.
$$

where the exponents $q$ and $m$ still satisfy (2.7).

Let us point out that (3.35) is not the most general assumption we could choose. Indeed, the previous a priori estimate only requires (2.6), which can be complemented by assuming that $H(x, u, D u) \operatorname{sign}(u)$ has natural growth from below $(e . g$. $H(x, u, D u) \operatorname{sign}(u) \geq-\beta(u)|D u|^{p}-f(x)$ for some continuous function $\left.\beta(s)\right)$. 
However, such generality would require some more (technical though nowadays well known) compactness arguments in the proof below, which is beyond the goals of this paper. The interested reader is referred to [7] and to its subsequent extensions for what concerns lower order terms with natural growth and "good sign".

Theorem 3.7. Assume (2.2)-(2.7) and (3.34), (3.35), with $p-1+\frac{p}{N} \leq q<p$, and that either

$$
\text { (i) } \alpha_{0}>0
$$

or

(ii) $\alpha_{0}=0$ and (3.2) holds true.

Then there exists $u \in W_{0}^{1, p}(\Omega)$, satisfying (3.3), which is a weak solution of (2.1) in the sense of (3.1).

Proof. We consider a suitable approximate problem, replacing $H(x, s, \xi)$ with its truncation $T_{n}(H(x, s, \xi))$. By classical results $(e . g$. [33]), there exists a solution $u_{n} \in W_{0}^{1, p}(\Omega)$ of the problem

$$
\left\{\begin{array}{l}
-\operatorname{div}\left(a\left(x, u_{n}, D u_{n}\right)\right)+a_{0}\left(x, u_{n}\right)=T_{n}\left(H\left(x, u_{n}, D u_{n}\right)\right) \text { in } \Omega \\
u_{n}=0 \text { on } \partial \Omega
\end{array}\right.
$$

First of all, at $n$ fixed we show that $u_{n}$ satisfies (3.3). Note that the only case to be considered is that $q>p-1+\frac{p}{N}$, where $\sigma>1$ and (3.3) is a stronger condition than $u_{n} \in W_{0}^{1, p}(\Omega)$. The proof that (3.3) is satisfied by any solution $u_{n} \in W_{0}^{1, p}(\Omega)$ follows the lines of the classical regularity results by Stampacchia ( [41]); for the sake of clarity, we prove this claim here. Let us take $\left|T_{k}\left(u_{n}\right)\right|^{\lambda-1} T_{k}\left(u_{n}\right)$ as test function in (3.36), where $\lambda$ is the same exponent given by (3.6). Using assumptions (2.2) and (2.5) we get

$$
\begin{aligned}
& \alpha \lambda \int_{\Omega}\left|D T_{k}\left(u_{n}\right)\right|^{p}\left|T_{k}\left(u_{n}\right)\right|^{\lambda-1} d x \\
& \leq \int_{\Omega} T_{n}\left(H\left(x, u_{n}, D u_{n}\right)\right)\left|T_{k}\left(u_{n}\right)\right|^{\lambda-1} T_{k}\left(u_{n}\right) d x+\lambda \int_{\Omega}|g|\left|T_{k}\left(u_{n}\right)\right|^{\lambda-1} d x,
\end{aligned}
$$

which yields

$$
\begin{aligned}
& \int_{\Omega}\left|D T_{k}\left(u_{n}\right)\right|^{p}\left|T_{k}\left(u_{n}\right)\right|^{\lambda-1} d x \\
& \leq C n \int_{\Omega}\left|T_{k}\left(u_{n}\right)\right|^{\lambda} d x+C\|g\|_{L^{r}(\Omega)}\left(\int_{\Omega}\left|T_{k}\left(u_{n}\right)\right|^{(\lambda-1) r^{\prime}} d x\right)^{\frac{1}{r^{\prime}}} .
\end{aligned}
$$

Using that $\frac{(\lambda-1)}{p}+1=\sigma($ see $(3.10))$, we have

$$
\int_{\Omega}\left|D T_{k}\left(u_{n}\right)\right|^{p}\left|T_{k}\left(u_{n}\right)\right|^{\lambda-1} d x=\left.\left.C \int_{\Omega}|D| T_{k}\left(u_{n}\right)\right|^{\sigma}\right|^{p} d x
$$


and due to Sobolev embedding we obtain

$$
\begin{aligned}
\left(\int_{\Omega}\left|T_{k}\left(u_{n}\right)\right|^{\sigma p^{*}} d x\right)^{\frac{p}{p^{*}}} \leq & \left.\left.C \int_{\Omega}|D| T_{k}\left(u_{n}\right)\right|^{\sigma}\right|^{p} d x \\
\leq & C n \int_{\Omega}\left|T_{k}\left(u_{n}\right)\right|^{\lambda} d x \\
& +C\|g\|_{L^{r}(\Omega)}\left(\int_{\Omega}\left|T_{k}\left(u_{n}\right)\right|^{(\lambda-1) r^{\prime}} d x\right)^{\frac{1}{r^{\prime}}},
\end{aligned}
$$

hence

$$
\begin{aligned}
\left(\int_{\Omega}\left|T_{k}\left(u_{n}\right)\right|^{\sigma p^{*}} d x\right)^{\frac{p}{p^{*}}} \leq & \left.\left.C \int_{\Omega}|D| T_{k}\left(u_{n}\right)\right|^{\sigma}\right|^{p} d x \\
\leq & C n\left(\int_{\Omega}\left|T_{k}\left(u_{n}\right)\right|^{\sigma p^{*}} d x\right)^{\frac{\lambda}{\sigma p^{*}}} \\
& +C\|g\|_{L^{r}(\Omega)}\left(\int_{\Omega}\left|T_{k}\left(u_{n}\right)\right|^{(\lambda-1) r^{\prime}} d x\right)^{\frac{1}{r^{\prime}}} .
\end{aligned}
$$

One can check that the values of $r$ and $\lambda$ imply (as already used in Theorem 3.1) that $(\lambda-1) r^{\prime}=\sigma p^{*}$ and, moreover, we have $\frac{\lambda}{\sigma p^{*}}<\frac{p}{p^{*}}$ (because $\sigma=\frac{(\lambda-1)}{p}+1$ ) and $\frac{1}{r^{\prime}}<\frac{p}{p^{*}}$ (because $r<\frac{N}{p}$ ). Therefore, (3.37) implies an estimate on $\left|T_{k}\left(u_{n}\right)\right|^{\sigma}$ in $W_{0}^{1, p}(\Omega)$ which is uniform in $k$; letting $k$ tend to infinity we deduce that $\left|u_{n}\right|^{\sigma} \in$ $W_{0}^{1, p}(\Omega)$. Thus, we have proved that $u_{n}$ satisfies (3.3) and now Theorem 3.1 applies, giving an estimate, which is uniform in $n$, both for $u_{n}$ and for $\left|u_{n}\right|^{\sigma}$ in $W_{0}^{1, p}(\Omega)$.

In particular, $\left|D u_{n}\right|^{q}$ is bounded in $L^{\frac{p}{q}}(\Omega)$, hence, thanks to the growth condition (3.35), we deduce that $T_{n}\left(H\left(x, u_{n}, D u_{n}\right)\right)$ is bounded in $L^{\tau}(\Omega)$ with $\tau=$ $\min \left(\frac{p}{q}, m\right)$. Since $u_{n}$ is bounded in $W_{0}^{1, p}(\Omega)$ and the right-hand side is bounded in $L^{1}(\Omega)$, well-known compactness results (see [14]) imply that, up to extracting a subsequence, $u_{n}$ converges weakly to some $u \in W_{0}^{1, p}(\Omega)$ with $D u_{n}$ which converges to $D u$ almost everywhere in $\Omega$. In turn this implies that $a\left(x, u_{n}, D u_{n}\right)$ converges weakly to $a(x, u, D u)$ in $L^{p^{\prime}}(\Omega)^{N}$, and that $T_{n}\left(H\left(x, u_{n}, D u_{n}\right)\right)$ converges to $H(x, u, D u)$ strongly in $L^{\tau^{\prime}}(\Omega)$ for every $\tau^{\prime}<\min \left(\frac{p}{q}, m\right)$.

In particular, the term $T_{n}\left(H\left(x, u_{n}, D u_{n}\right)\right)$ strongly converges in $L^{1}(\Omega)$, which together with assumption (3.34) yields the compactness in $L^{1}(\Omega)$ of $a_{0}\left(x, u_{n}\right)$. We can therefore pass to the limit in the approximate equation. This proves the existence of a solution of (3.1) which also lies in the class (3.3).

In the Introduction we already pointed out, on account of Example 1.3, that condition (3.3) is a threshold for a bootstrap argument to work. Indeed, we prove 
now that, when the solutions satisfy (3.3), then the classical regularity results known in the "sublinear" case $q \leq p-1$ can be recovered. Namely, if the data $f$ in (2.6) have more summability, then the solutions of (3.1) satisfying (3.3) have also better summability.

Theorem 3.8. Assume (2.2), (2.3), (2.5), (2.6), (2.7) with $p-1+\frac{p}{N} \leq q<p$. Assume in addition that $f$ belongs to $L^{s}(\Omega)$, with $s>\frac{N(q-(p-1))}{q}$ and $g$ belongs to $L^{t}(\Omega)$, with $t>\frac{N(q-(p-1))}{p}$.

Let $u \in W_{0}^{1, p}(\Omega)$ be a solution of (3.1) which satisfies (3.3). Then we have

(i) If $s>\frac{N}{p}$ and $t>\frac{N}{p}$, then $u \in L^{\infty}(\Omega)$ and

$$
\|u\|_{L^{\infty}(\Omega)} \leq M .
$$

(ii) If $s=t=\frac{N}{p}$, then $u \in L^{d}(\Omega)$ for any $d<\infty$ and

$$
\|u\|_{L^{d}(\Omega)} \leq M
$$

(iii) If $s<\frac{N}{p}$ and $t \geq \frac{N s(p-1)}{p(N-s)}$, then $|u|^{\tau} \in W_{0}^{1, p}(\Omega), u \in L^{\left(s^{*}(p-1)\right)^{*}}(\Omega)$ and

$$
\left\||u|^{\tau}\right\|_{W_{0}^{1, p}(\Omega)}+\|u\|_{L^{\left(s^{*}(p-1)\right)^{*}(\Omega)}} \leq M, \quad \tau=\frac{s(N-p)(p-1)}{p(N-s p)} .
$$

In (3.38)-(3.40), the constant $M$ depends on $p, q, s, t, N, \alpha_{0}, \alpha, \gamma,|\Omega|, d$ (in case (3.39)) and on $\|f\|_{L^{s}(\Omega)}$ and $\|g\|_{L^{t}(\Omega)}$ and, in the case $\alpha_{0}=0$, on $\|u\|_{L^{1}(\Omega)}$ as well.

Remark 3.9. The statement of the a priori estimates (3.38)-(3.40) differs from the estimate in Theorem 3.1 in two points.

Firstly, here the constant $M$ depends on $f, g$ only through $\|f\|_{L^{s}(\Omega)}$ and $\|g\|_{L^{t}(\Omega)}$. This is due to the fact that the exponents $s$ and $t$ are strictly bigger than the limiting values ( $m=\frac{N(q-(p-1))}{q}$ and $r=\frac{N(q-(p-1))}{p}$ respectively) considered in Theorem 3.1, hence a bounded set in $L^{s}(\Omega)$ is also equi-integrable in $L^{m}(\Omega)$, and the same for $L^{t}(\Omega)$ with respect to $L^{r}(\Omega)$.

Secondly, when $\alpha_{0}=0$, the constant $M$ depends also on $\|u\|_{L^{1}(\Omega)}$. This is due to the fact that we did not require condition (3.2), and we stated the result in the viewpoint of a bootstrap regularity. Of course, if we require in addition the size condition (3.2) (concerning the norm of $f$ in $L^{m}(\Omega)$ and of $g$ in $L^{r}(\Omega)$ respectively) then $\|u\|_{L^{1}(\Omega)}$ can be estimated from Theorem 3.1 and the constant $M$ will be estimated in terms of $f$ and $g$ only.

Proof. If we choose $\varphi=G_{k}(u)$ as test function in (3.1), and using (2.2), (2.5), (2.6) we obtain

$\alpha \int_{\Omega}\left|D G_{k}(u)\right|^{p} d x \leq \gamma \int_{\Omega}|D u|^{q} G_{k}(u) d x+\int_{\Omega} f G_{k}(u) d x+\int_{\{|u|>k\}} g d x$. 
Let us remark again that the choice $\varphi=G_{k}(u)$ is not admissible a priori in (3.1) and that the previous inequality is justified using similar arguments as in the beginning of the proof of Theorem 3.1. Using Hölder's inequality we have

$$
\int_{\Omega}|D u|^{q} G_{k}(u) d x \leq\left(\int_{\Omega}\left|D G_{k}(u)\right|^{p} d x\right)^{\frac{q}{p}}\left(\int_{\Omega}\left|G_{k}(u)\right|^{\frac{p}{p-q}} d x\right)^{1-\frac{q}{p}} .
$$

Now observe that since $p-1+\frac{p}{N} \leq q<p$ we have $p^{*} \leq \frac{p}{p-q}<\sigma p^{*}$, where $\sigma$ is the exponent in (3.3). More precisely, one can check that $\frac{p}{p-q}=\left(1-\frac{p}{N}\right) p^{*}+$ $\frac{p}{N}\left(\sigma p^{*}\right)$. By the interpolation inequality, and then by Sobolev embedding, we get

$$
\begin{aligned}
\left(\int_{\Omega}\left|G_{k}(u)\right|^{\frac{p}{p-q}} d x\right)^{1-\frac{q}{p}} & \leq\left(\int_{\Omega}\left|G_{k}(u)\right|^{p^{*}} d x\right)^{\frac{p-q}{p^{*}}}\left(\int_{\Omega}\left|G_{k}(u)\right|^{\sigma p^{*}} d x\right)^{\frac{p-q}{N}} \\
& \leq C\left(\int_{\Omega}\left|D G_{k}(u)\right|^{p} d x\right)^{\frac{p-q}{p}}\left(\int_{\Omega}\left|G_{k}(u)\right|^{\sigma p^{*}} d x\right)^{\frac{p-q}{N}} .
\end{aligned}
$$

Together with (3.42) we obtain then from (3.41):

$$
\begin{aligned}
\alpha \int_{\Omega}\left|D G_{k}(u)\right|^{p} d x \leq & \gamma C\left(\int_{\Omega}\left|D G_{k}(u)\right|^{p} d x\right)\left(\int_{\Omega}\left|G_{k}(u)\right|^{\sigma p^{*}} d x\right)^{\frac{p-q}{N}} \\
& +\int_{\Omega} f G_{k}(u) d x+\int_{\{|u|>k\}} g d x
\end{aligned}
$$

which yields, since $\frac{p-q}{N}=\frac{q-(p-1)}{\sigma p^{*}}$,

$$
\left(\alpha-\gamma C\left\|G_{k}(u)\right\|_{L^{\sigma p^{*}}(\Omega)}^{q-(p-1)}\right) \int_{\Omega}\left|D G_{k}(u)\right|^{p} d x \leq \int_{\Omega} f G_{k}(u) d x+\int_{\{|u|>k\}} g d x .
$$

Let $k_{0}$ be such that

$$
\gamma C\left\|G_{k}(u)\right\|_{L^{\sigma p^{*}(\Omega)}}^{q-(p-1)} \leq \frac{\alpha}{2} \quad \forall k \geq k_{0}
$$

Then we get

$$
\frac{\alpha}{2} \int_{\Omega}\left|D G_{k}(u)\right|^{p} d x \leq \int_{\Omega} f G_{k}(u) d x+\int_{\{|u|>k\}} g d x \quad \forall k>k_{0} .
$$

Now, if $f, g \in L^{\frac{N}{p}+\delta}(\Omega)$ for some $\delta>0$, we obtain from (3.44) that $u \in L^{\infty}(\Omega)$ by applying the method of G. Stampacchia (see [41]). 
If $f \in L^{s}(\Omega)$ with $\left(p^{*}\right)^{\prime}<s<\frac{N}{p}, g \in L^{t}(\Omega)$ with $t=\frac{N s(p-1)}{(N-s) p}$, we take as test function (after the usual approximation) $\psi\left(G_{k}(u)\right)$, where

$$
\psi(s)=\int_{0}^{s} T_{n}(\xi)^{(\tau-1) p} d \xi
$$

where $\tau>1$ will be chosen later. Let us stress that the truncation $T_{n}$ is used here because we give a regularity result and not only a priori estimates.

Using (2.2), (2.5), (2.6), we obtain:

$$
\begin{aligned}
\alpha \int_{\Omega}\left|D G_{k}(u)\right|^{p} T_{n}\left(G_{k}(u)\right)^{(\tau-1) p} d x \leq & \gamma \int_{\Omega}|D u|^{q} \psi\left(G_{k}(u)\right) d x \\
& +\int_{\Omega} f \psi\left(G_{k}(u)\right) d x \\
& +\int_{\{|u|>k\}} g T_{n}\left(G_{k}(u)\right)^{(\tau-1) p} d x .
\end{aligned}
$$

Since we have

$$
\begin{aligned}
\psi(s) & =\int_{0}^{s} T_{n}(\xi)^{(\tau-1) p} d \xi \leq T_{n}(s)^{(\tau-1) q} \int_{0}^{s} T_{n}(\xi)^{(\tau-1)(p-q)} d \xi \\
& \leq T_{n}(s)^{(\tau-1) q}\left(\int_{0}^{s} T_{n}(\xi)^{\tau-1} d \xi\right)^{p-q} s^{q-(p-1)},
\end{aligned}
$$

we get

$$
\begin{aligned}
& \int_{\Omega}|D u|^{q} \psi\left(G_{k}(u)\right) d x \\
& \leq \int_{\Omega}|D u|^{q} T_{n}\left(G_{k}(u)\right)^{(\tau-1) q}\left(\int_{0}^{G_{k}(u)} T_{n}(\xi)^{\tau-1} d \xi\right)^{p-q} G_{k}(u)^{q-(p-1)} d x .
\end{aligned}
$$

Using Hölder inequality with the three exponents $\frac{p}{q}, \frac{p^{*}}{p-q}, \frac{N}{p-q}$ we obtain

$$
\begin{aligned}
& \int_{\Omega}|D u|^{q} \psi\left(G_{k}(u)\right) d x \leq\left(\int_{\Omega}|D u|^{p} T_{n}\left(G_{k}(u)\right)^{(\tau-1) p} d x\right)^{\frac{q}{p}} \\
& \times\left(\int_{\Omega}\left(\int_{0}^{G_{k}(u)} T_{n}(\xi)^{\tau-1} d \xi\right)^{p^{*}} d x\right)^{\frac{1}{p^{*}(p-q)}}\left(\int_{\Omega} G_{k}(u)^{\frac{N(q-(p-1))}{p-q}} d x\right)^{\frac{p-q}{N}},
\end{aligned}
$$


and we conclude, using Sobolev embedding and recalling (see (3.3)) the value of $\sigma=\frac{(N-p)(q-(p-1))}{p(p-q)}$ :

$$
\int_{\Omega}|D u|^{q} \psi\left(G_{k}(u)\right) d x \leq C\left(\int_{\Omega}|D u|^{p} T_{n}\left(G_{k}(u)\right)^{(\tau-1) p} d x\right)\left\|G_{k}(u)\right\|_{L^{\sigma p^{*}}(\Omega)}^{q-(p-1)} .
$$

We obtain then from $(3.45)$

$$
\begin{aligned}
& \left(\alpha-\gamma C\left\|G_{k}(u)\right\|_{L^{\sigma} p^{*}(\Omega)}^{q-(p-1)}\right) \\
& \int_{\Omega}\left|D G_{k}(u)\right|^{p} T_{n}\left(G_{k}(u)\right)^{(\tau-1) p} d x \\
& \leq \int_{\Omega} f \psi\left(G_{k}(u)\right) d x+\int_{\{|u|>k\}} g T_{n}\left(G_{k}(u)\right)^{(\tau-1) p} d x .
\end{aligned}
$$

Choosing $k_{0}$ as in (3.43) yields

$$
\frac{\alpha}{2} \int_{\Omega}\left|D \Phi\left(G_{k}(u)\right)\right|^{p} d x \leq \int_{\Omega} f \psi\left(G_{k}(u)\right) d x+\int_{\{|u|>k\}} g T_{n}\left(G_{k}(u)\right)^{(\tau-1) p} d x,
$$

where $\Phi(s)=\int_{0}^{s} T_{n}(\xi)^{\tau-1} d \xi$. Now, since $\Phi(s) \geq \frac{1}{\tau} T_{n}(u)^{\tau-1} u$, we have

$$
T_{n}(s)^{(\tau-1) p} \leq T_{n}(s)^{(\tau-1) p}\left(\frac{s}{T_{n}(s)}\right)^{\frac{(\tau-1) p}{\tau}} \leq C \Phi(s)^{\frac{(\tau-1) p}{\tau}},
$$

and in a similar way we get

$$
\psi(s)=\int_{0}^{s} T_{n}(\xi)^{(\tau-1) p} d \xi \leq C \Phi(s)^{\frac{(\tau-1) p+1}{\tau}} .
$$

Therefore we have

$$
\begin{aligned}
& \frac{\alpha}{2} \int_{\Omega}\left|D \Phi\left(G_{k}(u)\right)\right|^{p} d x \\
& \leq \int_{\Omega}|f|\left|\Phi\left(G_{k}(u)\right)\right|^{\frac{(\tau-1) p+1}{\tau}} d x+\int_{\{|u|>k\}}|g|\left|\Phi\left(G_{k}(u)\right)\right|^{\frac{(\tau-1) p}{\tau}} d x,
\end{aligned}
$$

and using Hölder and Sobolev inequality we obtain

$$
\begin{aligned}
\frac{\alpha}{2} C\left(\int_{\Omega}\left|\Phi\left(G_{k}(u)\right)\right|^{p^{*}} d x\right)^{\frac{p}{p^{*}}} & \leq \frac{\alpha}{2} \int_{\Omega}\left|D \Phi\left(G_{k}(u)\right)\right|^{p} d x \\
\leq & \|f\|_{L^{s}(\Omega)}\left(\int_{\Omega}\left|\Phi\left(G_{k}(u)\right)\right|^{\left[\frac{(\tau-1) p+1}{\tau}\right] s^{\prime}} d x\right)^{\frac{1}{s^{\prime}}} \\
& +\|g\|_{L^{t}(\Omega)}\left(\int_{\Omega}\left|\Phi\left(G_{k}(u)\right)\right|^{\left[\frac{(\tau-1) p}{\tau}\right] t^{\prime}} d x\right)^{\frac{1}{t^{\prime}}},
\end{aligned}
$$


where $t=\frac{N s(p-1)}{(N-s) p}$. We choose now $\tau$ such that $\left[\frac{(\tau-1) p+1}{\tau}\right] s^{\prime}=p^{*}$. The reader can check that the values of $t$ and $\tau$ also imply $\left[\frac{(\tau-1) p}{\tau}\right] t^{\prime}=p^{*}$. Therefore we obtain an estimate on $\Phi\left(G_{k}(u)\right)$ in $L^{p^{*}}(\Omega)$, which means, by definition of $\Phi$, an estimate on $T_{n}(u)$ in $L^{\tau} p^{*}(\Omega)$. Finally letting $n$ tend to infinity one obtains that $u \in L^{\tau} p^{*}(\Omega)$ and actually, from (3.46), that $u^{\tau} \in W_{0}^{1, p}(\Omega)$.

Note that, since $\left(s^{*}(p-1)\right)^{*}=\frac{N s(p-1)}{N-p s}$ tends to infinity as $s$ tends to $\frac{N}{p}$, we can deduce case (ii) from case (iii).

Moreover, in all cases (i)-(iii), we also obtain from the above proof corresponding estimates on $u$ depending on $\|f\|_{L^{s}(\Omega)},\|g\|_{L^{t}(\Omega)}$ and on the value $k_{0}$ given by (3.43).

On the other hand, if $\alpha_{0}>0$, Remark 3.5 implies that $k_{0}$ only depends on the bound and on the equi-integrability of $f$ in $L^{m}(\Omega)$ and of $g$ in $L^{r}(\Omega)$, where $m=\frac{N(q-(p-1))}{q}, r=\frac{N(q-(p-1))}{p}$. Since $s>m$ and $t>r$, we deduce that $k_{0}$ can be estimated in terms of $\|f\|_{L^{s}(\Omega)}$ and $\|g\|_{L^{t}(\Omega)}$ only.

If $\alpha_{0}=0$, using Remark 3.6 we have that $k_{0}$ can be estimated in terms of $\|f\|_{L^{s}(\Omega)},\|g\|_{L^{t}(\Omega)}$ and $\|u\|_{L^{1}(\Omega)}$ as well.

\section{The case $\frac{N(p-1)}{N-1} \leq q<p-1+\frac{p}{N}$}

In this section we consider the range of values $\frac{N(p-1)}{N-1} \leq q<p-1+\frac{p}{N}$. In that case, it follows from (2.6)-(2.8) that it is possible to take data $f \in L^{m}(\Omega)$ with $m<\left(p^{*}\right)^{\prime}$, so that they may not belong to $W^{-1, p^{\prime}}(\Omega)$. Therefore, we expect to find solutions which are not in $W_{0}^{1, p}(\Omega)$. In this case, the usual energy formulation (3.1) can not be used, and, on the other hand, the simple distributional setting is usually not strong enough to obtain estimates (or uniqueness, if ever). We will adopt a socalled renormalized formulation (see $[11,22]$ ), which relies on the requirement that the truncations $T_{k}(u)$ belong to $W_{0}^{1, p}(\Omega)$ for every $k>0$. More precisely, we call $u$ a renormalized solution if it satisfies the following definition.

Definition 4.1. A function $u: \Omega \rightarrow \mathbb{R}$ which is finite almost everywhere is a renormalized solution of $(2.1)$ if it satisfies ${ }^{3}$

$$
\left\{\begin{array}{l}
T_{k}(u) \in W_{0}^{1, p}(\Omega) \quad \forall k>0, \quad a_{0}(x, u) \in L^{1}(\Omega), \quad H(x, u, D u) \in L^{1}(\Omega), \\
\int_{\Omega} a(x, u, D u) D(S(u) \varphi) d x+\int_{\Omega} a_{0}(x, u) S(u) \varphi d x=\int_{\Omega} H(x, u, D u) S(u) \varphi d x \\
\text { for any Lipschitz function } S: \mathbb{R} \rightarrow \mathbb{R} \text { having compact support } \\
\text { and for any } \varphi \in W^{1, p}(\Omega) \cap L^{\infty}(\Omega) \text { such that } S(u) \varphi \in W_{0}^{1, p}(\Omega) .
\end{array}\right.
$$

${ }^{3}$ By $D u$ we denote here the generalized gradient of $u$ (see $[6,22]$ ) which is defined, roughly speaking, as $D u=D T_{k}(u) \chi_{\{|u|<k\}}$, for any $k>0$. 
Remark 4.2. We warn the reader that in the notion of renormalized solution which is currently used in case of $L^{1}$-data (see e.g. [22]) (4.1) is usually complemented with some requirement concerning the behaviour of the energy in the set where $|u|=+\infty$, for example by requiring that

$$
\lim _{n \rightarrow \infty} \frac{1}{n} \int_{\{n<|u|<2 n\}} a(x, u, D u) D u d x=0 .
$$

However, in the range of the present section we deal with data $f$ in $L^{m}(\Omega), m>1$, and with solutions satisfying the regularity condition (4.4) below. Since condition (4.2) is an obvious consequence of (4.4), we do not need here to add such a condition in our definition of renormalized solution. This is the same spirit adopted in [11], where the notion of renormalized solution was introduced for elliptic problems requiring only (4.1) (and not also (4.2)) to hold.

Up to this change of setting due to the fact that solutions can possibly have infinite energy, our result concerning a priori estimates sounds like Theorem 3.1 in the previous section.

Theorem 4.3. Assume (2.2), (2.3), (2.5), (2.6), (2.7), with $\frac{N(p-1)}{N-1}<q<p-1+\frac{p}{N}$ (hence in (2.7) we have $\left.m=\frac{N(q-(p-1))}{q}, r=1\right)$. Assume further that either

(i) $\alpha_{0}>0$,

or

(ii) $\alpha_{0}=0$ and

$$
\alpha^{-\frac{p^{*}}{p m^{\prime}-p^{*}}}\|f\|_{L^{m}(\Omega)}^{\frac{p m^{\prime}}{p m^{\prime}-p^{*}}}+\kappa_{0}\|g\|_{L^{1}(\Omega)}^{1-\theta} \gamma^{\theta} \leq C^{*} \alpha^{\frac{N-q}{p-q}} \gamma^{-\frac{N-p}{p-q}}
$$

where $\theta=\frac{p(1-\sigma)}{q+1}>0$, and $\kappa_{0}, C^{*}$ are constants depending only on $p, N, q$ which will be defined in (4.14), (4.13) below.

Let $u$ be a renormalized solution, in the sense of Definition 4.1, which satisfies the regularity condition

$$
(1+|u|)^{\sigma-1} u \in W_{0}^{1, p}(\Omega), \quad \sigma=\frac{(N-p)(q-(p-1))}{p(p-q)} .
$$

Then we have

$$
\left\|(1+|u|)^{\sigma-1} u\right\|_{W_{0}^{1, p}(\Omega)}+\left\||D u|^{p-1}\right\|_{L^{\frac{N(q-(p-1))}{p-1}}(\Omega)} \leq M,
$$

where $M$ depends on $p, q, N, \alpha_{0}, \alpha, \gamma,|\Omega|$ and the data $f$ and $g$. When $\alpha_{0}=0$ the constant $M$ does not depend on $|\Omega| ;$ it depends on $f$ and $g$ only through $\|f\|_{L^{m}(\Omega)}$ and $\|g\|_{L^{1}(\Omega)}$ (in particular through the gap in (4.3)). When $\alpha_{0}>0$, the constant $M$ does not depend only on $\|f\|_{L^{m}(\Omega)}$ and $\|g\|_{L^{1}(\Omega)}$ but remains bounded when $f$ and $g$ vary in sets which are bounded and equi-integrable, respectively, in $L^{m}(\Omega)$ and in $L^{1}(\Omega)$. 
Remark 4.4. Recall that since assumption (2.7) implies that $m=\frac{N(q-(p-1))}{q}$, one has $\frac{N(q-(p-1))}{(p-1)}=m^{*}$. Thus (4.5) gives an estimate for $|D u|^{p-1}$ in $L^{m^{*}}(\Omega)$.

Remark 4.5. Remarks 3.2 and 3.3 remain valid as far as Theorem 4.3 is concerned. The same holds for Remark 3.4 if one considers nonnegative renormalized subsolutions.

Note that the energy requirement (4.4) implies, by itself, that the truncations $T_{k}(u)$ belong to $W_{0}^{1, p}(\Omega)$, which is needed to give sense to the renormalized formulation. We also stress that uniqueness results for solutions in the previous setting, i.e. satisfying (4.1) and (4.4), have been proved in $[5,40]$.

Proof of Theorem 4.3. Using standard arguments in the renormalized setting and the regularity (4.4), one can justify the choice in (4.1) of the test functions $S_{n}, \varphi$ given by

$$
S_{n}(u)=\int_{0}^{T_{n}\left(G_{k}(u)\right)}(\varepsilon+|t|)^{p(\sigma-1)} \frac{|t|}{\alpha_{0} \varepsilon+|t|} d t \quad \text { and } \varphi=1,
$$

even if $S_{n}$ has not compact support. Note that we have stressed the difference between the case $\alpha_{0}>0$ and $\alpha_{0}=0$, since in this latter case $S_{n}$ has a simpler form.

We obtain, using (2.2), (2.5) and (2.6),

$$
\begin{aligned}
& \alpha \int_{\Omega}\left|D T_{n}(u)\right|^{p}\left(\varepsilon+\left|T_{n}\left(G_{k}(u)\right)\right|\right)^{p(\sigma-1)} \frac{\left|T_{n}\left(G_{k}(u)\right)\right|}{\alpha_{0} \varepsilon+\left|T_{n}\left(G_{k}(u)\right)\right|} d x \\
& +\alpha_{0} \int_{\Omega} h(|u|)\left|S_{n}(u)\right| d x \leq \gamma \int_{\Omega}|D u|^{q}\left|S_{n}(u)\right| d x \\
& +\int_{\Omega}|f|\left|S_{n}(u)\right| d x+\int_{\Omega} g\left(\varepsilon+\left|T_{n}\left(G_{k}(u)\right)\right|\right)^{p(\sigma-1)} \frac{\left|T_{n}\left(G_{k}(u)\right)\right|}{\alpha_{0} \varepsilon+\left|T_{n}\left(G_{k}(u)\right)\right|} d x .
\end{aligned}
$$

Then we let $n$ tend to infinity, which is allowed thanks to (4.4) and since $f \in$ $L^{m}(\Omega)$, with $m$ given by (2.7). We end up with

$$
\begin{aligned}
& \alpha \int_{\Omega}|D u|^{p}\left(\varepsilon+\left|G_{k}(u)\right|\right)^{p(\sigma-1)} \frac{\left|G_{k}(u)\right|}{\alpha_{0} \varepsilon+\left|G_{k}(u)\right|} d x+\alpha_{0} \int_{\Omega} h(|u|)|S(u)| d x \\
& \leq \gamma \int_{\Omega}|D u|^{q}|S(u)| d x+\int_{\Omega}|f||S(u)| d x \\
& \quad+\int_{\Omega} g\left(\varepsilon+\left|G_{k}(u)\right|\right)^{p(\sigma-1)} \frac{\left|G_{k}(u)\right|}{\alpha_{0} \varepsilon+\left|G_{k}(u)\right|} d x,
\end{aligned}
$$

where $S(u)=\int_{0}^{G_{k}(u)}(\varepsilon+|t|)^{p(\sigma-1)} \frac{|t|}{\alpha_{0} \varepsilon+|t|} d t$. Now we have

$$
\alpha \int_{\Omega}|D u|^{p}\left(\varepsilon+\left|G_{k}(u)\right|\right)^{p(\sigma-1)} \frac{\left|G_{k}(u)\right|}{\alpha_{0} \varepsilon+\left|G_{k}(u)\right|} d x=\alpha \int_{\Omega} \mid D\left(\left.\varphi_{\varepsilon}\left(G_{k}(u)\right)\right|^{p} d x,\right.
$$


where

$$
\varphi_{\varepsilon}(s)=\int_{0}^{s}(\varepsilon+|\xi|)^{\sigma-1}\left(\frac{|\xi|}{\alpha_{0} \varepsilon+|\xi|}\right)^{\frac{1}{p}} d \xi .
$$

Using that $q<p$, we have

$$
\begin{aligned}
& \int_{\Omega}|D u|^{q}|S(u)| d x=\int_{\Omega} \frac{\left|D \varphi_{\varepsilon}\left(G_{k}(u)\right)\right|^{q}|S(u)|}{\left(\varepsilon+\left|G_{k}(u)\right|\right)^{q(\sigma-1)}}\left(\frac{\left|G_{k}(u)\right|}{\alpha_{0} \varepsilon+\left|G_{k}(u)\right|}\right)^{-\frac{q}{p}} d x \\
& \leq\left(\int_{\Omega}\left|D \varphi_{\varepsilon}\left(G_{k}(u)\right)\right|^{p} d x\right)^{\frac{q}{p}} \\
& \quad \times\left(\int_{\Omega}\left[\frac{|S(u)|}{\left(\varepsilon+\left|G_{k}(u)\right|\right)^{q(\sigma-1)}}\right]^{\frac{p}{p-q}}\left(\frac{\left|G_{k}(u)\right|}{\alpha_{0} \varepsilon+\left|G_{k}(u)\right|}\right)^{-\frac{q}{p-q}} d x\right)^{1-\frac{q}{p}} .
\end{aligned}
$$

Now we observe that we have, for some number $c$,

$$
\left[\frac{|S(u)|}{\left(\varepsilon+\left|G_{k}(u)\right|\right)^{q(\sigma-1)}}\right]^{\frac{p}{p-q}}\left(\frac{\left|G_{k}(u)\right|}{\alpha_{0} \varepsilon+\left|G_{k}(u)\right|}\right)^{-\frac{q}{p-q}} \leq c\left(\varphi_{\varepsilon}\left(G_{k}(u)\right)^{p^{*}}+\varepsilon^{\sigma p^{*}}\right) .
$$

Indeed, scaling $\varepsilon$, and using that $p(\sigma-1)+\frac{p}{p-q}=\sigma p^{*}$, the previous inequality reduces to

$$
\begin{aligned}
& {\left[(1+\xi)^{q(1-\sigma)} \int_{0}^{\xi}(1+t)^{p(\sigma-1)} \frac{t}{\alpha_{0}+t} d t\right]^{\frac{p}{p-q}}\left(\frac{\xi}{\alpha_{0}+\xi}\right)^{-\frac{q}{p-q}}} \\
& \quad \leq c\left\{\left[\int_{0}^{\xi}(1+t)^{\sigma-1}\left(\frac{t}{\alpha_{0}+t}\right)^{\frac{1}{p}} d t\right]^{p^{*}}+1\right\} \quad \forall \xi \geq 0,
\end{aligned}
$$

which is clearly true (again since $p(\sigma-1)+\frac{p}{p-q}=\sigma p^{*}$ ) for some $c>0$. Therefore we obtain

$$
\begin{aligned}
\int_{\Omega}|D u|^{q}|S(u)| d x & \leq C\left(\int_{\Omega}\left|D \varphi_{\varepsilon}\left(G_{k}(u)\right)\right|^{p} d x\right)^{\frac{q}{p}}\left(\int_{\Omega} \varphi_{\varepsilon}\left(G_{k}(u)\right)^{p^{*}}+\varepsilon^{\sigma p^{*}}\right)^{1-\frac{q}{p}} \\
& \leq C\left\|\varphi_{\varepsilon}\left(G_{k}(u)\right)\right\|_{W_{0}^{1, p}(\Omega)}^{q}\left(\left\|\varphi_{\varepsilon}\left(G_{k}(u)\right)\right\|_{W_{0}^{1, p}(\Omega)}^{p^{*}\left(1-\frac{q}{p}\right)}+\varepsilon^{\sigma p^{*}\left(1-\frac{q}{p}\right)}\right) .
\end{aligned}
$$

We split the term containing $f$ as in (3.13), obtaining

$$
\int_{\Omega}|f||S(u)| d x \leq \frac{\alpha_{0}}{2} \int_{\Omega} h(|u|)|S(u)| d x+\int_{\left\{|f|>\frac{\alpha_{0}}{2} h(k)\right\}}|f||S(u)| d x .
$$


Here we use the inequality

$$
S(s)=\int_{0}^{s}(\varepsilon+|t|)^{p(\sigma-1)} \frac{|t|}{\alpha_{0} \varepsilon+|t|} d t \leq c \varphi_{\varepsilon}(s)^{\frac{p^{*}}{m^{\prime}}},
$$

which, rescaling $\varepsilon$ and using that $p(\sigma-1)+1=\frac{\sigma p^{*}}{m^{\prime}}$, is equivalent to

$$
\int_{0}^{\xi}(1+|t|)^{p(\sigma-1)} \frac{|t|}{\alpha_{0}+|t|} d t \leq c\left[\int_{0}^{\xi}(1+|t|)^{\sigma-1}\left(\frac{|t|}{\alpha_{0}+|t|}\right)^{\frac{1}{p}} d \xi\right]^{\frac{p^{*}}{m^{\prime}}}
$$

which holds because $\left(1+\frac{1}{p}\right) \frac{p^{*}}{m^{\prime}} \leq 1+\frac{1}{p}<2$ and $p(\sigma-1)+1=\frac{\sigma p^{*}}{m^{\prime}}$.

Therefore, using the Hölder and Sobolev inequalities, we have

$$
\begin{aligned}
\int_{\Omega}|f||S(u)| d x \leq & \frac{\alpha_{0}}{2} \int_{\Omega} h(|u|)|S(u)| d x \\
& +C \int_{\left\{|f|>\frac{\alpha_{0}}{2} h(k)\right\}}|f| \varphi_{\varepsilon}\left(G_{k}(u)\right)^{\frac{p^{*}}{m^{\prime}}} d x \\
\leq & \frac{\alpha_{0}}{2} \int_{\Omega} h(|u|)|S(u)| d x \\
& +C\left\|f \chi_{\left\{|f|>\frac{\alpha_{0}}{2} h(k)\right\}}\right\|_{L^{m}(\Omega)}\left\|\varphi_{\varepsilon}\left(G_{k}(u)\right)\right\|_{W_{0}^{1, p}(\Omega)}^{\frac{p^{*}}{m^{\prime}}}
\end{aligned}
$$

The term containing $g$ is dealt with similarly as in (3.14)-(3.15); since

$$
\left(\varepsilon+\left|G_{k}(u)\right|\right)^{p(\sigma-1)} \frac{\left|G_{k}(u)\right|}{\alpha_{0} \varepsilon+\left|G_{k}(u)\right|} \leq 2 \frac{|S(u)|}{\left|G_{k}(u)\right|},
$$

we have

$$
\begin{aligned}
& \int_{\Omega}|g|\left(\varepsilon+\left|G_{k}(u)\right|\right)^{p(\sigma-1)} \frac{G_{k}(u)}{\alpha_{0} \varepsilon+G_{k}(u)} d x \leq \frac{\alpha_{0}}{2} \int_{\Omega} h(|u|)|S(u)| d x \\
& +\int_{\left\{|g| \geq \frac{\alpha_{0}}{4} h(|u|)\left|G_{k}(u)\right|\right\}}|g|\left(\varepsilon+\left|G_{k}(u)\right|\right)^{p(\sigma-1)} \frac{G_{k}(u)}{\alpha_{0} \varepsilon+G_{k}(u)} d x \\
& \leq \frac{\alpha_{0}}{2} \int_{\Omega} h(|u|)|S(u)| d x+\frac{\delta_{k}}{\alpha_{0} \varepsilon^{p(1-\sigma)+1}}\|g\|_{L^{1}(\Omega)}+\frac{1}{\varepsilon^{p(1-\sigma)}} \int_{\left\{|g|>\frac{\alpha_{0}}{4} h(k) \delta_{k}\right\}}|g| d x .
\end{aligned}
$$


Choosing, for instance, $\delta_{k}=\frac{\alpha_{0}^{2}}{\sqrt{h(k)}}$, we obtain

$$
\begin{gathered}
\int_{\Omega}|g|\left(\varepsilon+\left|G_{k}(u)\right|\right)^{p(\sigma-1)} \frac{G_{k}(u)}{\alpha_{0} \varepsilon+G_{k}(u)} d x \leq \frac{\alpha_{0}}{2} \int_{\Omega} h(|u|) S(|u|) d x \\
+\frac{1}{\varepsilon^{p(1-\sigma)+1}} \frac{\alpha_{0}}{\sqrt{h(k)}}\|g\|_{L^{1}(\Omega)}+\frac{1}{\varepsilon^{p(1-\sigma)}}\left\|g \chi_{\left\{|g|>\frac{\alpha_{0}^{3}}{4} \sqrt{h(k)}\right\}}\right\|_{L^{1}(\Omega)} .
\end{gathered}
$$

We have obtained, from (4.6)-(4.10),

$$
\begin{aligned}
& \alpha\left\|\varphi_{\varepsilon}\left(G_{k}(u)\right)\right\|_{W_{0}^{1, p}(\Omega)}^{p} \\
& \leq \gamma C\left\|\varphi_{\varepsilon}\left(G_{k}(u)\right)\right\|_{W_{0}^{1, p}(\Omega)}^{q}\left(\left\|\varphi_{\varepsilon}\left(G_{k}(u)\right)\right\|_{W_{0}^{1, p}(\Omega)}^{p^{*}\left(1-\frac{q}{p}\right)}+\varepsilon^{\sigma p^{*}\left(1-\frac{q}{p}\right)}\right) \\
& \quad+C\left\|f \chi_{\left\{|f|>a_{k}\right\}}\right\|_{L^{m}(\Omega)}\left\|\varphi_{\varepsilon}\left(G_{k}(u)\right)\right\|_{W_{0}^{1, p}(\Omega)}^{\frac{p^{*}}{m^{\prime}}} \\
& \quad+\frac{1}{\varepsilon^{p(1-\sigma)+1}} \frac{\alpha_{0}}{\sqrt{h(k)}}\|g\|_{L^{1}(\Omega)}+\frac{1}{\varepsilon^{p(1-\sigma)}}\left\|g \chi_{\left\{|g|>b_{k}\right\}}\right\|_{L^{1}(\Omega)},
\end{aligned}
$$

where both $a_{k}=\frac{\alpha_{0}}{2} h(k)$ and $b_{k}=\frac{\alpha_{0}^{3}}{4} \sqrt{h(k)}$ tend to infinity as $k$ tends to infinity.

Then we use Young's inequality to obtain

$$
\begin{aligned}
\frac{\alpha}{2}\left\|\varphi_{\varepsilon}\left(G_{k}(u)\right)\right\|_{W_{0}^{1, p}(\Omega)}^{p} & \leq \gamma C\left\|\varphi_{\varepsilon}\left(G_{k}(u)\right)\right\|_{W_{0}^{1, p}(\Omega)}^{q+p^{*}\left(1-\frac{q}{p}\right)} \\
& +C \gamma \varepsilon^{\sigma\left(q+p^{*}\left(1-\frac{q}{p}\right)\right)}+\alpha^{-\frac{p^{*}}{p m^{\prime}-p^{*}}}\left\|f \chi_{\left\{|f|>a_{k}\right\}}\right\|_{L^{m}(\Omega)}^{\frac{p m^{\prime}}{p m^{\prime}-p^{*}}} \\
& +\frac{1}{\varepsilon^{p(1-\sigma)+1}} \frac{\alpha_{0}}{\sqrt{h(k)}}\|g\|_{L^{1}(\Omega)}+\frac{1}{\varepsilon^{p(1-\sigma)}}\left\|g \chi_{\left\{|g|>b_{k}\right\}}\right\|_{L^{1}(\Omega)} .
\end{aligned}
$$

If we set now

$$
Y_{k}=\left\|\varphi_{\varepsilon}\left(G_{k}(u)\right)\right\|_{W_{0}^{1, p}(\Omega)},
$$

we obtain, for some constants $C_{1}, C_{2}>0$,

$$
\begin{aligned}
\alpha C_{1} Y_{k}^{p}-\gamma C_{2} Y_{k}^{q+p^{*}\left(1-\frac{q}{p}\right)} \leq & \gamma \varepsilon^{\sigma\left(q+p^{*}\left(1-\frac{q}{p}\right)\right)}+\alpha^{-\frac{p^{*}}{p m^{\prime}-p^{*}}}\left\|f \chi_{\left\{|f|>a_{k}\right\}}\right\|_{L^{m}(\Omega)}^{\frac{p m^{\prime}}{p m^{\prime}-p^{*}}} \\
& +\frac{1}{\varepsilon^{p(1-\sigma)+1}} \frac{\alpha_{0}}{\sqrt{h(k)}}\|g\|_{L^{1}(\Omega)} \\
& +\frac{1}{\varepsilon^{p(1-\sigma)}}\left\|g \chi_{\left\{|g|>b_{k}\right\}}\right\|_{L^{1}(\Omega)},
\end{aligned}
$$

which we can rewrite, as in Theorem 3.1, as

$$
F\left(Y_{k}\right) \leq M_{\varepsilon, k}
$$


where

$$
F(Y)=\alpha C_{1} Y^{p}-\gamma C_{2} Y^{q+p^{*}\left(1-\frac{q}{p}\right)},
$$

and

$$
\begin{aligned}
M_{\varepsilon, k}= & \gamma \varepsilon^{\sigma\left(q+p^{*}\left(1-\frac{q}{p}\right)\right)}+\alpha^{-\frac{p^{*}}{p m^{\prime}-p^{*}}}\left\|f \chi_{\left\{|f|>a_{k}\right\}}\right\|_{L^{m}(\Omega)}^{\frac{p m^{\prime}}{p m^{\prime}-p^{*}}} \\
& +\frac{1}{\varepsilon^{p(1-\sigma)+1}} \frac{\alpha_{0}}{\sqrt{h(k)}}\|g\|_{L^{1}(\Omega)}+\frac{1}{\varepsilon^{p(1-\sigma)}}\left\|g \chi_{\left\{|g|>b_{k}\right\}}\right\|_{L^{1}(\Omega)},
\end{aligned}
$$

being $a_{k}=\frac{\alpha_{0}}{2} h(k)$ and $b_{k}=\frac{\alpha_{0}^{3}}{4} \sqrt{h(k)}$.

Note that, as in Theorem 3.1, the function $F$ is concave and has a maximum $F^{*}$ which can be computed, obtaining, for a constant $C^{*}$ which only depends on $p$, $q, N$ :

$$
F^{*}:=\max F(Y)=C^{*} \alpha^{\frac{N-q}{p-q}} \gamma^{-\frac{N-p}{p-q}} .
$$

Again we distinguish between two cases:

(i) if $\alpha_{0}=0$, then $M_{\varepsilon, k}$ does not depend on $k$ and becomes

$$
M_{\varepsilon}=\gamma \varepsilon^{\sigma\left(q+p^{*}\left(1-\frac{q}{p}\right)\right)}+\alpha^{-\frac{p^{*}}{p m^{\prime}-p^{*}}}\|f\|_{L^{m}(\Omega)}^{\frac{p m^{\prime}}{p m^{\prime}-p^{*}}}+\frac{1}{\varepsilon^{p(1-\sigma)}}\|g\|_{L^{1}(\Omega)} .
$$

We choose now $\varepsilon=\varepsilon_{0}$ as the minimum point of $M_{\varepsilon}$. After some computations that we skip and using the value of $\sigma$, it turns out that $\varepsilon_{0} \simeq\left(\frac{\|g\|_{L^{1}(\Omega)}}{\gamma}\right)^{\frac{1}{q+1}}$ and

$$
M_{0}=M_{\varepsilon_{0}}=\min _{\varepsilon} M_{\varepsilon}=\alpha^{-\frac{p^{*}}{p m^{\prime}-p^{*}}}\|f\|_{L^{m}(\Omega)}^{\frac{p m^{\prime}}{p m^{\prime}-p^{*}}}+\kappa_{0}\|g\|_{L^{1}(\Omega)}^{1-\theta} \gamma^{\theta}
$$

being $\theta=\frac{p(1-\sigma)}{q+1}>0$ and $\kappa_{0}>0$ a number depending only on $p, q, N$. Inequality (4.12) then becomes

$$
F\left(Y_{k}\right) \leq M_{0},
$$

where $Y_{k}$ is defined in (4.11) with $\varepsilon=\varepsilon_{0}$. We use now assumption (4.3) which is nothing but

$$
M_{0}<F^{*}=\max F(Y) .
$$

Then, with the same continuity argument used in Theorem 3.1, we end up with the estimate $Y_{k} \leq Z^{-}\left(M_{0}\right)$ for every $k \geq 0$, where $Z^{-}\left(M_{0}\right)$ is the first root of $F(Y)=M_{0}$. For $k=0$ this gives the desired estimate for $\varphi_{\varepsilon_{0}}(u)$ in $W_{0}^{1, p}(\Omega)$, i.e. for $\int_{0}^{u}\left(\varepsilon_{0}+|\xi|\right)^{\sigma-1} d \xi$, where $\varepsilon_{0}$ is the minimum point of $M_{\varepsilon}$. Since $\varepsilon_{0}$ only depends on $\|g\|_{L^{1}(\Omega)}$, we deduce an estimate on $(1+|u|)^{\sigma-1} u$ in $W_{0}^{1, p}(\Omega)$ depending on $\|f\|_{L^{m}(\Omega)},\|g\|_{L^{1}(\Omega)}$ and of course on $p, N, q$. 
(ii) if $\alpha_{0}>0$, we write $\varepsilon=\varepsilon_{1}+\varepsilon_{2}$ and we have

$$
\begin{aligned}
M_{\varepsilon, k} \leq & \alpha^{-\frac{p^{*}}{p m^{\prime}-p^{*}}}\left\|f \chi_{\left\{|f|>a_{k}\right\}}\right\|_{L^{m}(\Omega)}^{\frac{p m^{\prime}}{p m^{\prime}-p^{*}}} \\
& +C \gamma \varepsilon_{1}^{\sigma\left(q+p^{*}\left(1-\frac{q}{p}\right)\right)}+\frac{1}{\varepsilon_{1}^{p(1-\sigma)+1}} \frac{\alpha_{0}}{\sqrt{h(k)}}\|g\|_{L^{1}(\Omega)} \\
& +C \gamma \varepsilon_{2}^{\sigma\left(q+p^{*}\left(1-\frac{q}{p}\right)\right)}+\frac{1}{\varepsilon_{2}^{p(1-\sigma)}}\left\|g \chi_{\left\{|g|>b_{k}\right\}}\right\|_{L^{1}(\Omega)} .
\end{aligned}
$$

Now we choose $\varepsilon_{1}$ as the value which minimizes the second line and $\varepsilon_{2}$ as the one which minimizes the third line. In that way we find

$$
\begin{aligned}
M_{\varepsilon_{1}+\varepsilon_{2}, k} \leq & \alpha^{-\frac{p^{*}}{p m^{\prime}-p^{*}}}\left\|f \chi_{\left\{|f|>a_{k}\right\}}\right\|_{L^{m}(\Omega)}^{\frac{p m^{\prime}}{p m^{\prime}-p^{*}}} \\
& +c_{1} \gamma^{\theta_{1}}\left(\frac{\alpha_{0}}{\sqrt{h(k)}}\|g\|_{L^{1}(\Omega)}\right)^{1-\theta_{1}} \\
& +c_{2} \gamma^{\theta_{2}}\left\|g \chi_{\left\{|g|>b_{k}\right\}}\right\|_{L^{1}(\Omega)}^{1-\theta_{2}}
\end{aligned}
$$

where $c_{1}, c_{2}$ are constants depending only on $p, q, N$ and $0<\theta_{1}, \theta_{2}<1$ (the computations show that $\theta_{1}=\frac{p(1-\sigma)+1}{q+2}$ and $\theta_{2}=\frac{p(1-\sigma)}{q+1}$, but the precise values of $\theta_{1}$ and $\theta_{2}$ are inessential in the following).

Note that $\varepsilon_{1}, \varepsilon_{2}$ depend on $k$, and actually we have

$$
\varepsilon_{1} \simeq\left(\frac{\alpha_{0}}{\sqrt{h(k)}} \frac{\|g\|_{L^{1}(\Omega)}}{\gamma}\right)^{\frac{1}{q+2}}, \quad \varepsilon_{2} \simeq\left(\frac{\left\|g \chi_{\left\{|g|>b_{k}\right\}}\right\|_{L^{1}(\Omega)}}{\gamma}\right)^{\frac{1}{q+1}}
$$

We fix henceforth the value of $\varepsilon$ and we denote it by $\varepsilon_{k}=\varepsilon_{1}+\varepsilon_{2}$. In particular, observe that $\varepsilon_{k}$ tends to 0 as $k$ tends to $\infty$. After such a choice, inequality (4.12) only depends on $k$ and becomes

$$
F\left(Y_{k}\right) \leq \tilde{M}_{k}
$$

where

$$
\begin{aligned}
\tilde{M}_{k}= & \alpha^{-\frac{p^{*}}{p m^{\prime}-p^{*}}}\left\|f \chi_{\left\{f>a_{k}\right\}}\right\|_{L^{m}(\Omega)}^{\frac{p m^{\prime}}{p m^{\prime}-p^{*}}} \\
& +c_{1} \gamma^{\theta_{1}}\left(\frac{\alpha_{0}}{\sqrt{h(k)}}\|g\|_{L^{1}(\Omega)}\right)^{1-\theta_{1}} \\
& +c_{2} \gamma^{\theta_{2}}\left\||g| \chi_{\left\{|g|>b_{k}\right\}}\right\|_{L^{1}(\Omega)}^{1-\theta_{2}} .
\end{aligned}
$$


Using that $a_{k}$ and $b_{k}$ tend to infinity as $k$ tends to infinity, $\tilde{M}_{k}$ will be smaller than $F^{*}$ for $k$ sufficiently large. Since we also have that $\varepsilon_{k}$ tends to zero when $k$ goes to infinity, we are allowed to define

$$
k^{*}=\inf \left\{k>0: \varepsilon_{k} \leq 1, \tilde{M}_{k}<F^{*}\right\},
$$

and we can apply the same argument as in Theorem 3.1 for $k>k^{*}$. We obtain in this way an estimate for $\left\|\varphi_{\varepsilon_{k}}\left(G_{k}(u)\right)\right\|_{W_{0}^{1, p}(\Omega)}$ for every $k>k^{*}$. Since the choice of $k^{*}$ implies that $\varepsilon_{k} \leq 1$, and since by definition of $\varphi_{\varepsilon}$ we have $\left\|\varphi_{\varepsilon}\left(G_{k}(u)\right)\right\|_{W_{0}^{1, p}(\Omega)} \geq$ $\left\|\varphi_{1}\left(G_{k}(u)\right)\right\|_{W_{0}^{1, p}(\Omega)}$ for any $\varepsilon \leq 1$, we have obtained an estimate for $\varphi_{1}\left(G_{k}(u)\right)$ in $W_{0}^{1, p}(\Omega)$ for every $k>k^{*}$. In particular, since

$$
\begin{aligned}
\left\|\varphi_{1}\left(G_{k}(u)\right)\right\|_{W_{0}^{1, p}(\Omega)}^{p} & =\int_{\Omega}\left|D G_{k}(u)\right|^{p}\left(1+\left|G_{k}(u)\right|\right)^{p(\sigma-1)}\left(\frac{\left|G_{k}(u)\right|}{\alpha_{0}+\left|G_{k}(u)\right|}\right) d x \\
& \geq \frac{1}{\alpha_{0}+1} \int_{\Omega}\left|D G_{k+1}(u)\right|^{p}\left(2+\left|G_{k+1}(u)\right|\right)^{p(\sigma-1)} d x,
\end{aligned}
$$

we deduce an estimate on $\left\|\left(1+\left|G_{k}(u)\right|\right)^{\sigma-1} G_{k}(u)\right\|_{W_{0}^{1, p}(\Omega)}$ for $k>k^{*}+1$, more precisely we get

$$
\left\|\left(1+\left|G_{k}(u)\right|\right)^{\sigma-1} G_{k}(u)\right\|_{W_{0}^{1, p}(\Omega)} \leq C Z^{-}\left(\tilde{M}_{k}\right),
$$

where $Z^{-}\left(\tilde{M}_{k}\right)$ is the first root of the equation $F(Y)=\tilde{M}_{k}$.

Finally, using the truncation function, we complete the estimate when $|u|$ is small as in Theorem 3.1, obtaining the first estimate in (4.5).

The estimate on $(1+|u|)^{\sigma-1} u$ in $W_{0}^{1, p}(\Omega)$, with $\sigma<1$, yields in turn the estimate for $|D u|^{p-1}$ in some space $L^{s}(\Omega)$ with $s$ depending on $\sigma$ : this is a classical result proved by L. Boccardo and T. Gallouët in [12] (at least when $p>2-\frac{1}{N}$, but the proof can be adapted for all $p>1$ ). Indeed, when specialized to our case (i.e. for the precise value of $\sigma$ ), one has

$$
\int_{\Omega}|\nabla u|^{N(q-(p-1))} d x \leq\left\|(1+|u|)^{\sigma-1} u\right\|_{W_{0}^{1, p}(\Omega)}^{N(q-(p-1))}\left\|(1+|u|)^{\sigma}\right\|_{L^{p^{*}(\Omega)}}^{p^{*}\left(1-\frac{N(q-(p-1))}{p}\right)},
$$

which gives an estimate for $|D u|^{p-1}$ in $L^{\frac{N(q-(p-1))}{p-1}}(\Omega)$.

Remark 4.6. When $\alpha_{0}>0$, thanks to estimate (4.16), we deduce, as in Remark 3.5 , that $\left\|\left|G_{k}(u)\right|^{\sigma}\right\|_{L^{p^{*}}(\Omega)}$ tends to zero when $k$ tends to infinity, uniformly when $f$ and $g$ vary in sets that are bounded and equi-integrable in $L^{m}(\Omega)$ and $L^{1}(\Omega)$ respectively. Then (4.17) (used with $G_{k}(u)$ ) implies that

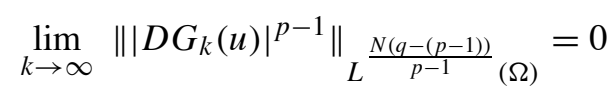

uniformly when $f$ and $g$ vary in such sets. 
When $\alpha_{0}=0$, a similar equi-integrability result can be proved if $\|u\|_{L^{1}(\Omega)}$ remains bounded. This can be obtained by using a variant of the above proof in the same way as explained in Remark 3.6.

We apply Theorem 4.3 to extend the existence result of Theorem 3.7 to this range of $q$ and $m$.

Theorem 4.7. Assume (2.2)-(2.7) and (3.34), (3.35), with $\frac{N(p-1)}{N-1}<q<p-1+$ $\frac{p}{N}$, and that either

(i) $\alpha_{0}>0$,

or

(ii) $\alpha_{0}=0$ and (4.3) holds true.

Then there exists $u$ satisfying (4.4) which is a renormalized solution of (2.1) in the sense of Definition 4.1. Moreover, $|D u|^{p-1} \in L^{\frac{N(q-(p-1))}{p-1}}(\Omega)$.

Proof. As in the proof of Theorem 3.7, we consider the sequence of approximating solutions $u_{n} \in W_{0}^{1, p}(\Omega)$ of problem (3.36). Since $u_{n} \in W_{0}^{1, p}(\Omega)$, weak solutions are also renormalized solution in the sense of Definition 4.1. Applying Theorem 4.3 we have that $\left(1+\left|u_{n}\right|\right)^{\sigma-1} u_{n}$ is bounded in $W_{0}^{1, p}(\Omega)$, which implies that, for fixed $k>0$, the truncation $T_{k}\left(u_{n}\right)$ is bounded in $W_{0}^{1, p}(\Omega)$. Moreover $\left|D u_{n}\right|^{q}$ is bounded in $L^{\frac{N(q-(p-1))}{q}}(\Omega)$ (note that since $q>\frac{N(p-1)}{N-1}$, we have $\frac{N(q-(p-1))}{q}>1$ ). Therefore, using (3.35), we deduce that $T_{n}\left(H\left(x, u_{n}, D u_{n}\right)\right)$ is bounded in $L^{\tau}(\Omega)$ with $\tau=\min \left(\frac{N(q-(p-1))}{q}, m\right)$. Since the right-hand side of (3.36) is bounded in $L^{1}(\Omega)$, again we can use compactness results in the literature (firstly due to [12], see also the proofs in $[9,39])$ to deduce that, up to extracting a subsequence, $u_{n}$ converges almost everywhere to some $u$ such that $(1+|u|)^{\sigma-1} u \in W_{0}^{1, p}(\Omega)$, and $D u_{n}$ converges to $D u$ almost everywhere in $\Omega$ with $|D u|^{p-1} \in L^{\frac{N(q-(p-1))}{p-1}}(\Omega)$. As a consequence, the term $T_{n}\left(H\left(x, u_{n}, D u_{n}\right)\right)$ strongly converges in $L^{1}(\Omega)$. Together with (3.34), this yields the compactness of $a_{0}\left(x, u_{n}\right)$ in $L^{1}(\Omega)$. Moreover, since the lower order terms are compact in $L^{1}(\Omega)$, we deduce the compactness of $T_{k}\left(u_{n}\right)$ in $W_{0}^{1, p}(\Omega)$ for any $k>0$ (see [32], [38]). Thanks to this latter fact, it is possible to pass to the limit in the renormalized formulation, proving that $u$ satisfies (4.1).

Remark 4.8. Since $|D u|^{p-1} \in L^{\frac{N(q-(p-1))}{p-1}}(\Omega)$, then $|D u|^{q-(p-1)} \in L^{N}(\Omega)$. As a consequence, we can write

$$
|D u|^{q}=B(x)|D u|^{p-1} \quad \text { with } B(x) \in L^{N}(\Omega) .
$$

In this case one can look at problem (2.1) as a problem where the Hamiltonian term has "linear" growth (or rather, $p-1$-growth, referring to the $p$-Laplace operator) weighted with a $L^{N}$ potential. This sort of "linearization" approach is very useful 
for further results (see e.g. the uniqueness results in [5,40], or the bootstrap result of the next Theorem 4.9).

Thanks to Remark 4.8, it is easier in this case to prove the analogue of the bootstrap regularity result of Theorem 3.8 .

Theorem 4.9. Assume (2.2), (2.3), (2.5), (2.6), (2.7) with $\frac{N(p-1)}{N-1}<q<p-1+\frac{p}{N}$. Assume in addition that $f$ belongs to $L^{s}(\Omega)$, with $s>\frac{N(q-(p-1))}{q}$ and $g$ belongs to $L^{t}(\Omega)$, with $t \geq 1$.

Let $u$ satisfy (4.1) and (4.4). Then we have

(i) If $s>\frac{N}{p}$ and $t>\frac{N}{p}$, then $u \in L^{\infty}(\Omega)$ and

$$
\|u\|_{L^{\infty}(\Omega)} \leq M .
$$

(ii) If $s=t=\frac{N}{p}$, then $u \in L^{d}(\Omega)$ for any $d<\infty$ and

$$
\|u\|_{L^{d}(\Omega)} \leq M .
$$

(iii) If $\left(p^{*}\right)^{\prime}<s<\frac{N}{p}$ and $t \geq \frac{N s(p-1)}{p(N-s)}$, then $|u|^{\tau} \in W_{0}^{1, p}(\Omega), u \in L^{\left(s^{*}(p-1)\right)^{*}}(\Omega)$ and

$$
\left\||u|^{\tau}\right\|_{W_{0}^{1, p}(\Omega)}+\|u\|_{L^{\left(s^{*}(p-1)\right)^{*}(\Omega)}} \leq M, \quad \tau=\frac{s(N-p)(p-1)}{p(N-s p)} .
$$

(iv) If $\frac{N(q-(p-1))}{q}<s \leq\left(p^{*}\right)^{\prime}$ and $t \geq 1$, then $(1+|u|)^{\tau} \in W_{0}^{1, p}(\Omega),|D u|^{p-1} \in$ $L^{s^{*}}(\Omega)$ and

$$
\left\|(1+|u|)^{\tau-1} u\right\|_{W_{0}^{1, p}(\Omega)}+\left\||D u|^{p-1}\right\|_{L^{s^{*}}(\Omega)} \leq M, \quad \tau=\frac{s(N-p)(p-1)}{p(N-s p)} .
$$

In (4.18)-(4.21), the constant $M$ depends on $p, q, s, t, N, \alpha_{0}, \alpha, \gamma,|\Omega|, d$ (in case (4.19)) and on $\|f\|_{L^{s}(\Omega)}$ and $\|g\|_{L^{t}(\Omega)}$; in the sole case (iv), $M$ depends also on the equi-integrability of $g$ in $L^{1}(\Omega)$. Moreover, when $\alpha_{0}=0, M$ depends on $\|u\|_{L^{1}(\Omega)}$ as well.

Proof. We only sketch the main arguments. Using (2.2), (2.5) and (2.6), one obtains from the renormalized equation (4.1):

$$
\begin{aligned}
\alpha \int_{\Omega}\left|D G_{k}(u)\right|^{p} \Phi^{\prime}\left(G_{k}(u)\right) d x \leq & \gamma \int_{\Omega}\left|D G_{k}(u)\right|^{q} \Phi\left(G_{k}(u)\right) d x \\
& +\int_{\Omega}|f|\left|\Phi\left(G_{k}(u)\right)\right| d x \\
& +\int_{\Omega}|g|\left|\Phi^{\prime}\left(G_{k}(u)\right)\right| d x .
\end{aligned}
$$


However, in order that the function $\Phi$ be justified in (4.22), one needs, in virtue of (4.4), that

$$
0 \leq \Phi^{\prime}(s) \leq L(1+s)^{(\sigma-1) p}
$$

for some constant $L$, where $\sigma$ is defined in (4.4). We also require that

$$
\frac{\Phi(s)}{\Phi^{\prime}(s)^{\frac{1}{p^{\prime}}}} \leq C \int_{0}^{s} \Phi^{\prime}(\xi)^{\frac{1}{p}} d \xi
$$

and so we have

$$
\begin{aligned}
& \int_{\Omega}\left|D G_{k}(u)\right|^{q} \Phi\left(G_{k}(u)\right) d x \\
& \leq C \int_{\Omega}\left|D G_{k}(u)\right|^{q-(p-1)}\left|D G_{k}(u)\right|^{p-1} \Phi^{\prime}\left(G_{k}(u)\right)^{\frac{1}{p^{\prime}}}\left(\int_{0}^{G_{k}(u)} \Phi^{\prime}(\xi)^{\frac{1}{p}} d \xi\right) d x .
\end{aligned}
$$

From Hölder inequality with exponents $N, p^{\prime}, p^{*}$, and Sobolev inequality, we get

$$
\begin{aligned}
& \int_{\Omega}\left|D G_{k}(u)\right|^{q} \Phi\left(G_{k}(u)\right) d x \\
& \leq C\left\|\left|D G_{k}(u)\right|^{q-(p-1)}\right\|_{L^{N}(\Omega)}\left(\int_{\Omega}\left|D G_{k}(u)\right|^{p} \Phi^{\prime}\left(G_{k}(u)\right) d x\right) .
\end{aligned}
$$

Choose now $k_{0}$ such that

$$
\gamma C\left\|\left|D G_{k}(u)\right|^{q-(p-1)}\right\|_{L^{N}(\Omega)}<\frac{\alpha}{2} \quad \forall k \geq k_{0} .
$$

Note that this makes sense by means of Remark 4.8. Then (4.22) implies

$$
\frac{\alpha}{2} \int_{\Omega}\left|D G_{k}(u)\right|^{p} \Phi^{\prime}\left(G_{k}(u)\right) d x \leq \int_{\Omega}|f|\left|\Phi\left(G_{k}(u)\right)\right| d x+\int_{\Omega}|g|\left|\Phi^{\prime}\left(G_{k}(u)\right)\right| d x .
$$

In order to obtain the regularity and the estimates in the statement we only need to choose a suitable $\Phi$ here. For instance, (4.20) (and then (4.19)) follow by choosing

$$
\Phi(s)=\int_{0}^{s}(1+|\xi|)^{p(\sigma-1)}\left|T_{n}(\xi)\right|^{p(\tau-\sigma)} d \xi
$$

where $\tau>\sigma$ is chosen so that $\tau p^{*}=((\tau-1) p+1) s^{\prime}$ (as in Theorem 3.8). One can easily verify that $\Phi$ satisfies (4.23)-(4.24). Then, from (4.26), one can conclude reasoning as in Theorem 3.8 .

Finally, the estimates found will depend on $\|f\|_{L^{s}(\Omega)},\|g\|_{L^{t}(\Omega)}$ and on the number $k_{0}$ defined in (4.25). Thanks to Remark 4.6, the number $k_{0}$ depends on 
the bound and the equi-integrability of $f$ and $g$ in $L^{\frac{N(q-(p-1))}{q}}(\Omega)$ and in $L^{1}(\Omega)$, respectively, and, only when $\alpha_{0}=0$, on $\|u\|_{L^{1}(\Omega)}$ as well. As in Theorem 3.8, this explains the statement concerning the dependence of the constant $M$ in estimates (4.18)-(4.21). Observe that in cases (i)-(iii) one has $s>\frac{N(q-(p-1))}{q}$ and $t>1$, and so a bound on $f$ and $g$ in $L^{s}(\Omega)$ and $L^{t}(\Omega)$ is enough to imply both a bound and the equi-integrability of $f$ and $g$ required to estimate $k_{0}$; on the other hand, in case (iv) we may possibly have $t=1$ and we need to require explicitly the equi-integrability of $g$ in $L^{1}(\Omega)$.

Let us conclude this section by looking briefly at the case $q=\frac{N(p-1)}{N-1}$. Observe that this limiting value of $q$ corresponds, through (2.7), to $m=1$ and $r=1$. Nevertheless, it is not possible to consider general data in $L^{1}(\Omega)$ for this situation, as explained by the following example.

Example 4.10. Assume that $f \in L^{1}(\Omega), f \geq 0$, and that there exists a renormalized solution $u$ of the problem

$$
\begin{cases}-\Delta_{p} u=|D u|^{\frac{N(p-1)}{N-1}}+f & \text { in } \Omega, \\ u=0 & \text { on } \partial \Omega .\end{cases}
$$

In particular, we have that $|D u| \in L^{\frac{N(p-1)}{N-1}}(\Omega)$ (for simplicity let assume here that $p \geq 2-\frac{1}{N}$, so that $\left.\frac{N(p-1)}{N-1} \geq 1\right)$. Then, by Sobolev embedding, $u \in L^{\frac{N(p-1)}{N-p}}(\Omega)$. By comparison, the (unique renormalized) solution of

$$
\begin{cases}-\Delta_{p} v=f & \text { in } \Omega, \\ v=0 & \text { on } \partial \Omega,\end{cases}
$$

satisfies $0 \leq v \leq u$, hence $v$ also belongs to $L^{\frac{N(p-1)}{N-p}}(\Omega)$. However, it is well-known that this is not true if one takes a general function $f \in L^{1}(\Omega)$.

In view of the above example, the assumption $f \in L^{1}(\Omega)$ is not enough to have existence of solutions when $q=\frac{N(p-1)}{N-1}$. On the other hand, using our previous results, we can prove estimates assuming that

$$
f \in L^{\hat{m}}(\Omega) \quad \text { for some } \hat{m}>1 .
$$

Indeed, if $H(x, s, \xi)$ satisfies the growth condition (3.35), we have, thanks to Young's inequality,

$$
|H(x, s, \xi)| \leq \gamma|\xi|^{\frac{N(p-1)}{N-1}}+f(x) \leq \tilde{\gamma}|\xi|^{\tilde{q}}+\tilde{f}(x)
$$

for any $\tilde{q}$ such that $\tilde{q}>\frac{N(p-1)}{N-1}$ and $\hat{m}>\frac{N(\tilde{q}-(p-1))}{\tilde{q}}$. In this way we can apply the results in Theorem 4.3 and Theorem 4.9; since any bounded sequence in $L^{\hat{m}}(\Omega)$ 
is equi-integrable in $L^{\frac{N(\tilde{q}-(p-1))}{\tilde{q}}}(\Omega)$, we obtain an a priori bound for $u$ depending only on $\|f\|_{L^{\hat{m}}(\Omega)}$. This allows us to get estimates and the existence of a solution. Of course, in case $\alpha_{0}=0$ some size condition on $\|f\|_{L^{m}(\Omega)}$ and $\|g\|_{L^{1}(\Omega)}$ are still needed; in this approach it would sound as in (4.3) for the $\tilde{q}$ used above. For simplicity, in the next statement with $\alpha_{0}=0$ we only give a vague form of this condition by saying that $\|f\|_{L^{m}(\Omega)}$ and $\|g\|_{L^{1}(\Omega)}$ should be taken sufficiently small.

Theorem 4.11. Assume (2.2)-(2.6), with $q=\frac{N(p-1)}{N-1}, m>1, r=1$, and that either

(i) $\alpha_{0}>0$,

or

(ii) $\alpha_{0}=0$ and $\|f\|_{L^{m}(\Omega)}$ and $\|g\|_{L^{1}(\Omega)}$ are small enough.

Let $u$ be a renormalized solution of (2.1), in the sense of Definition 4.1, which satisfies the regularity condition

$$
(1+|u|)^{\sigma-1} u \in W_{0}^{1, p}(\Omega) \quad \text { for some } \sigma>\frac{p-1}{p} .
$$

Then we have, for $\tau=\frac{m(N-p)(p-1)}{p(N-m p)}$

$$
\left\|(1+|u|)^{\tau-1} u\right\|_{W_{0}^{1, p}(\Omega)}+\left\||D u|^{p-1}\right\|_{L^{m^{*}}(\Omega)} \leq M,
$$

where $M$ depends on $p, q, N, \alpha_{0}, \alpha, \gamma,|\Omega|$ and remains bounded when $f$ and $g$ vary in sets which are, respectively, bounded in $L^{m}(\Omega)$ and equi-integrable in $L^{1}(\Omega)$. Moreover, under the above assumptions together with (3.34) and (3.35), there exists at least one renormalized solution $u$ of (2.1) (in the sense of Definition 4.1) satisfying (4.29).

Remark 4.12. As already mentioned, the result in Theorem 4.11 is not optimal: indeed, a sharp assumption on $f$ should consider some interpolation space between $L^{1}$ and $L^{m}(\Omega)$ for any $m>1$. By looking a bit at the radial case, it seems possible to conjecture that an optimal assumption could be the Orlicz space

$$
f \in L^{1}\left((\log L)^{N-1}\right) .
$$

However, a priori estimates and eventually existence of solutions under this condition is, to our knowledge, an open problem, and certainly this borderline case should deserve further investigation.

\section{The case $q<\frac{N(p-1)}{N-1}$}

In this section we assume (2.2)-(2.6) with $r=1, m=1$ and $p-1<q<\frac{N(p-1)}{N-1}$. In this range the growth of the Hamiltonian allows us to consider data in $L^{1}(\Omega)$, and 
even more generally, source terms which are bounded Radon measures. Therefore, we consider the problem

$$
\begin{cases}-\operatorname{div}(a(x, u, D u))+a_{0}(x, u)=H(x, u, D u)+\mu & \text { in } \Omega \\ u=0 & \text { on } \partial \Omega\end{cases}
$$

where $\mu$ is a bounded Radon measure on $\Omega$.

We recall (see $[13,28]$ ) that any bounded Radon measure $\mu$ admits a unique decomposition as $\mu=\mu_{0}+\mu_{s}$, where $\mu_{s}$ is concentrated on a set of null $p$ capacity (the $p$-capacity is the usual notion of capacity defined in $W_{0}^{1, p}(\Omega)$ ) and $\mu_{0} \in L^{1}(\Omega)+W^{-1, p^{\prime}}(\Omega)$. By saying that $\mu_{s}$ is concentrated on a set of null capacity we mean, precisely, that there exists a set $E$ which has $p$-capacity zero and such that

$$
\mu_{s}(B)=\mu_{s}(B \cap E)
$$

for any Borelian set $B$.

Remark 5.1. Observe that, writing $\mu_{0}=h+\operatorname{div}(H)$, with $h \in L^{1}(\Omega)$ and $H \in$ $L^{p^{\prime}}(\Omega)^{N}$, in (5.1) we can reduce $\mu$ to its singular part $\mu_{s}$ up to replacing $f$ with $f+|h|$ in assumption (2.6) and $g$ with $g+|H|^{p^{\prime}}$ in (2.2).

In order to study problem (5.1), we still adopt the framework of renormalized solutions, following the definition developed in [22] for the case of general measures as data. For more details concerning the origin and motivation of such definition, the role of capacity and fine properties of solutions and other related topics, we refer the reader to [22]. Here, without much more explanation, we make use of this setting in order to have proper statements of existence and a priori estimates similar to those given in the previous sections.

We define $\mathcal{T}_{0}^{1, p}(\Omega)$ as the set of all measurable functions $u: \Omega \rightarrow \mathbb{R}$ almost everywhere finite and such that the truncations $T_{k}(u)$ belong to $W_{0}^{1, p}(\Omega)$ for all $k>0$. For functions in $\mathcal{T}_{0}^{1, p}(\Omega)$, a notion of generalized gradient can be defined (see [6], [22]).

Definition 5.2. A function $u \in \mathcal{T}_{0}^{1, p}(\Omega)$ is a renormalized solution of (5.1) if it satisfies

$$
\begin{gathered}
\int_{\Omega} a(x, u, D u) D(S(u) \varphi) d x+\int_{\Omega} a_{0}(x, u) S(u) \varphi d x \\
=\int_{\Omega} H(x, u, D u) S(u) \varphi d x+\int_{\Omega} S(u) \varphi d \mu_{0},
\end{gathered}
$$

for any Lipschitz function $S$ having compact support and for any $\varphi \in W^{1, p}(\Omega) \cap$ 
$L^{\infty}(\Omega)$ such that $S(u) \varphi \in W_{0}^{1, p}(\Omega)$, and moreover

$$
\begin{aligned}
& \lim _{n \rightarrow \infty} \frac{1}{n} \int_{\{n<u<2 n\}} a(x, u, D u) D u \varphi d x=\int_{\Omega} \varphi d \mu_{s}^{+}, \\
& \lim _{n \rightarrow \infty} \frac{1}{n} \int_{\{-2 n<u<-n\}} a(x, u, D u) D u \varphi d x=\int_{\Omega} \varphi d \mu_{s}^{-},
\end{aligned}
$$

for every $\varphi \in C_{b}(\Omega)$, i.e. $\varphi$ continuous and bounded in $\Omega$.

If $r>1$, we also denote by $M^{r}(\Omega)$ the Marcinkiewicz space of order $r$ (also called in the literature weak Lebesgue space), with the norm given by

$$
\|u\|_{M^{r}(\Omega)}^{r}=\sup _{k>0}\left[k^{r} \operatorname{meas}\{x \in \Omega:|u(x)|>k\}\right] .
$$

Theorem 5.3. Assume (2.2), (2.3), (2.5), (2.6), with $r=1, m=1$ and $q<\frac{N(p-1)}{N-1}$. Assume further that either

(i) $\alpha_{0}=0$ and

$$
\alpha^{-\frac{1}{p}}\left(\|f\|_{L^{1}(\Omega)}+\|\mu\|_{\mathcal{M}_{b}(\Omega)}\right)+\|g\|_{L^{1}(\Omega)}^{\frac{1}{p^{\prime}}}<C^{*} \alpha^{\frac{q+1}{(q-(p-1)) p^{\prime}}} \gamma^{-\frac{p-1}{q-(p-1)}}
$$

where $C^{*}$ is a constant depending only on $p, q, N$ which will be defined in (5.12), or

(ii) $\alpha_{0}>0$ and

$$
\left\|\mu_{s}\right\|_{\mathcal{M}_{b}(\Omega)}<C^{*} \alpha^{\frac{q}{q-(p-1)}} \gamma^{-\frac{p-1}{q-(p-1)}} .
$$

Let $u$ be a renormalized solution of (5.1). Then we have

$$
\left\||u|^{p-1}\right\|_{M^{\frac{N}{N-p}}(\Omega)}+\left\||D u|^{p-1}\right\|_{M^{N-1}(\Omega)} \leq M
$$

where $M$ depends on $p, q, N, \alpha_{0}, \alpha, \gamma,|\Omega|$ and $f, g, \mu$. When $\alpha_{0}=0$ the constant $M$ depends on $f, g, \mu$ only through $\|f\|_{L^{1}(\Omega)},\|g\|_{L^{1}(\Omega)}$ and $\|\mu\|_{\mathcal{M}_{b}(\Omega)}$. When $\alpha_{0}>$ 0 , the constant $M$ does not depend only on $\|f\|_{L^{1}(\Omega)}$, $\|g\|_{L^{1}(\Omega)},\|\mu\|_{\mathcal{M}_{b}(\Omega)}$, but remains bounded when $f$ and $g$ vary in sets which are bounded and equi-integrable in $L^{1}(\Omega)$ and $\mu_{0}$ varies in a compact set of $L^{1}(\Omega)+W^{-1, p^{\prime}}(\Omega)$.

Remark 5.4. The content of Remarks 3.2 and 3.3 is also valid as far as Theorem 5.3 is concerned. The same holds for Remark 3.4 up to considering now nonnegative renormalized subsolutions. 
Proof. By standard renormalization arguments (see [22]), it is possible to consider in (5.2) test functions $S(u)$ such that $S^{\prime}$ has compact support and $S(0)=0$. With an approximation argument with compactly supported functions, and taking $\varphi=1$, one obtains the inequality

$$
\begin{array}{r}
\int_{\Omega} a(x, u, D u) D u S^{\prime}(u) d x+\int_{\Omega} a_{0}(x, u) S(u) d x \\
\quad \leq \int_{\Omega} H(x, u, D u) S(u) d x+\|S\|_{\infty}\|\mu\|_{\mathcal{M}_{b}(\Omega)} .
\end{array}
$$

We take here

$$
S(u)=T_{j}\left(G_{k}(u)\right) \frac{\left|T_{j}\left(G_{k}(u)\right)\right|}{\varepsilon+\left|T_{j}\left(G_{k}(u)\right)\right|},
$$

and then we get, thanks to (2.2), (2.5) and (2.6),

$$
\begin{aligned}
& \alpha \int_{\Omega}\left|D T_{j}\left(G_{k}(u)\right)\right|^{p} \frac{\left|T_{j}\left(G_{k}(u)\right)\right|}{\varepsilon+\left|T_{j}\left(G_{k}(u)\right)\right|} d x+\alpha_{0} \int_{\Omega} h(|u|) \frac{T_{j}\left(G_{k}(u)\right)^{2}}{\varepsilon+\left|T_{j}\left(G_{k}(u)\right)\right|} d x \\
& \quad \leq \gamma \int_{\Omega}|D u|^{q} \frac{T_{j}\left(G_{k}(u)\right)^{2}}{\varepsilon+\left|T_{j}\left(G_{k}(u)\right)\right|} d x+\int_{\Omega}|f| \frac{T_{j}\left(G_{k}(u)\right)^{2}}{\varepsilon+\left|T_{j}\left(G_{k}(u)\right)\right|} d x \\
& \quad+2 \int_{\Omega}|g| \frac{\left|T_{j}\left(G_{k}(u)\right)\right|}{\varepsilon+\left|T_{j}\left(G_{k}(u)\right)\right|} d x+j\|\mu\|_{\mathcal{M}_{b}(\Omega) .}
\end{aligned}
$$

Set

$$
\varphi_{\varepsilon}(s)=\int_{0}^{s}\left(\frac{|\xi|}{\varepsilon+|\xi|}\right)^{\frac{1}{p}} d \xi
$$

Then we have

$$
\begin{aligned}
\int_{\Omega}\left|D T_{j}\left(G_{k}(u)\right)\right|^{p} \frac{\left|T_{j}\left(G_{k}(u)\right)\right|}{\varepsilon+\left|T_{j}\left(G_{k}(u)\right)\right|} d x & =\int_{\Omega} \mid D\left(\left.\varphi_{\varepsilon}\left(T_{j}\left(G_{k}(u)\right)\right)\right|^{p} d x\right. \\
& =\int_{\Omega} \mid D T_{\varphi_{\varepsilon}(j)}\left(\left.\varphi_{\varepsilon}\left(G_{k}(u)\right)\right|^{p} d x,\right.
\end{aligned}
$$

where we used that $\varphi_{\varepsilon}$ is an odd increasing function. have

Moreover, using that $\frac{\left|G_{k}(u)\right|}{\varepsilon+\left|G_{k}(u)\right|} \geq \frac{\left|T_{j}\left(G_{k}(u)\right)\right|}{\varepsilon+\left|T_{j}\left(G_{k}(u)\right)\right|}$, and since $\frac{s^{2}}{\varphi_{\varepsilon}^{\prime}(s)^{q}(\varepsilon+|s|)} \leq|s|$, we

$$
\begin{aligned}
\int_{\Omega}|D u|^{q} \frac{T_{j}\left(G_{k}(u)\right)^{2}}{\varepsilon+\left|T_{j}\left(G_{k}(u)\right)\right|} d x & =\int_{\Omega} \frac{\left|D \varphi_{\varepsilon}\left(G_{k}(u)\right)\right|^{q}}{\varphi_{\varepsilon}^{\prime}\left(G_{k}(u)\right)^{q}} \frac{T_{j}\left(G_{k}(u)\right)^{2}}{\varepsilon+\left|T_{j}\left(G_{k}(u)\right)\right|} d x \\
& \leq \int_{\Omega} \frac{\left|D \varphi_{\varepsilon}\left(G_{k}(u)\right)\right|^{q}}{\varphi_{\varepsilon}^{\prime}\left(T_{j}\left(G_{k}(u)\right)\right)^{q}} \frac{T_{j}\left(G_{k}(u)\right)^{2}}{\varepsilon+\left|T_{j}\left(G_{k}(u)\right)\right|} d x \\
& \leq j \int_{\Omega}\left|D \varphi_{\varepsilon}\left(G_{k}(u)\right)\right|^{q} d x .
\end{aligned}
$$


As we estimated in previous sections, we have

$$
\begin{aligned}
& \int_{\Omega}|f| \frac{T_{j}\left(G_{k}(u)\right)^{2}}{\varepsilon+\left|T_{j}\left(G_{k}(u)\right)\right|} d x+2 \int_{\Omega}|g| \frac{\left|T_{j}\left(G_{k}(u)\right)\right|}{\varepsilon+\left|T_{j}\left(G_{k}(u)\right)\right|} d x \\
& \leq \alpha_{0} \int_{\Omega} h(|u|) \frac{T_{j}\left(G_{k}(u)\right)^{2}}{\varepsilon+\left|T_{j}\left(G_{k}(u)\right)\right|} d x+\int_{\left\{|f|>\frac{\alpha_{0}}{2} h(k)\right\}}|f| \frac{T_{j}\left(G_{k}(u)\right)^{2}}{\varepsilon+\left|T_{j}\left(G_{k}(u)\right)\right|} d x \\
& \quad+2 \int_{\left\{|g|>\frac{\alpha_{0}}{4} h(|u|)\left|T_{j}\left(G_{k}(u)\right)\right|\right\}} \mid \frac{\left|T_{j}\left(G_{k}(u)\right)\right|}{\varepsilon+\left|T_{j}\left(G_{k}(u)\right)\right|} d x \\
& \leq \alpha_{0} h(|u|) \frac{T_{j}\left(G_{k}(u)\right)^{2}}{\varepsilon+\left|T_{j}\left(G_{k}(u)\right)\right|} d x+j\left\|f \chi_{\left\{|f|>\frac{\alpha_{0}}{2} h(k)\right\}}\right\|_{L^{1}(\Omega)} \\
& \left.\quad+2 \frac{\delta_{k}}{\varepsilon}\|g\|_{L^{1}(\Omega)}+2|| g \chi_{\left\{|g|>\frac{\alpha_{0}}{4}\right.} h(k) \delta_{k}\right\} \\
& L_{L^{1}(\Omega)},
\end{aligned}
$$

so that, choosing for instance $\delta_{k}=\frac{\alpha_{0}}{\sqrt{h(k)}}$, we get, collecting $(5.7),(5.8),(5.9)$ and (5.10),

$$
\begin{aligned}
& \alpha \int_{\Omega} \mid D T_{\varphi_{\varepsilon}(j)}\left(\left.\varphi_{\varepsilon}\left(G_{k}(u)\right)\right|^{p} d x\right. \\
& \leq j\left(\gamma \int_{\Omega}\left|D \varphi_{\varepsilon}\left(G_{k}(u)\right)\right|^{q} d x+\left\|f \chi_{\left\{|f|>a_{k}\right\}}\right\|_{L^{1}(\Omega)}+\|\mu\|_{\mathcal{M}_{b}(\Omega)}\right) \\
& \quad+\frac{2 \alpha_{0}}{\varepsilon \sqrt{h(k)}}\|g\|_{L^{1}(\Omega)}+2\left\|g \chi_{\left\{|g|>b_{k}\right\}}\right\|_{L^{1}(\Omega)},
\end{aligned}
$$

where $a_{k}=\frac{\alpha_{0}}{2} h(k)$ and $b_{k}=\frac{\alpha_{0}^{2}}{4} \sqrt{h(k)}$.

Set $l=\varphi_{\varepsilon}(j)$ : since $\varphi_{\varepsilon}$ is an increasing one-to-one bijection, and since $\varphi_{\varepsilon}^{-1}(s) \leq$ $c(s+\varepsilon)$, we deduce that

$$
\begin{aligned}
& \alpha \int_{\Omega} \mid D T_{l}\left(\left.\varphi_{\varepsilon}\left(G_{k}(u)\right)\right|^{p} d x\right. \\
& \leq(l+\varepsilon)\left(\gamma\left\|\left|D \varphi_{\varepsilon}\left(G_{k}(u)\right)\right|^{q}\right\|_{L^{1}(\Omega)}+\left\|f \chi_{\left\{|f|>a_{k}\right\}}\right\|_{L^{1}(\Omega)}+\|\mu\|_{\mathcal{M}_{b}(\Omega)}\right) \\
& \quad+\frac{2 \alpha_{0}}{\varepsilon \sqrt{h(k)}}\|g\|_{L^{1}(\Omega)}+2\left\|g \chi_{\left\{|g|>b_{k}\right\}}\right\|_{L^{1}(\Omega)}, \quad \forall l>0 .
\end{aligned}
$$

We apply now the well-known regularity lemma in Marcinkiewicz spaces due to [6] 
(see also the precised form in [8]), which gives

$$
\begin{aligned}
& \left\|\left|D \varphi_{\varepsilon}\left(G_{k}(u)\right)\right|^{p-1}\right\|_{M^{\frac{N}{N-1}}(\Omega)} \\
& \leq C \alpha^{-1}\left(\gamma\left\|\left|D \varphi_{\varepsilon}\left(G_{k}(u)\right)\right|^{q}\right\|_{L^{1}(\Omega)}+\left\|f \chi_{\left\{|f|>a_{k}\right\}}\right\|_{L^{1}(\Omega)}+\|\mu\|_{\mathcal{M}_{b}(\Omega)}\right) \\
& \quad+\alpha^{-\frac{1}{p^{\prime}}} \varepsilon^{\frac{1}{p^{\prime}}}\left(\gamma\left\|\left|D \varphi_{\varepsilon}\left(G_{k}(u)\right)\right|^{q}\right\|_{L^{1}(\Omega)}+\left\|f \chi_{\left\{|f|>a_{k}\right\}}\right\|_{L^{1}(\Omega)}+\|\mu\|_{\mathcal{M}_{b}(\Omega)}\right)^{\frac{1}{p^{\prime}}} \\
& \quad+\alpha^{-\frac{1}{p^{\prime}}}\left[\frac{2 \alpha_{0}}{\varepsilon \sqrt{h(k)}}\|g\|_{L^{1}(\Omega)}+2\left\|g \chi_{\left\{|g|>b_{k}\right\}}\right\|_{L^{1}(\Omega)}\right]^{\frac{1}{p^{\prime}}} .
\end{aligned}
$$

Since $q<\frac{N(p-1)}{N-1}$ we have that $L^{\frac{q}{p-1}}(\Omega) \subset M^{\frac{N}{N-1}}(\Omega)$, therefore we conclude, using also Young's inequality in the right-hand side,

$$
\begin{aligned}
& \left\|\left|D \varphi_{\varepsilon}\left(G_{k}(u)\right)\right|^{q}\right\|_{L^{1}(\Omega)}^{\frac{p-1}{q}} \\
& \leq C \alpha^{-1}\left(\gamma\left\|\left|D \varphi_{\varepsilon}\left(G_{k}(u)\right)\right|^{q}\right\|_{L^{1}(\Omega)}+\left\|f \chi_{\left\{|f|>a_{k}\right\}}\right\|_{L^{1}(\Omega)}+\|\mu\|_{\mathcal{M}_{b}(\Omega)}\right) \\
& \quad+C \varepsilon^{p-1}+\alpha^{-\frac{1}{p^{\prime}}}\left[\frac{2 \alpha_{0}}{\varepsilon \sqrt{h(k)}}\|g\|_{L^{1}(\Omega)}+2\left\|g \chi_{\left\{|g|>b_{k}\right\}}\right\|_{L^{1}(\Omega)}\right]^{\frac{1}{p^{\prime}}} .
\end{aligned}
$$

The last inequality still implies, for some constants $C_{1}, C_{2}$ :

$$
\begin{aligned}
C_{1} \alpha Y_{k}^{p-1}-C_{2} \gamma Y_{k}^{q} \leq & \left(\left\|f \chi_{\left\{|f|>a_{k}\right\}}\right\|_{L^{1}(\Omega)}+\|\mu\|_{\mathcal{M}_{b}(\Omega)}\right)+\alpha \varepsilon^{p-1} \\
& +\alpha^{\frac{1}{p}}\left[\frac{\alpha_{0}}{\varepsilon \sqrt{h(k)}}\|g\|_{L^{1}(\Omega)}+\left\|g \chi_{\left\{|g|>b_{k}\right\}}\right\|_{L^{1}(\Omega)}\right]^{\frac{1}{p^{\prime}}},
\end{aligned}
$$

where

$$
Y_{k}=\left\|\left|D \varphi_{\varepsilon}\left(G_{k}(u)\right)\right|^{q}\right\|_{L^{1}(\Omega)}^{\frac{1}{q}} .
$$

The conclusion follows as in the previous sections: we define the function

$$
F(Y)=C_{1} \alpha Y^{p-1}-C_{2} \gamma Y^{q},
$$

and we note that, since $q>p-1$, the function $F$ is concave with a maximum

$$
F^{*}=\max F(Y)=C^{*} \alpha^{\frac{q}{q-(p-1)}} \gamma^{\frac{p-1}{q-(p-1)}},
$$

for a constant $C^{*}$ which only depends on $p, q, N$. Then we distinguish between the two cases.

(i) If $\alpha_{0}=0$ we have $a_{k}=b_{k}=0$. Taking $\varepsilon=0$ the inequality (5.11) has the simpler form

$$
C_{1} \alpha Y_{k}^{p-1}-C_{2} \gamma Y_{k}^{q} \leq\|f\|_{L^{1}(\Omega)}+\|\mu\|_{\mathcal{M}_{b}(\Omega)}+\alpha^{\frac{1}{p}}\|g\|_{L^{1}(\Omega)}^{\frac{1}{p^{\prime}}},
$$


where now $Y_{k}=\left\|\left|D G_{k}(u)\right|^{q}\right\|_{L^{1}(\Omega)}^{\frac{1}{q}}$ since $\varphi_{0}(s)=s$. Here we use condition (5.4) which is nothing but

$$
\|f\|_{L^{1}(\Omega)}+\|\mu\|_{\mathcal{M}_{b}(\Omega)}+\alpha^{\frac{1}{p}}\|g\|_{L^{1}(\Omega)}^{\frac{1}{p^{\prime}}}<F^{*}=\max F(Y) .
$$

Then, with the usual continuity argument, we deduce an estimate for $Y_{0}$, i.e. for $\left\||D u|^{q}\right\|_{L^{1}(\Omega)}$, depending only on $\|f\|_{L^{1}(\Omega)},\|g\|_{L^{1}(\Omega)},\|\mu\|_{\mathcal{M}_{b}(\Omega)}$.

(ii) If $\alpha_{0}>0$ we use the Remark 5.1, i.e. we replace $\mu$ with its singular part $\mu_{s}$ up to changing $f$ and $g$. Then inequality (5.11) takes the form

$$
\begin{aligned}
& C_{1} \alpha Y_{k}^{p-1}-C_{2} \gamma Y_{k}^{q} \leq\left\|(|f|+|h|) \chi_{\left\{|f|+|h|>a_{k}\right\}}\right\|_{L^{1}(\Omega)}+\left\|\mu_{s}\right\|_{\mathcal{M}_{b}(\Omega)}+\alpha^{\frac{1}{p}} \varepsilon^{p-1} \\
& +\alpha^{\frac{1}{p}}\left[\frac{\alpha_{0}}{\varepsilon \sqrt{h(k)}}|| g+|H|^{p^{\prime}}\left\|_{L^{1}(\Omega)}+\right\|\left(g+|H|^{p^{\prime}}\right) \chi_{\left\{\left.|g+| H\right|^{p^{\prime}} \mid>b_{k}\right\}} \|_{L^{1}(\Omega)}\right]^{\frac{1}{p^{\prime}}}
\end{aligned}
$$

where we wrote the regular part of $\mu$ as $\mu_{0}=h+\operatorname{div}(H) \in L^{1}(\Omega)+W^{-1, p^{\prime}}(\Omega)$. Here we use (5.5), which is nothing but

$$
\left\|\mu_{s}\right\|_{\mathcal{M}_{b}(\Omega)}<F^{*} .
$$

Then we fix, successively, a small $\varepsilon$ and a large $k^{*}$ so that the right-hand side in (5.13) is still smaller than $F^{*}$. The continuity argument implies an estimate on $\left\|\left|D G_{k}(u)\right|^{q}\right\|_{L^{1}(\Omega)}$ for any $k>k^{*}$. An estimate on $T_{k}(u)$ in $W_{0}^{1, p}(\Omega)$ is then obtained as usual, and finally, once the term $H(x, u, D u)$ is estimated in $L^{1}(\Omega)$, all the standard estimates for equations with measure data follow.

In case $\alpha_{0}>0$, the previous result is not optimal, since we expect the size condition (5.5) required on $\left\|\mu_{s}\right\|_{\mathcal{M}_{b}(\Omega)}$ to be not necessary.

In this spirit, we give the following improved result in case $a(x, s, \xi)$ does not depend on $s$ and replacing assumptions (2.2)-(2.4) with the stronger conditions:

$$
\begin{aligned}
&\left(a(x, \xi)-a\left(x, \xi^{\prime}\right)\right)\left(\xi-\xi^{\prime}\right) \geq \alpha\left(|\xi|^{2}+\left|\xi^{\prime}\right|^{2}\right)^{\frac{p-2}{2}}\left|\xi-\xi^{\prime}\right|^{2}, \quad \alpha>0 \\
&\left\{\begin{array}{l}
|a(x, \xi)| \leq \beta\left[|\xi|^{p-1}+g(x)^{\frac{1}{p^{\prime}}}\right] \\
\beta>0, g(x) \in L^{1}(\Omega),
\end{array}\right.
\end{aligned}
$$

for almost every $x \in \Omega$ and for every $\xi, \xi^{\prime} \in \mathbb{R}^{N}$.

Note that (5.15) is the same as (2.3) when $a(x, s, \xi)$ does not depend on $s$; the function $\eta$ has now been called $g(x)^{\frac{1}{p^{\prime}}}$ because in this way (5.14)-(5.15) imply

$$
a(x, \xi) \cdot \xi \geq \alpha|\xi|^{p}+a(x, 0) \cdot \xi \geq \frac{\alpha}{2}|\xi|^{p}-C g(x)
$$

hence we recover (2.2) with a consistent notation. 
Actually, (5.14)-(5.15) are not the most general assumptions under which the following result can be proved, but they allow us for a few simplifications in some technical details.

Theorem 5.5. Assume (5.14), (5.15) with $p \geq 2$, and (2.5), (2.6), with $m=1$ and $p-1<q<\frac{N(p-1)}{N-1}$, and with $\alpha_{0}>0$. Let $u$ be a renormalized solution of

$$
\left\{\begin{array}{l}
-\operatorname{div}(a(x, D u))+a_{0}(x, u)=H(x, u, D u)+\mu \text { in } \Omega \\
u=0 \text { on } \partial \Omega
\end{array}\right.
$$

where $\mu \in \mathcal{M}_{b}(\Omega)$ and belongs to $L^{1}(\Omega)+W^{-1, p^{\prime}}(\Omega)$. Then we have

$$
\left\||u|^{p-1}\right\|_{M^{\frac{N}{N-p}}(\Omega)}+\left\||D u|^{p-1}\right\|_{M^{\frac{N}{N-1}}(\Omega)} \leq M
$$

where $M$ depends on $p, q, N, \alpha_{0},|\Omega|, \alpha, \gamma, \beta$ and $f, g,\|\mu\|_{\mathcal{M}_{b}(\Omega)}$. The constant $M$ remains bounded when $f$ and $g$ vary in sets $\mathcal{F}, \mathcal{G}$ which are bounded and equiintegrable in $L^{1}(\Omega)$.

Remark 5.6. In the above statement, the measure $\mu$ is assumed to belong to $L^{1}(\Omega)+W^{-1, p^{\prime}}(\Omega)$; however, the estimate only depends on $\|\mu\|_{\mathcal{M}_{b}(\Omega)}$. Therefore, the previous result can be used as an a priori estimate in case of approximating data converging to singular measures.

Proof.

Step 1: using the auxiliary function $v$.

Consider the (unique) renormalized solution $v$ of

$$
\begin{cases}-\operatorname{div}(a(x, D v)-a(x, 0))=\mu^{+} & \text {in } \Omega \\ v=0 & \text { on } \partial \Omega\end{cases}
$$

Since (5.14) implies $(a(x, \xi)-a(x, 0)) \cdot \xi \geq \alpha|\xi|^{p}$, one can easily prove that $v \geq 0$. Let now $\psi(t)$ be a nondecreasing Lipschitz function such that $\psi(0)=0$ and $\psi^{\prime}$ has compact support. Then we have

$$
\begin{aligned}
& \int_{\Omega}(a(x, D u)-a(x, D v)) D\left[\psi\left(G_{k}(u-v)^{+}\right)\right] d x \\
& \quad+\int_{\Omega} a_{0}(x, u) \psi\left(G_{k}(u-v)^{+}\right) d x \\
& =\int_{\Omega} H(x, u, D u) \psi\left(G_{k}(u-v)^{+}\right) d x \\
& \quad-\int_{\Omega} a(x, 0) D\left[\psi\left(G_{k}(u-v)^{+}\right)\right] d x
\end{aligned}
$$


Actually, it is not obvious that $\psi\left(G_{k}(u-v)^{+}\right)$can be taken as test function in (both) the equations of $u$ and $v$. However, since $\psi$ is bounded and since $\mu \in$ $L^{1}(\Omega)+W^{-1, p^{\prime}}(\Omega)$, this can be justified by some truncation arguments.

Observe that (since $\psi(0)=0$ ) all the integrals in (5.19) are restricted where $u>v+k$, in particular where $u$ is positive. This implies, thanks to (2.5),

$$
\int_{\Omega} a_{0}(x, u) \psi\left(G_{k}(u-v)^{+}\right) d x \geq \alpha_{0} \int_{\Omega} h(|u|) \psi\left(G_{k}(u-v)^{+}\right) d x .
$$

For the same reason we can use (2.6) to get

$$
\begin{aligned}
\int_{\Omega} H(x, u, D u) \psi\left(G_{k}(u-v)^{+}\right) d x \leq & \int_{\Omega}\left[\gamma|D u|^{q}+f(x)\right] \psi\left(G_{k}(u-v)^{+}\right) d x \\
\leq & C \gamma \int_{\Omega}\left|D G_{k}(u-v)^{+}\right|^{q} \psi\left(G_{k}(u-v)^{+}\right) d x \\
& +C \gamma \int_{\Omega}|D v|^{q} \psi\left(G_{k}(u-v)^{+}\right) d x \\
& +\int_{\Omega} f \psi\left(G_{k}(u-v)^{+}\right) d x .
\end{aligned}
$$

Moreover, (5.15) and Young's inequality imply

$$
\begin{aligned}
\left|\int_{\Omega} a(x, 0) D\left[\psi\left(G_{k}(u-v)^{+}\right)\right] d x\right| \leq & \left.\beta \int_{\Omega}|g|^{\frac{1}{p^{\prime}}} \mid D G_{k}(u-v)^{+}\right) \mid \psi^{\prime}\left(G_{k}(u-v)^{+}\right) d x \\
\leq & C \int_{\Omega}|g| \psi^{\prime}\left(G_{k}(u-v)^{+}\right) d x \\
& \left.+\delta \int_{\Omega} \mid D G_{k}(u-v)^{+}\right)\left.\right|^{p} \psi^{\prime}\left(G_{k}(u-v)^{+}\right) d x,
\end{aligned}
$$

where $\delta$ is a small number to be fixed later.

Therefore, if we set $w:=(u-v)^{+}$, we obtain from (5.19), using also (5.14) in the first term,

$$
\begin{aligned}
& \alpha \int_{\Omega}\left(|D u|^{2}+|D v|^{2}\right)^{\frac{p-2}{2}}\left|D\left(G_{k}(w)\right)\right|^{2} \psi^{\prime}\left(G_{k}(w)\right) d x+\alpha_{0} \int_{\Omega} h(|u|) \psi\left(G_{k}(w)\right) d x \\
& \leq C \gamma \int_{\Omega}\left|D G_{k}(w)\right|^{q} \psi\left(G_{k}(w)\right) d x \\
& \quad+C \gamma \int_{\Omega}|D v|^{q} \psi\left(G_{k}(w)\right) d x+\int_{\Omega} f \psi\left(G_{k}(w)\right) d x \\
& \quad+C \int_{\Omega}|g| \psi^{\prime}\left(G_{k}(w)\right) d x+\delta \int_{\Omega}\left|D G_{k}(w)\right|^{p} \psi^{\prime}\left(G_{k}(w)\right) d x .
\end{aligned}
$$


We choose here as in the previous theorem

$$
\psi(t)=T_{j}(t) \frac{\left|T_{j}(t)\right|}{\varepsilon+\left|T_{j}(t)\right|},
$$

and we obtain, after obvious simplifications,

$$
\begin{aligned}
& \alpha \int_{\Omega}\left(|D u|^{2}+|D v|^{2}\right)^{\frac{p-2}{2}}\left|D T_{j}\left(G_{k}(w)\right)\right|^{2} \frac{T_{j}\left(G_{k}(w)\right)}{\varepsilon+T_{j}\left(G_{k}(w)\right)} d x \\
& \quad+\alpha_{0} \int_{\Omega} h(|u|) \frac{T_{j}\left(G_{k}(w)\right)^{2}}{\varepsilon+T_{j}\left(G_{k}(w)\right)} d x \\
& \leq C \gamma \int_{\Omega}\left|D G_{k}(w)\right|^{q} \frac{T_{j}\left(G_{k}(w)\right)^{2}}{\varepsilon+T_{j}\left(G_{k}(w)\right)} d x \\
& \quad+C \gamma \int_{\Omega}|D v|^{q} \frac{T_{j}\left(G_{k}(w)\right)^{2}}{\varepsilon+T_{j}\left(G_{k}(w)\right)} d x+\int_{\Omega}|f| \frac{T_{j}\left(G_{k}(w)\right)^{2}}{\varepsilon+T_{j}\left(G_{k}(w)\right)} d x \\
& \quad+2 C \int_{\Omega}|g| \frac{T_{j}\left(G_{k}(w)\right)}{\varepsilon+T_{j}\left(G_{k}(w)\right)} d x+2 \delta \int_{\Omega}\left|D T_{j}\left(G_{k}(w)\right)\right|^{p} \frac{T_{j}\left(G_{k}(w)\right)}{\varepsilon+T_{j}\left(G_{k}(w)\right)} d x .
\end{aligned}
$$

Since $v \geq 0$, we have $\{w>k\} \subset\{u>k\}$, hence we split the term with $f$ as usual

$$
\begin{aligned}
\int_{\Omega}|f| \frac{T_{j}\left(G_{k}(w)\right)^{2}}{\varepsilon+T_{j}\left(G_{k}(w)\right)} d x \leq & \frac{\alpha_{0}}{4} \int_{\Omega} h(u) \frac{T_{j}\left(G_{k}(w)\right)^{2}}{\varepsilon+T_{j}\left(G_{k}(w)\right)} d x \\
& +\int_{\left\{|f|>\frac{\alpha_{0}}{4} h(u)\right\}}|f| \frac{T_{j}\left(G_{k}(w)\right)^{2}}{\varepsilon+T_{j}\left(G_{k}(w)\right)} d x \\
& \leq \frac{\alpha_{0}}{4} \int_{\Omega} h(|u|) \frac{T_{j}\left(G_{k}(w)\right)^{2}}{\varepsilon+T_{j}\left(G_{k}(w)\right)} d x+j \int_{E_{k}}|f| d x,
\end{aligned}
$$

where $E_{k}=\left\{|f|>\frac{\alpha_{0}}{4} h(k)\right\}$. Similarly we deal with the term $|D v|^{q}$, obtaining

$$
\begin{aligned}
C \gamma \int_{\Omega}|D v|^{q} \frac{T_{j}\left(G_{k}(w)\right)^{2}}{\varepsilon+T_{j}\left(G_{k}(w)\right)} d x \leq & \frac{\alpha_{0}}{4} \int_{\Omega} h(|u|) \frac{T_{j}\left(G_{k}(w)\right)^{2}}{\varepsilon+T_{j}\left(G_{k}(w)\right)} d x \\
& +C \gamma j \int_{F_{k}}|D v|^{q} d x,
\end{aligned}
$$

where $F_{k}=\left\{C \gamma|D v|^{q}>\frac{\alpha_{0}}{4} h(k)\right\}$. 
The term with $g$ can also be dealt with as in the previous theorem, hence we get

$$
\begin{aligned}
C \int_{\Omega}|g| \frac{T_{j}\left(G_{k}(w)\right)}{\varepsilon+T_{j}\left(G_{k}(w)\right)} d x \leq & \frac{\alpha_{0}}{2} \int_{\Omega} h(|u|) \frac{T_{j}\left(G_{k}(w)\right)^{2}}{\varepsilon+T_{j}\left(G_{k}(w)\right)} d x \\
& +C \frac{\alpha_{0}}{\varepsilon \sqrt{h(k)}}\|g\|_{L^{1}(\Omega)}+C \int_{G_{k}}|g| d x,
\end{aligned}
$$

where $G_{k}=\left\{|g|>\frac{\alpha_{0}^{2}}{4} \sqrt{h(k)}\right\}$. We then deduce the inequality

$$
\begin{aligned}
& \alpha \int_{\Omega}\left(|D u|^{2}+|D v|^{2}\right)^{\frac{p-2}{2}}\left|D T_{j}\left(G_{k}(w)\right)\right|^{2} \frac{T_{j}\left(G_{k}(w)\right)}{\varepsilon+T_{j}\left(G_{k}(w)\right)} d x \\
& \leq C \gamma \int_{\Omega}\left|D G_{k}(w)\right|^{q} \frac{T_{j}\left(G_{k}(w)\right)^{2}}{\varepsilon+T_{j}\left(G_{k}(w)\right)} d x \\
& \quad+C j\left(\int_{E_{k}}|f| d x+\int_{F_{k}}|D v|^{q} d x\right) \\
& \quad+C \frac{\alpha_{0}}{\varepsilon \sqrt{h(k)}}\|g\|_{L^{1}(\Omega)}+C \int_{G_{k}}|g| d x \\
& \quad+2 \delta \int_{\Omega}\left|D T_{j}\left(G_{k}(w)\right)\right|^{p} \frac{T_{j}\left(G_{k}(w)\right)}{\varepsilon+T_{j}\left(G_{k}(w)\right)} d x .
\end{aligned}
$$

Step 2: Marcinkiewicz estimate for $\left|D G_{k}(u-v)^{+}\right|$.

Since $p \geq 2$, we have $\left(|\xi|^{2}+|\eta|^{2}\right)^{\frac{p-2}{2}}|\xi-\eta|^{2} \geq\left(\frac{1}{2}\right)^{\frac{p-2}{2}}|\xi-\eta|^{p}$ hence we obtain from (5.20), choosing a suitable small $\delta$,

$$
\begin{aligned}
\int_{\Omega}\left|D T_{j}\left(G_{k}(w)\right)\right|^{p} \frac{T_{j}\left(G_{k}(w)\right)}{\varepsilon+T_{j}\left(G_{k}(w)\right)} d x \leq & C \int_{\Omega}\left|D G_{k}(w)\right|^{q} \frac{T_{j}\left(G_{k}(w)\right)^{2}}{\varepsilon+T_{j}\left(G_{k}(w)\right)} d x \\
& +C j\left(\int_{E_{k}}|f| d x+\int_{F_{k}}|D v|^{q} d x\right) \\
& +C \frac{\alpha_{0}}{\varepsilon \sqrt{h(k)}}\|g\|_{L^{1}(\Omega)}+C \int_{G_{k}}|g| d x .
\end{aligned}
$$

We proceed henceforth as in the previous proof to obtain an estimate on $\left|D G_{k}(w)\right|$ in $L^{q}(\Omega)$ for $k \geq k^{*}$, where $k^{*}$ depends on (the equi-integrability of) $f, g$ and 
$|D v|^{q}$. On the other hand, the norm of $|D v|^{p-1}$ in the Marcinkiewicz space $M^{\frac{N}{N-1}}$ only depends on $\|\mu\|_{\mathcal{M}_{b}(\Omega)}$, and so is the equi-integrability of $|D v|^{q}$, since $q<$ $\frac{N(p-1)}{N-1}$. We deduce from that an estimate on $\left|D G_{k}(w)\right|=\left|D G_{k}(u-v)^{+}\right|$in $L^{q}(\Omega)$ for $k \geq k^{*}$, where $k^{*}$ depends on $\|\mu\|_{\mathcal{M}_{b}(\Omega)}$, and on the equi-integrability of $f$ and $g$.

Step 3: estimate when $0<u<v$.

Now we multiply the equation of $u$ by $1-\left(1+u^{+}\right)^{-\lambda}$ obtaining

$$
\begin{aligned}
& \lambda \int_{\Omega} a(x, D u) D u^{+}\left(1+u^{+}\right)^{-\lambda-1} d x+\int_{\Omega} a_{0}(x, u)\left[1-\left(1+u^{+}\right)^{-\lambda}\right] d x \\
& =\int_{\Omega} H(x, u, D u)\left[1-\left(1+u^{+}\right)^{-\lambda}\right] d x+\|\mu\|_{\mathcal{M}_{b}(\Omega)} .
\end{aligned}
$$

Observe that this test function only charges where $u \geq 0$, hence we get, using (5.14)-(5.15),

$$
\begin{aligned}
\alpha \lambda \int_{\Omega}\left|D u^{+}\right|^{p}\left(1+u^{+}\right)^{-\lambda-1} d x \leq & \gamma \int_{\Omega}\left|D u^{+}\right|^{q} d x \\
& +\left(\|f\|_{L^{1}(\Omega)}+\|\mu\|_{\mathcal{M}_{b}(\Omega)}\right) \\
& +\beta \int_{\Omega}|g|^{\frac{1}{p^{\prime}}}\left|D u^{+}\right|\left(1+u^{+}\right)^{-\lambda-1}
\end{aligned}
$$

which yields

$$
\begin{aligned}
& \frac{\alpha \lambda}{2} \int_{\Omega}\left|D u^{+}\right|^{p}\left(1+u^{+}\right)^{-\lambda-1} d x \\
& \leq C \int_{\{u \leq v+k\}}\left|D u^{+}\right|^{q} d x+C \int_{\Omega}\left|D G_{k}(u-v)^{+}\right|^{q} d x \\
& \quad+C \int_{\Omega}|D v|^{q} d x+\left(\|f\|_{L^{1}(\Omega)}+\|\mu\|_{\mathcal{M}_{b}(\Omega)}\right)+C \int_{\Omega}|g| d x .
\end{aligned}
$$

Since

$$
\begin{aligned}
C \int_{\{u \leq v+k\}}\left|D u^{+}\right|^{q} d x \leq & \frac{\alpha \lambda}{4} \int_{\Omega}\left|D u^{+}\right|^{p}\left(1+u^{+}\right)^{-\lambda-1} d x \\
& +C \int_{\{0 \leq u \leq v+k\}}\left(1+u^{+}\right)^{(\lambda+1) \frac{q}{p-q}} d x
\end{aligned}
$$


and since in the last integral we can use that $u \leq v+k$ we obtain

$$
\begin{aligned}
& \frac{\alpha \lambda}{4} \int_{\Omega}\left|D u^{+}\right|^{p}\left(1+u^{+}\right)^{-\lambda-1} d x \\
& \leq C \int_{\Omega}(1+v+k)^{(\lambda+1) \frac{q}{p-q}} d x+C \int_{\Omega}\left|D G_{k}(u-v)^{+}\right|^{q} d x \\
& \quad+C \int_{\Omega}|D v|^{q} d x+\left(\|f\|_{L^{1}(\Omega)}+\|\mu\|_{\mathcal{M}_{b}(\Omega)}\right)+C \int_{\Omega}|g| d x .
\end{aligned}
$$

Since $q<\frac{N(p-1)}{N-1}$ we have $(\lambda+1) \frac{q}{p-q}<\frac{N(p-1)}{N-p}$ for any positive $\lambda$ close to zero. Therefore the first integral in the right-hand side is bounded because of the estimates on $v$. Taking $k=k^{*}$ the second integral is also bounded by the previous step, and so all the right-hand side is bounded by constants only depending on $\|\mu\|_{\mathcal{M}_{b}(\Omega)},\|f\|_{L^{1}(\Omega)},\|g\|_{L^{1}(\Omega)}$ and on the equi-integrability of $f$ and $g$. We conclude an estimate for

$$
\int_{\Omega}\left|D u^{+}\right|^{p}\left(1+u^{+}\right)^{-\lambda-1} d x
$$

for any positive $\lambda$ close to zero; which implies (this is the classical argument in [12]) an estimate of $\left|D u^{+}\right|^{p-1}$ in $L^{r}(\Omega)$ for any $r<\frac{N}{N-1}$. In particular we conclude that $\left|D u^{+}\right|^{q}$ is estimated in $L^{1}(\Omega)$.

Step 4: conclusion.

Reasoning on $u^{-}$in the same way we obtain an estimate for $\left|D u^{-}\right|^{q}$, hence we conclude that $|D u|^{q}$ satisfies an estimate in $L^{1}(\Omega)$. Thanks to (2.6), we conclude by the usual theory with measure data all the desired estimates.

In consequence of the previous estimates, we can prove the existence of solutions to problem (5.1). To this purpose, we need to take care of the growth of $a_{0}(x, u)$ in case $\mu$ contains a singular part $\mu_{s} \neq 0$, by requiring, for some constant $C>0$ :

$$
\left|a_{0}(x, u)\right| \leq C\left(\tilde{a}_{0}(x)+|u|^{s}\right) \quad \text { with } s<\frac{N(p-1)}{N-p}, \tilde{a}_{0}(x) \in L^{1}(\Omega) .
$$

Corollary 5.7. Assume (2.2)-(2.6), with $r=1, m=1$ and $q<\frac{N(p-1)}{N-1}$, and (3.34), (3.35). Moreover assume that one of the following assumptions is satisfied:

(i) $\alpha_{0}=0$ and (5.4) holds true.

(ii) $\alpha_{0}>0$ and (5.5) holds true.

(ii) $\alpha_{0}>0$ and (5.14)-(5.15) hold true, with $p \geq 2$.

Furthermore, in case that $\mu_{s} \neq 0$, let (5.21) hold true. Then there exists a renormalized solution $u$ of (5.1). 
Proof. Take a sequence of approximating solutions $u_{n} \in W_{0}^{1, p}(\Omega)$ of the problem

$$
\left\{\begin{array}{l}
-\operatorname{div}\left(a\left(x, u_{n}, D u_{n}\right)\right)+a_{0}\left(x, u_{n}\right)=H\left(x, u_{n}, D u_{n}\right)+\mu_{n} \text { in } \Omega, \\
u_{n}=0 \text { on } \partial \Omega,
\end{array}\right.
$$

where $\mu_{n}$ is a suitable approximation of $\mu$ constructed by convolution, as in [22].

Applying Theorem 5.3 or Theorem 5.5, we deduce the estimates on $u_{n}$ and $\left|D u_{n}\right|$ in Marcinkiewicz spaces, hence (3.35) implies that $T_{n}\left(H\left(x, u_{n}, D u_{n}\right)\right)$ is bounded in $L^{1}(\Omega)$. Using the compactness and stability results of the theory of elliptic equations with measure data (see $[12,22]$ and references therein) we obtain that, up to extracting a subsequence, $u_{n}$ converges a.e. to some $u \in \mathcal{T}_{0}^{1, p}(\Omega)$, and $D u_{n}$ converges to $D u$ almost everywhere in $\Omega$. As a consequence of (3.35), since $q<\frac{N(p-1)}{N-1}$, the term $T_{n}\left(H\left(x, u_{n}, D u_{n}\right)\right)$ strongly converges in $L^{1}(\Omega)$ to $H(x, u, D u)$. If $\mu_{s}=0$, since the right-hand side is compact in $L^{1}(\Omega)+W^{-1, p^{\prime}}(\Omega)$, we also deduce that $a_{0}\left(x, u_{n}\right)$ converges in $L^{1}(\Omega)$ to $a_{0}(x, u)$ using (3.34); if $\mu_{s} \neq$ 0 , we use the growth condition (5.21) to deduce such a convergence. Still using the results in [22], we have that the truncations $T_{k}\left(u_{n}\right)$ strongly converge to $T_{k}(u)$ in $W_{0}^{1, p}(\Omega)$ for any $k>0$ and we conclude that passing to the limit $u$ satisfies (5.2) and (5.3), i.e. it is a renormalized solution.

We conclude this section with the statement of a regularity result as in Theorem 4.9. Indeed, the same regularity as in this latter theorem can be proved to hold when $q<\frac{N(p-1)}{N-1}$; the only difference is that now we do not ask more regularity on the solutions than what is needed in the definition of renormalized solutions. Actually, when $q<\frac{N(p-1)}{N-1}$, the bootstrap argument works as if the equation does not contain the term $H(x, u, D u)$. We omit the details of the proof, which follows the steps of Theorem 4.9 .

Theorem 5.8. Assume (2.2), (2.3), (2.5), (2.6) with $q<\frac{N(p-1)}{N-1}$. Assume in addition that $f$ belongs to $L^{s}(\Omega)$, with $s>1$ and $g$ belongs to $L^{t}(\Omega)$, with $t \geq 1$. Let $u$ be a renormalized solution of (5.1). Then the conclusion of Theorem 4.9 holds true.

\section{The case $p \geq N$}

Let us consider here the case that $p \geq N$. First of all, we observe that the case $p=N$ can be dealt with as in the previous section. Indeed, when $p=N$ we have $\frac{N(p-1)}{N-1}=(p-1)+\frac{p}{N}=p$, hence assuming $q<p$ implies that $q<\frac{N(p-1)}{N-1}$; we obtain then the following

Theorem 6.1. Let $p=N$. Assume (2.2), (2.3), (2.5), (2.6), with $r=1, m=1$ and $p-1<q<p$. Assume further that one of the following assumptions is satisfied

(i) $\alpha_{0}=0$ and (5.4) holds true.

(ii) $\alpha_{0}>0$ and (5.5) holds true. 
(ii) $\alpha_{0}>0$ and (5.14)-(5.15) hold true.

Let $u$ be a renormalized solution of (5.1). Then, given any $s<\infty$ and $t<N$ we have

$$
\|u\|_{M^{s}(\Omega)}+\||D u|\|_{M^{t}(\Omega)} \leq M
$$

where $M$ depends on $s, t, p, q, \alpha_{0}, \alpha, \gamma, \beta,|\Omega|$ and $f, g, \mu$. When $\alpha_{0}=0$ the constant $M$ depends on $f, g, \mu$ only through $\|f\|_{L^{1}(\Omega)},\|g\|_{L^{1}(\Omega)}$ and $\|\mu\|_{\mathcal{M}_{b}(\Omega)}$. When $\alpha_{0}>0$, the constant $M$ does not depend only on $\|f\|_{L^{1}(\Omega)},\|g\|_{L^{1}(\Omega)}$, $\|\mu\|_{\mathcal{M}_{b}(\Omega)}$, but remains bounded when $f$ and $g$ vary in sets which are bounded and equi-integrable in $L^{1}(\Omega)$ and $\mu_{0}$ varies in a compact set of $L^{1}(\Omega)+W^{-1, p^{\prime}}(\Omega)$. Moreover, assume in addition (3.34)-(3.35) and, if $\mu_{s} \neq 0$, also that

$$
\left|a_{0}(x, u)\right| \leq C\left(\tilde{a}_{0}(x)+|u|^{s}\right) \quad \text { for some } s<\infty, \tilde{a}_{0}(x) \in L^{1}(\Omega) .
$$

Then there exists a renormalized solution $u$ of (5.1).

We are left with the case $p>N$. Note that this implies that $\mathcal{M}_{b}(\Omega) \subset$ $W^{-1, p^{\prime}}(\Omega)$, hence data in Lebesgue spaces, or even measure data, always yield finite energy solutions, in the sense of (3.1). Moreover, in this situation solutions in $W_{0}^{1, p}(\Omega)$ are bounded as well, and the case $q<p$ does not really differ from the case $q=p$.

Theorem 6.2. Assume (2.2), (2.3), (2.5), (2.6) with $p-1<q \leq p, m=1$ and $r=1$. Assume further that either

(i) $\alpha_{0}>0$,

or

(ii) $\alpha_{0}=0$ and

$$
\alpha^{-\frac{1}{p-1}}\|f\|_{L^{1}(\Omega)}^{p^{\prime}}+\|g\|_{L^{1}(\Omega)}<C^{*} \alpha^{\frac{q+1}{q-(p-1)}} \gamma^{-\frac{p}{q-(p-1)}}
$$

where $C^{*}$ is a constant depending only on $p, q, N$ which will be defined in (6.6).

Let $u \in W_{0}^{1, p}(\Omega)$ be a solution of (2.1). Then

$$
\|u\|_{W_{0}^{1, p}(\Omega)} \leq M
$$

where $M$ depends on $p, q, N, \alpha_{0}, \alpha, \gamma, \Omega$ and the data $f, g$. When $\alpha_{0}=0$ the constant $M$ depends on $f$ and $g$ only through $\|f\|_{L^{1}(\Omega)}$ and $\|g\|_{L^{1}(\Omega)}$. When $\alpha_{0}>0$, the constant $M$ does not depend only on $\|f\|_{L^{1}(\Omega)}$ and $\|g\|_{L^{1}(\Omega)}$ but remains bounded when $f$ and $g$ vary in sets which are bounded and equi-integrable in $L^{1}(\Omega)$.

Proof. We proceed as in the proof of Theorem 3.1 for the case $q=p-1+\frac{p}{N}$. If $\alpha_{0}=0$ it would be enough to choose as test function $v=G_{k}(u)$ in (2.1). In order to treat the case $\alpha_{0}>0$, we choose the test function

$$
v=G_{k}(u) \frac{\left|G_{k}(u)\right|}{\varepsilon+\left|G_{k}(u)\right|} .
$$


We obtain, using (2.6), (2.2) and (2.5),

$$
\begin{aligned}
& \alpha \int_{\Omega}|D u|^{p} \frac{\left|G_{k}(u)\right|}{\varepsilon+\left|G_{k}(u)\right|} d x+\alpha_{0} \int_{\Omega} h(|u|) \frac{G_{k}(u)^{2}}{\varepsilon+\left|G_{k}(u)\right|} d x \\
& \leq \gamma \int_{\Omega}|D u|^{q} \frac{G_{k}(u)^{2}}{\varepsilon+\left|G_{k}(u)\right|} d x+\int_{\Omega}|f| \frac{G_{k}(u)^{2}}{\varepsilon+\left|G_{k}(u)\right|} d x \\
& \quad+2 \int_{\Omega}|g| \frac{G_{k}(u)}{\varepsilon+\left|G_{k}(u)\right|} d x .
\end{aligned}
$$

Then, splitting the terms with $f$ and $g$ as usual we get

$$
\begin{aligned}
\alpha \int_{\Omega}|D u|^{p} \frac{\left|G_{k}(u)\right|}{\varepsilon+\left|G_{k}(u)\right|} d x \leq & \gamma \int_{\Omega}|D u|^{q} \frac{G_{k}(u)^{2}}{\varepsilon+\left|G_{k}(u)\right|} d x \\
& +\int_{\left\{|f|>\frac{\alpha_{0}}{2} h(k)\right\}}|f| \frac{G_{k}(u)^{2}}{\varepsilon+\left|G_{k}(u)\right|} d x \\
& +\frac{2 \alpha_{0}}{\varepsilon \sqrt{h(k)}}\|g\|_{L^{1}(\Omega)}+2 \int_{\left\{|g|>\frac{\alpha_{0}^{2}}{4} \sqrt{h(k)}\right\}}|g| d x .
\end{aligned}
$$

We introduce the function

$$
\varphi_{\varepsilon}(s)=\int_{0}^{s}\left(\frac{|\xi|}{\varepsilon+|\xi|}\right)^{\frac{1}{p}} d \xi
$$

hence we obtain

$$
\begin{aligned}
\alpha \int_{\Omega} \mid D\left(\left.\varphi_{\varepsilon}\left(G_{k}(u)\right)\right|^{p} d x \leq\right. & \gamma \int_{\Omega}\left|D \varphi_{\varepsilon}\left(G_{k}(u)\right)\right|^{q} G_{k}(u)\left(\frac{\left|G_{k}(u)\right|}{\varepsilon+\left|G_{k}(u)\right|}\right)^{1-\frac{q}{p}} d x \\
& +\int_{\left\{|f|>\frac{\alpha_{0}}{2} h(k)\right\}}|f| \frac{G_{k}(u)^{2}}{\varepsilon+\left|G_{k}(u)\right|} d x+\frac{2 \alpha_{0}}{\varepsilon \sqrt{h(k)}}\|g\|_{L^{1}(\Omega)} \\
& +2 \int_{\left\{|g|>\frac{\alpha_{0}^{2}}{4} \sqrt{h(k)}\right\}}|g| d x .
\end{aligned}
$$

Recall that

$$
s \leq c\left(\varphi_{\varepsilon}(s)+\varepsilon\right), \quad \frac{s^{2}}{\varepsilon+s} \leq c \varphi_{\varepsilon}(s),
$$


so that we get

$$
\begin{aligned}
\alpha \int_{\Omega} \mid D\left(\left.\varphi_{\varepsilon}\left(G_{k}(u)\right)\right|^{p} d x \leq\right. & \gamma C \int_{\Omega}\left|D \varphi_{\varepsilon}\left(G_{k}(u)\right)\right|^{q}\left(\left|\varphi_{\varepsilon}\left(G_{k}(u)\right)\right|+\varepsilon\right) d x \\
& +C \int_{\left\{|f|>\frac{\alpha_{0}}{2} h(k)\right\}}|f|\left|\varphi_{\varepsilon}\left(G_{k}(u)\right)\right| d x \\
& +\frac{2 \alpha_{0}}{\varepsilon \sqrt{h(k)}}\|g\|_{L^{1}(\Omega)}+2 \int_{\left\{|g|>\frac{\alpha_{0}^{2}}{4} \sqrt{h(k)}\right\}}|g| d x \\
\leq & C \gamma\left\|D \varphi_{\varepsilon}\left(G_{k}(u)\right)\right\|_{L^{p}(\Omega)}^{q}\left(\left\|\varphi_{\varepsilon}\left(G_{k}(u)\right)\right\|_{L^{\infty}(\Omega)}+\varepsilon\right) \\
& +C\left\|f \chi_{\left\{|f|>\frac{\alpha_{0}}{2} h(k)\right\}}\right\|_{L^{1}(\Omega)}\left\|\varphi_{\varepsilon}\left(G_{k}(u)\right)\right\|_{L^{\infty}(\Omega)} \\
& +\frac{2 \alpha_{0}}{\varepsilon \sqrt{h(k)}}+2\left\|g \chi_{\left\{|g|>\frac{\alpha_{0}^{2}}{4} \sqrt{h(k)\}}\right.}\right\|_{L^{1}(\Omega)} .
\end{aligned}
$$

Recalling that $W_{0}^{1, p}(\Omega) \subset L^{\infty}(\Omega)$, we end up with

$$
\begin{aligned}
\alpha\left\|\varphi_{\varepsilon}\left(G_{k}(u)\right)\right\|_{W_{0}^{1, p}(\Omega)}^{p} \leq & C \gamma\left\|\varphi_{\varepsilon}\left(G_{k}(u)\right)\right\|_{W_{0}^{1, p}(\Omega)}^{q}\left(\left\|\varphi_{\varepsilon}\left(G_{k}(u)\right)\right\|_{W_{0}^{1, p}(\Omega)}+\varepsilon\right) \\
& +C\left\|f \chi_{\left\{|f|>a_{k}\right\}}\right\|_{L^{1}(\Omega)}\left\|\varphi_{\varepsilon}\left(G_{k}(u)\right)\right\|_{W_{0}^{1, p}(\Omega)} \\
& +\frac{2}{\varepsilon} \frac{\alpha_{0}}{\sqrt{h(k)}}\|g\|_{L^{1}(\Omega)}+2\left\||g| \chi_{\left\{|g|>b_{k}\right\}}\right\|_{L^{1}(\Omega)},
\end{aligned}
$$

where $a_{k}=\frac{\alpha_{0}}{2} h(k)$ and $b_{k}=\frac{\alpha_{0}^{2}}{4} \sqrt{h(k)}$. Using Young's inequality we obtain, for some constants $C_{0}, C_{1}$ :

$\alpha C_{0}\left\|\varphi_{\varepsilon}\left(G_{k}(u)\right)\right\|_{W_{0}^{1, p}(\Omega)}^{p}-\gamma C_{1}\left\|\varphi_{\varepsilon}\left(G_{k}(u)\right)\right\|_{W_{0}^{1, p}(\Omega)}^{q+1}$

$\leq \gamma \varepsilon^{q+1}+\alpha^{-\frac{1}{p-1}}\left\|f \chi_{\left\{|f|>a_{k}\right\}}\right\|_{L^{1}(\Omega)}^{p^{\prime}}+\frac{1}{\varepsilon} \frac{\alpha_{0}}{\sqrt{h(k)}}\|g\|_{L^{1}(\Omega)}+\left\||g| \chi_{\left\{|g|>b_{k}\right\}}\right\|_{L^{1}(\Omega)}$.

Set now

$$
Y_{k}=\left\|\varphi_{\varepsilon}\left(G_{k}(u)\right)\right\|_{W_{0}^{1, p}(\Omega)} .
$$

Then, the inequality takes the form

$$
\begin{aligned}
F\left(Y_{k}\right) \leq & \gamma \varepsilon^{q+1}+\alpha^{-\frac{1}{p-1}}\left\|f \chi_{\left\{|f|>a_{k}\right\}}\right\|_{L^{1}(\Omega)}^{p^{\prime}} \\
& +\frac{1}{\varepsilon} \frac{\alpha_{0}}{\sqrt{h(k)}}\|g\|_{L^{1}(\Omega)}+\left\||g| \chi_{\left\{|g|>b_{k}\right\}}\right\|_{L^{1}(\Omega)},
\end{aligned}
$$


where

$$
F(t)=\alpha C_{0} t^{p}-\gamma C_{1} t^{q+1} .
$$

Note that $p-1<q$, so that $F$ is a concave function with a unique maximum

$$
F^{*}=\max F(Y)=C^{*} \alpha^{\frac{q+1}{q-(p-1)}} \gamma^{-\frac{p}{q-(p-1)}}
$$

(i) If $\alpha_{0}=0$ we can take $\varepsilon=0$ and (since we also have $a_{k}=b_{k}=0$ ) we obtain the simpler inequality

$$
\alpha C_{0}\left\|G_{k}(u)\right\|_{W_{0}^{1, p}(\Omega)}^{p}-\gamma C_{1}\left\|G_{k}(u)\right\|_{W_{0}^{1, p}(\Omega)}^{q+1} \leq \alpha^{-\frac{1}{p-1}}\|f\|_{L^{1}(\Omega)}^{p^{\prime}}+\|g\|_{L^{1}(\Omega)} .
$$

We use (6.2) which is nothing but

$$
\alpha^{-\frac{1}{p-1}}\|f\|_{L^{1}(\Omega)}^{p^{\prime}}+\|g\|_{L^{1}(\Omega)}<F^{*}
$$

Then the usual continuity argument implies the estimate for $\left\|G_{k}(u)\right\|_{W_{0}^{1, p}(\Omega)}$ up to $k=0$, i.e. for $\|u\|_{W_{0}^{1, p}(\Omega)}$.

(ii) If $\alpha_{0}>0$ we fix $\varepsilon=\varepsilon_{0}$ as the minimum point of the right-hand side in (6.5), which gives

$$
F\left(Y_{k}\right) \leq M_{k}
$$

where

$$
M_{k}=C \gamma^{\frac{1}{q+2}}\left(\frac{\alpha_{0}\|g\|_{L^{1}(\Omega)}}{\sqrt{h(k)}}\right)^{\frac{q+1}{q+2}}+\alpha^{-\frac{1}{p-1}}\left\|f \chi_{\left\{|f|>a_{k}\right\}}\right\|_{L^{1}(\Omega)}^{p^{\prime}}+\left\|g \chi_{\left\{|g|>b_{k}\right\}}\right\|_{L^{1}(\Omega)} .
$$

Then we define $k^{*}$ so that $M_{k}<F^{*}$ for every $k>k^{*}$. Now either we have $\|u\|_{\infty} \leq k^{*}$, which means an estimate of $u$ in $L^{\infty}(\Omega)$; in that case we obtain soon from the equation an estimate on $u$ in $W_{0}^{1, p}(\Omega)$ and we conclude. Otherwise we have $k^{*}<\|u\|_{L^{\infty}(\Omega)}$ and the inequality

$$
F\left(Y_{k}\right)<M_{k} \quad \forall k \in\left(k^{*},\|u\|_{L^{\infty}(\Omega)}\right),
$$

implies, with the continuity argument, an estimate on $Y_{k^{*}}$, hence we estimate $\left\|G_{k^{*}+1}(u)\right\|_{W_{0}^{1, p}(\Omega)}$. We deduce then an estimate for $\|u\|_{L^{\infty}(\Omega)}$, and then from the equation we easily complete the estimate on $u$ in $W_{0}^{1, p}(\Omega)$.

Remark 6.3. Note that the above result includes the case of measure data $\mu$ as right-hand side in the equation. Since $p>N$, we have $\mu \in W^{-1, p^{\prime}}(\Omega)$ and this term can be included in the operator $\operatorname{div}(a(x, s, \xi))$. 


\section{Further remarks}

\subsection{Other boundary conditions}

The same method introduced above also applies to problems with different boundary conditions. Here, we assume that $\Omega$ is a smooth bounded set, and we consider the equation

$$
\left\{\begin{array}{l}
-\operatorname{div}(a(x, u, D u))+a_{0}(x, u)=H(x, u, D u) \text { in } \Omega, \\
a(x, u, D u) \cdot v+\mu u=\omega \text { on } \partial \Omega
\end{array}\right.
$$

where $v(x)$ is the outward unit normal at $\partial \Omega, \mu$ is a nonnegative constant, and $\omega \in W^{1-\frac{1}{m}, m}(\partial \Omega)$. This means that

$$
\exists w \in W^{1, m}(\Omega): \quad \omega=\gamma_{\partial \Omega}(w),
$$

where $\gamma_{\partial \Omega}$ is the trace operator in the Sobolev space. Problem (7.1) includes both Neumann boundary conditions (when $\mu=0$ ) and mixed (otherwise called Robin) boundary conditions (if $\mu \neq 0$ ).

For the sake of simplicity, we only consider the case of finite energy solutions, so we restrict to the range $p-1+\frac{p}{N} \leq q<p$. The weak formulation of (7.1) is the following:

$\left\{\begin{array}{l}u \in W^{1, p}(\Omega), \quad a_{0}(x, u) \in L^{1}(\Omega), \quad H(x, u, D u) \in L^{1}(\Omega), \\ \mu \int_{\partial \Omega} u \varphi d x+\int_{\Omega} a(x, u, D u) D \varphi d x+\int_{\Omega} a_{0}(x, u) \varphi d x \\ =\int_{\Omega} H(x, u, D u) \varphi d x+\int_{\partial \Omega} \omega \varphi d x \quad \text { for every } \varphi \in W^{1, p}(\Omega) \cap L^{\infty}(\Omega),\end{array}\right.$

where, with a slight abuse of notation, we still denote by $\varphi$ the trace of the test function on $\partial \Omega$.

Besides the assumptions (2.2)-(2.7), we assume that (7.2) holds with $m=$ $\frac{N(q-(p-1))}{q}$. Then, the conclusion of Theorem 3.1 remains true if $\alpha_{0}>0$, namely

Theorem 7.1. Assume (2.2), (2.3), (2.5), (2.6), (2.7) with $p-1+\frac{p}{N} \leq q<p$ and that (7.2) holds with $m=\frac{N(q-(p-1))}{q}$. Assume further that either

(i) $\alpha_{0}>0$

or

(ii) $\alpha_{0}=0, \mu>0$ and $\|f\|_{L^{m}(\Omega)}$, $\|g\|_{L^{r}(\Omega)}$ are sufficiently small.

Let $u$ be a solution of (7.1), in the sense of (7.3), such that $|u|^{\sigma} \in W^{1, p}(\Omega), \sigma=$ $\frac{(N-p)(q-(p-1))}{p(p-q)}$. Then we have

$$
\|u\|_{W^{1, p}(\Omega)}+\left\||u|^{\sigma}\right\|_{W^{1, p}(\Omega)} \leq M
$$


where $M$ depends on $p, q, N, \alpha_{0}, \alpha, \gamma,|\Omega|$ and the data $f, g, \omega$ and remains bounded when $f, g, \omega$ vary in sets which are bounded and equi-integrable, respectively, in $L^{m}(\Omega), L^{r}(\Omega)$ and $W^{1-\frac{1}{m}, m}(\partial \Omega)$. In the case that $\alpha_{0}=0$ and $\mu>0, M$ depends on $f$ and $g$ only through their norms in $L^{m}(\Omega)$ and $L^{r}(\Omega)$.

Proof. We follow the proof of Theorem 3.1 with the following variations. We use the test function $\left|T_{n}\left(G_{k}(u)\right)\right|^{\lambda-1} T_{n}\left(G_{k}(u)\right)$ and we let $n$ go to infinity, obtaining now, instead of (3.8), that

$$
\begin{aligned}
& \mu \int_{\partial \Omega}|u|\left|G_{k}(u)\right|^{\lambda} d \sigma+\alpha \lambda \int_{\Omega}|D u|^{p}\left|G_{k}(u)\right|^{\lambda-1} d x \\
& \quad+\alpha_{0} \int_{\Omega} h(|u|)\left|G_{k}(u)\right|^{\lambda} d x \\
& \leq \gamma \int_{\Omega}|D u|^{q}\left|G_{k}(u)\right|^{\lambda} d x+\int_{\Omega}|f|\left|G_{k}(u)\right|^{\lambda} d x \\
& \quad+\lambda \int_{\Omega}|g|\left|G_{k}(u)\right|^{\lambda-1} d x+\int_{\partial \Omega}\left|\omega \| G_{k}(u)\right|^{\lambda} d \sigma .
\end{aligned}
$$

Since $\int_{\partial \Omega}|z| d \sigma \leq C \int_{\Omega}(|z|+|D z|) d x$ for any $z \in W^{1,1}(\Omega)$, we obtain, using also Young's inequality,

$$
\begin{aligned}
\int_{\partial \Omega}|\omega|\left|G_{k}(u)\right|^{\lambda} d \sigma \leq & C \int_{\Omega}(|D w|+|w|)\left|G_{k}(u)\right|^{\lambda} d x \\
& +C \lambda \int_{\Omega}|w|^{p^{\prime}}\left|G_{k}(u)\right|^{\lambda-1} d x \\
& +\frac{1}{2} \alpha \lambda \int_{\Omega}|D u|^{p}\left|G_{k}(u)\right|^{\lambda-1} d x .
\end{aligned}
$$

Therefore, we deduce from (7.4)

$$
\begin{aligned}
& \frac{1}{2} \alpha \lambda \int_{\Omega}|D u|^{p}\left|G_{k}(u)\right|^{\lambda-1} d x+\alpha_{0} \int_{\Omega} h(|u|)\left|G_{k}(u)\right|^{\lambda} d x \\
& \leq \gamma \int_{\Omega}|D u|^{q}\left|G_{k}(u)\right|^{\lambda} d x+C \int_{\Omega} \tilde{f}\left|G_{k}(u)\right|^{\lambda} d x+C \lambda \int_{\Omega} \tilde{g}\left|G_{k}(u)\right|^{\lambda-1} d x,
\end{aligned}
$$

where

$$
\tilde{f}=|f|+(|D w|+|w|), \quad \tilde{g}=|g|+|w|^{p^{\prime}} .
$$

Assumption (7.2) implies that $\tilde{f} \in L^{m}(\Omega)$ and $\tilde{g} \in L^{r}(\Omega)$ (indeed, $|w|^{p^{\prime}} \in L^{r}(\Omega)$ since $\left.\frac{m^{*}}{p^{\prime}}=\frac{N(q-(p-1))}{p}=r\right)$. Therefore, we proceed henceforth as in Theorem 3.1 
to obtain

$$
\begin{aligned}
& \alpha C \int_{\Omega}\left|D\left(\left|G_{k}(u)\right|^{\sigma}\right)\right|^{p} d x+\frac{\alpha_{0}}{2} \int_{\Omega} h(|u|)\left|G_{k}(u)\right|^{\lambda} d x \\
& \leq \gamma C\left(\int_{\Omega}\left|D\left(\left|G_{k}(u)\right|^{\sigma}\right)\right|^{p}\right)^{\frac{q}{p}}\left(\int_{\Omega}\left|G_{k}(u)\right|^{\sigma p^{*}} d x\right)^{1-\frac{q}{p}} \\
& \quad+\left\|\tilde{f} \chi_{\left\{\tilde{f}>\frac{\alpha_{0}}{4} h(k)\right\}}\right\|_{L^{m}(\Omega)}\left(\int_{\Omega}\left|G_{k}(u)\right|^{\sigma p^{*}} d x\right)^{\frac{1}{m^{\prime}}} \\
& \quad+\left(\frac{\alpha_{0}}{\sqrt{h(k)}}\right)^{\lambda-1}\|\tilde{g}\|_{L^{1}(\Omega)}+\left\|\tilde{g} \chi_{\left\{\tilde{g}>\frac{\alpha_{0}^{2}}{4} \sqrt{h(k)}\right\}}\right\|_{L^{r}(\Omega)}\left(\int_{\Omega}\left|G_{k}(u)\right|^{\sigma p^{*}} d x\right)^{\frac{1}{r^{\prime}}} .
\end{aligned}
$$

If, for some $L>0$, we have $h(s) \geq s^{p-1}$ for every $s>L$, then we can easily conclude following Theorem 3.1. Indeed, since $\lambda+p-1=p \sigma$, up to taking $k>L$ we deduce in this case

$$
\alpha C \int_{\Omega}\left|D\left(\left|G_{k}(u)\right|^{\sigma}\right)\right|^{p} d x+\frac{\alpha_{0}}{2} \int_{\Omega} h(|u|)\left|G_{k}(u)\right|^{\lambda} d x \geq C\left\|\left|G_{k}(u)\right|^{\sigma}\right\|_{W^{1, p}(\Omega)}^{p} .
$$

Using the Sobolev inequality in the right-hand side we are reduced to the proof of Theorem 3.1 up to defining now $Y_{k}=\left\|\left|G_{k}(u)\right|^{\sigma}\right\|_{W^{1, p}(\Omega)}^{p}$.

If we have a general function $h(s)$, we deduce from (7.6), by Young's inequality, that

$$
\begin{aligned}
& C \int_{\Omega}\left|D\left(\left|G_{k}(u)\right|^{\sigma}\right)\right|^{p} d x+\frac{\alpha_{0}}{2} \int_{\Omega} h(|u|)\left|G_{k}(u)\right|^{\lambda} d x \\
& \leq C \int_{\Omega}\left|G_{k}(u)\right|^{\sigma p^{*}} d x+\left\|\tilde{f} \chi_{\left\{\tilde{f}>\frac{\alpha_{0}}{4} h(k)\right\}}\right\|_{L^{m}(\Omega)}\left(\int_{\Omega}\left|G_{k}(u)\right|^{\sigma p^{*}} d x\right)^{\frac{1}{m^{\prime}}} \\
& \quad+\left(\frac{\alpha_{0}}{\sqrt{h(k)}}\right)^{\lambda-1}\|\tilde{g}\|_{L^{1}(\Omega)}+\left\|\tilde{g} \chi_{\left\{\tilde{g}>\frac{\alpha_{0}^{2}}{4} \sqrt{h(k)}\right\}}\right\| \|_{L^{r}(\Omega)}\left(\int_{\Omega}\left|G_{k}(u)\right|^{\sigma p^{*}} d x\right)^{\frac{1}{r^{\prime}}} .
\end{aligned}
$$

Now we use the Poincaré-Wirtinger inequality, which implies

$$
\left\|\left|G_{k}(u)\right|^{\sigma}\right\|_{L^{p^{*}}(\Omega)} \leq \frac{1}{|\Omega|}\left\|\left|G_{k}(u)\right|^{\sigma}\right\|_{L^{1}(\Omega)}+C\left\|D\left(\left|G_{k}(u)\right|^{\sigma}\right)\right\|_{L^{p}(\Omega)},
$$

so that

$$
\int_{\Omega}\left|D\left(\left|G_{k}(u)\right|^{\sigma}\right)\right|^{p} d x \geq C\left\|\left|G_{k}(u)\right|^{\sigma}\right\|_{L^{p^{*}(\Omega)}}^{p}-C\left(\int_{\Omega}\left|G_{k}(u)\right|^{\sigma} d x\right)^{p} .
$$


We then obtain

$$
\begin{aligned}
& C\left\|\left|G_{k}(u)\right|^{\sigma}\right\|_{L^{p^{*}(\Omega)}}^{p}+\frac{\alpha_{0}}{2} \int_{\Omega} h(|u|)\left|G_{k}(u)\right|^{\lambda} d x \\
& \leq C\left(\int_{\Omega}\left|G_{k}(u)\right|^{\sigma} d x\right)^{p}+C \int_{\Omega}\left|G_{k}(u)\right|^{\sigma p^{*}} d x \\
& \quad+\left\|\tilde{f} \chi_{\left\{\tilde{f}>\frac{\alpha_{0}}{4} h(k)\right\}}\right\|_{L^{m}(\Omega)} C\left\|\left|G_{k}(u)\right|^{\sigma}\right\|_{L^{p^{*}}(\Omega)}^{\frac{p^{*}}{m^{\prime}}} \\
& \quad+\left(\frac{\alpha_{0}}{\sqrt{h(k)}}\right)^{\lambda-1}\|\tilde{g}\|_{L^{1}(\Omega)}+\left\|\tilde{g} \chi_{\left\{\tilde{g}>\frac{\alpha_{0}^{2}}{4} \sqrt{h(k)}\right\}}\right\|_{L^{r}(\Omega)}\left\|\left|G_{k}(u)\right|^{\sigma}\right\|_{L^{p^{*}}(\Omega)}^{\frac{p^{*}}{r^{\prime}}},
\end{aligned}
$$

which implies, after using Young's inequality,

$$
\begin{aligned}
& C\left\|\left|G_{k}(u)\right|^{\sigma}\right\|_{L^{p^{*}(\Omega)}}^{p}+\frac{\alpha_{0}}{2} \int_{\Omega} h(|u|)\left|G_{k}(u)\right|^{\lambda} d x \\
& \leq C\left(\int_{\Omega}\left|G_{k}(u)\right|^{\sigma} d x\right)^{p}+C\left\|\left|G_{k}(u)\right|^{\sigma}\right\|_{L^{p^{*}(\Omega)}}^{p^{*}}+M_{k},
\end{aligned}
$$

where

$$
\begin{aligned}
M_{k}= & C\left\|\tilde{f} \chi_{\left\{|\tilde{f}|>\frac{\alpha_{0}}{2} h(k)\right\}}\right\|_{L^{m}(\Omega)}^{\frac{p m^{\prime}}{p m^{\prime}-p^{*}}} \\
& +\left(\frac{\alpha_{0}}{\sqrt{h(k)}}\right)^{\lambda-1}\|\tilde{g}\|_{L^{1}(\Omega)}+C\left\|\tilde{g} \chi_{\left\{|\tilde{g}|>\frac{\alpha_{0}^{2}}{2} \sqrt{h(k)\}}\right.}\right\|_{L^{r}(\Omega)}^{\frac{p r^{\prime}}{p r^{\prime}}} .
\end{aligned}
$$

Since $\lambda \geq \sigma$ we deduce

$$
\begin{aligned}
& C\left\|\left|G_{k}(u)\right|^{\sigma}\right\|_{L^{p^{*}(\Omega)}}^{p}+\frac{\alpha_{0}}{2} h(k) \int_{\Omega}\left|G_{k}(u)\right|^{\lambda} d x \\
& \leq C\left(\int_{\Omega}\left|G_{k}(u)\right|^{\lambda} d x\right)^{\frac{p \sigma}{\lambda}}+C\left\|\left|G_{k}(u)\right|^{\sigma}\right\|_{L^{p^{*}(\Omega)}}^{p^{*}}+M_{k} .
\end{aligned}
$$

Here we set

$$
Y_{k}=\left\|\left|G_{k}(u)\right|^{\sigma}\right\|_{L^{p^{*}(\Omega)}}^{p}+\left\|\left|G_{k}(u)\right|^{\lambda}\right\|_{L^{1}(\Omega)},
$$

and the above inequality implies

$$
C_{1} Y_{k} \leq C_{2} Y_{k}^{\frac{p \sigma}{\lambda}}+C_{3} Y_{k}^{\frac{p^{*}}{p}}+M_{k} .
$$

Since $p \sigma>\lambda$, the function $\tilde{F}(Y)=C_{1} Y-C_{2} Y^{\frac{p \sigma}{\lambda}}-C_{3} Y^{\frac{p^{*}}{p}}$ is concave with maximum $F^{*}=F\left(Z^{*}\right)$; the continuity argument allows us to conclude that 
$Y_{k} \leq Z^{* 4}$. With this information in hand, going back we deduce the estimate on $\left\|D\left(\left|G_{k}(u)\right|^{\sigma}\right)\right\|_{L^{p}(\Omega)}$, hence, thanks to (7.7), the estimate of $\left|G_{k}(u)\right|^{\sigma}$ in $W^{1, p}(\Omega)$; then we can proceed as in Theorem 3.1 to conclude.

Let us now assume that $\alpha_{0}=0$ and $\mu>0$. In this case, first we split $\omega=$ $T_{\hat{k}}(\omega)+G_{\hat{k}}(\omega)$ so that

$$
\int_{\partial \Omega}|\omega|\left|G_{k}(u)\right|^{\lambda} d \sigma \leq \int_{\partial \Omega}\left|G_{\hat{k}}(\omega)\right|\left|G_{k}(u)\right|^{\lambda} d \sigma+\hat{k} \int_{\partial \Omega}\left|G_{k}(u)\right|^{\lambda} d \sigma .
$$

and choosing $\hat{k}=\frac{1}{2} \mu k$ we have

$$
\int_{\partial \Omega}|\omega|\left|G_{k}(u)\right|^{\lambda} d \sigma \leq \int_{\partial \Omega}\left|G_{\hat{k}}(\omega)\right|\left|G_{k}(u)\right|^{\lambda} d \sigma+\frac{\mu}{2} \int_{\partial \Omega}|u|\left|G_{k}(u)\right|^{\lambda} d \sigma .
$$

Therefore (7.4) implies

$$
\begin{aligned}
& \frac{\mu}{2} \int_{\partial \Omega}|u|\left|G_{k}(u)\right|^{\lambda} d \sigma+\alpha \lambda \int_{\Omega}|D u|^{p}\left|G_{k}(u)\right|^{\lambda-1} d x \\
& \leq \gamma \int_{\Omega}|D u|^{q}\left|G_{k}(u)\right|^{\lambda} d x+\int_{\Omega}|f|\left|G_{k}(u)\right|^{\lambda} d x \\
& \quad+\lambda \int_{\Omega}|g|\left|G_{k}(u)\right|^{\lambda-1} d x+\int_{\partial \Omega}\left|G_{\hat{k}}(\omega)\right|\left|G_{k}(u)\right|^{\lambda} d \sigma .
\end{aligned}
$$

Using now (7.5) with $G_{\hat{k}}(\omega)$ instead of $\omega$, we obtain

$$
\begin{aligned}
& \frac{\mu}{2} \int_{\partial \Omega}|u|\left|G_{k}(u)\right|^{\lambda} d \sigma+\frac{\alpha}{2} \lambda \int_{\Omega}|D u|^{p}\left|G_{k}(u)\right|^{\lambda-1} d x \\
& \leq \gamma \int_{\Omega}|D u|^{q}\left|G_{k}(u)\right|^{\lambda} d x+\int_{\Omega}|\tilde{f}|\left|G_{k}(u)\right|^{\lambda} d x+C \lambda \int_{\Omega}|\tilde{g}|\left|G_{k}(u)\right|^{\lambda-1} d x,
\end{aligned}
$$

where now

$$
\tilde{f}=|f|+\left(\left|D G_{\hat{k}}(w)\right|+\left|G_{\hat{k}}(w)\right|\right), \quad \tilde{g}=|g|+\left|G_{\hat{k}}(w)\right|^{p^{\prime}} .
$$

Then, we proceed as before and we get

$$
\begin{aligned}
& \frac{\mu}{2} \int_{\partial \Omega}|u|\left|G_{k}(u)\right|^{\lambda} d \sigma+C \int_{\Omega}\left|D\left(\left|G_{k}(u)\right|^{\sigma}\right)\right|^{p} d x \\
& \leq C \int_{\Omega}\left|G_{k}(u)\right|^{\sigma p^{*}} d x+\|\tilde{f}\|_{L^{m}(\Omega)}\left(\int_{\Omega}\left|G_{k}(u)\right|^{\sigma p^{*}} d x\right)^{\frac{1}{m^{\prime}}} \\
& \quad+\|\tilde{g}\|_{L^{r}(\Omega)}\left(\int_{\Omega}\left|G_{k}(u)\right|^{\sigma p^{*}} d x\right)^{\frac{1}{r^{\prime}}} .
\end{aligned}
$$

${ }^{4}$ In particular, this implies that $\left\|G_{k}(u)^{\lambda}\right\|_{L^{1}(\Omega)} \leq Z^{*}$. Hence, using the fact that $h(k) \rightarrow \infty$ as $k \rightarrow \infty$, we could now get rid of the first term in the right-hand side of (7.8) and recover our usual inequality

$$
C_{1}\left\|\left|G_{k}(u)\right|^{\sigma}\right\|_{L^{p^{*}}(\Omega)}^{p} \leq C_{3}\left\|\left|G_{k}(u)\right|^{\sigma}\right\|_{L^{p^{*}}(\Omega)}^{p^{*}}+M_{k} .
$$


We can now use another Poincaré-type inequality, namely that

$$
\|z\|_{L^{s}(\Omega)} \leq C\left(\|D u\|_{L^{s}(\Omega)}+\|u\|_{L^{s}(\partial \Omega)}\right) .
$$

Using (7.10) with $s=1$ and combined with the Poincaré-Wirtinger inequality this implies

$$
\|z\|_{L^{p}(\Omega)} \leq C\left(\|D z\|_{L^{p}(\Omega)}+\|z\|_{L^{1}(\Omega)}\right) \leq C\left(\|D z\|_{L^{p}(\Omega)}+\|z\|_{L^{1}(\partial \Omega)}\right) .
$$

Applied to $z=\left|G_{k}(u)\right|^{\sigma}$, and together with the Sobolev inequality, it gives

$$
\left\|\left|G_{k}(u)\right|^{\sigma}\right\|_{L^{p^{*}}(\Omega)} \leq C\left\|\left|G_{k}(u)\right|^{\sigma}\right\|_{L^{1}(\partial \Omega)}+C\left\|D\left|G_{k}(u)\right|^{\sigma}\right\|_{L^{p}(\Omega)},
$$

so that

$$
\int_{\Omega}\left|D\left(\left|G_{k}(u)\right|^{\sigma}\right)\right|^{p} d x \geq C\left\|\left|G_{k}(u)\right|^{\sigma}\right\|_{L^{p^{*}(\Omega)}}^{p}-C\left(\int_{\partial \Omega}\left|G_{k}(u)\right|^{\sigma} d \gamma\right)^{p} .
$$

Therefore, (7.9) implies

$$
\begin{aligned}
& \frac{\mu}{2} k \int_{\partial \Omega}\left|G_{k}(u)\right|^{\lambda} d \sigma+C\left\|\left|G_{k}(u)\right|^{\sigma}\right\|_{L^{p^{*}}(\Omega)}^{p} \\
& \leq C\left(\int_{\partial \Omega}\left|G_{k}(u)\right|^{\sigma} d \gamma\right)^{p}+C \int_{\Omega}\left|G_{k}(u)\right|^{\sigma p^{*}} d x \\
& \quad+\|\tilde{f}\|_{L^{m}(\Omega)}\left(\int_{\Omega}\left|G_{k}(u)\right|^{\sigma p^{*}} d x\right)^{\frac{1}{m^{\prime}}}+\|\tilde{g}\|_{L^{r}(\Omega)}\left(\int_{\Omega}\left|G_{k}(u)\right|^{\sigma p^{*}} d x\right)^{\frac{1}{r^{\prime}}} .
\end{aligned}
$$

Henceforth, we conclude as in the previous case, using that $\lambda \geq \sigma$, and defining now

$$
Y_{k}=\int_{\partial \Omega}\left|G_{k}(u)\right|^{\lambda} d \sigma+\left\|\left|G_{k}(u)\right|^{\sigma}\right\|_{L^{p^{*}(\Omega)}}^{p} .
$$

Observe that $\|\tilde{f}\|_{L^{m}(\Omega)}$ and $\|\tilde{g}\|_{L^{r}(\Omega)}$ are sufficiently small provided $\|f\|_{L^{m}(\Omega)}$ and $\|g\|_{L^{r}(\Omega)}$ are sufficiently small and $\hat{k}$ is large enough, i.e. $k$ is large enough. In this way no smallness condition is assumed on $\omega$. Then, we conclude the a priori estimate in the usual way.

Let us notice that, as in Section 3, the proof should be slightly modified if $\alpha_{0}>0$ and $\mu>0$ in order to deal with the limiting case $q=p-1+\frac{p}{N}$; however the boundary term can be dealt with in a similar way as above and this case is also reduced to the proof given for Theorem 3.1.

Remark 7.2. With the same arguments, it is possible to deal with the case where the boundary condition is in the form

$$
a(x, u, D u) \cdot v+\mu \beta(x, u)=\omega \quad \text { on } \partial \Omega,
$$

where $\beta(x, s)$ is nondecreasing with respect to $s$ and tends to infinity as $|s| \rightarrow \infty$. 
Finally, once we have established an a priori estimate, we can also deduce an existence result as in Theorem 3.7. Namely, assuming (2.2)-(2.7) and (3.34), (3.35), with $p-1+\frac{p}{N} \leq q<p$, and in addition that (7.2) holds true, then there exists a solution $u$ of (7.1) in the sense of (7.3) satisfying the condition $|u|^{\sigma} \in W^{1, p}(\Omega)$ if either $\alpha_{0}>0$, or $\alpha_{0}=0, \mu>0$ and $\|f\|_{L^{m}(\Omega)},\|g\|_{L^{r}(\Omega)}$ are sufficiently small.

\subsection{Data in Lorentz spaces}

Without significant changes, one can extend Theorem 3.1 to the case that $f$ and $g$ belong to suitable Lorentz spaces $L^{p, q}(\Omega)$. Recall that $v \in L^{p, q}(\Omega)$ if (see e.g. [31])

$$
\int_{0}^{\infty}\left(v^{*}(s) s^{\frac{1}{q}}\right)^{p} \frac{d s}{s}<\infty,
$$

where $v^{*}$ is the decreasing rearrangement of $v$.

In the case when $p-1+\frac{p}{N} \leq q<p$, one can take

$$
\begin{array}{ll}
f \in L^{m, s}(\Omega) & \text { with } m=\frac{N(q-(p-1))}{q} \text { and } s=\frac{m(N-p)}{N-p m}, \\
g \in L^{r, t}(\Omega) & \text { with } r=\frac{N(q-(p-1))}{p} \text { and } t=\frac{r(N-p)}{N-p r} .
\end{array}
$$

The only change in the proof is that we use the improved Sobolev inequality

$$
\|v\|_{L^{p^{*}, p}(\Omega)} \leq C_{S}\|D v\|_{L^{p}(\Omega)}, \quad \forall v \in W_{0}^{1, p}(\Omega) .
$$

Therefore we estimate

$$
\begin{aligned}
\int_{\Omega}\left|f \| G_{k}(u)\right|^{\lambda} d x & \leq\|f\|_{L^{m, s}(\Omega)}\left\|\left|G_{k}(u)\right|^{\lambda}\right\|_{L^{m^{\prime}, s^{\prime}}(\Omega)} \\
& =\|f\|_{L^{m, s}(\Omega)}\left\|\left|G_{k}(u)\right|\right\|_{L^{\lambda m^{\prime}, \lambda s^{\prime}}(\Omega)} \\
& \leq\|f\|_{L^{m, s}(\Omega)}\left\|G_{k}(u)^{\lambda}\right\|_{W_{0}^{1, p}(\Omega)},
\end{aligned}
$$

as soon as $\lambda m^{\prime}=p^{*}$ and $\lambda s^{\prime}=p$. This gives the link $m=\frac{N(q-(p-1)}{q}$ already known, and, since $\frac{m^{\prime}}{s^{\prime}}=\frac{p^{*}}{p}$, the value for $s$. Similarly one has

$$
\begin{aligned}
\int_{\Omega}|g|\left|G_{k}(u)\right|^{\lambda-1} d x & \leq\|g\|_{L^{r, t}(\Omega)}\left\|\left|G_{k}(u)\right|^{\lambda-1}\right\|_{L^{r^{\prime}, t^{\prime}}(\Omega)} \\
& =\|g\|_{L^{r, t}(\Omega)}\left\|G_{k}(u)^{\lambda}\right\|_{L^{\frac{\lambda-1}{\lambda}}}^{\frac{(\lambda-1)}{\lambda} r^{\prime}, \frac{(\lambda-1)}{\lambda} t^{\prime}(\Omega)} \\
& \leq\|g\|_{L^{r, t}(\Omega)}\left\|G_{k}(u)^{\lambda}\right\|_{W_{0}^{1, p}(\Omega)}^{\frac{\lambda-1}{\lambda}},
\end{aligned}
$$

as soon as $\frac{(\lambda-1)}{\lambda} r^{\prime}=p^{*}$ and $\frac{(\lambda-1)}{\lambda} t^{\prime}=p$. This gives the values of $r, t$ in (7.11) above. 
Note that, as expected, when $q=p-1+\frac{p}{N}$, i.e. $m=\left(p^{*}\right)^{\prime}$ and $r=1$, we find $s=p^{\prime}\left(f \in L^{\left(p^{*}\right)^{\prime}, p^{\prime}}(\Omega)\right)$ and $t=1\left(g \in L^{1}(\Omega)\right)$.

In the case that $\alpha_{0}>0$, the inequalities above are used with $f$ and $g$ replaced, as usual, by $f \chi_{\left\{|f|>a_{k}\right\}}$ and $g \chi_{\left\{|g|>b_{k}\right\}}$ for some $a_{k}, b_{k} \rightarrow \infty$. Since all the exponents are finite, the Lorentz norm of $v \chi_{\{|v|>k\}}$ becomes small as $k \rightarrow \infty$, and we can still follow the steps of Theorem 3.1.

In the case when $\frac{N(p-1)}{N-1}<q<p-1+\frac{p}{N}$, the term $g$ should always be taken in $L^{1}(\Omega)$, and one cannot improve this regularity in Lorentz spaces, while $f$ could be taken again in $L^{m, s}(\Omega)$ with $m=\frac{N(q-(p-1))}{q}$ and $s=\frac{m(N-p)}{N-p m}$. Note that both exponents are still strictly larger than 1 . However, let us recall that solutions have not finite energy in this range of growth and one should suitably modify the statements and the proofs as we did in Section 4.

\section{References}

[1] N. AlAA and M. PIERRE, Weak solutions of some quasilinear elliptic equations with data measures, SIAM J. Math. Anal. 24 (1993), 23-35.

[2] A. Alvino, V. FERONE and A. MERCALDO, Sharp a priori estimates for a class of nonlinear elliptic equations with lower order term, Ann. Mat. Pura Appl., to appear.

[3] A. Alvino, P. L. Lions and G. Trombetti, Comparison results for elliptic and parabolic equations via Schwarz symmetrization, Ann. Inst. H. Poincaré Anal. Non Linéaire 7 (1990), 37-65.

[4] G. BARles and F. DA Lio, On the generalized Dirichlet problem for viscous HamiltonJacobi equations, J. Math. Pures Appl. 83 (2004), 53-75.

[5] G. BARLES and A. PORRETTA, Uniqueness for unbounded solutions to stationary viscous Hamilton-Jacobi equations, Ann. Sc. Norm. Super. Pisa Cl. Sci. (5) 5 (2006), 107-136.

[6] P. Bénilan, L. Boccardo, T. Gallouët, R. Gariepy, M. Pierre and J. L. VÁZQUEZ, An $L^{1}$ theory of existence and uniqueness of solutions of nonlinear elliptic equations, Ann. Scuola Norm. Sup. Pisa Cl. Sci. (4) 22 (1995), 241-273.

[7] A. Bensoussan, L. BocCARDO and F. MURAT, On a nonlinear partial differential equation having natural growth terms and unbounded solution, Ann. Inst. H. Poincaré Anal. Non Linéaire 5 (1988), 347-364.

[8] M. F. Betta, A. Mercaldo, F. Murat and M. M. Porzio, Existence of renormalized solutions to nonlinear elliptic equations with a lower-order term and right-hand side a measure, J. Math. Pures Appl. 82 (2003), 90-124.

[9] L. BocCARDo, Some nonlinear Dirichlet problems in $L^{1}$ involving lower order terms in divergence form, In: "Progress in Elliptic and Parabolic Partial Differential Equations", 1994, A. Alvino, P. Buonocore, V. Ferone, E. Giarrusso, S. Matarasso, R. Toscano and G. Trombetti (eds.), Pitman Res. Notes Math. Ser., Vol. 350, Longman, Harlow, 1996, 43-57.

[10] L. BOCCARDO and D. GIACHETTI, Alcune osservazioni sulla regolarità delle soluzioni di problemi fortemente non lineari e applicazioni, Ricerche Mat. 14 (1989), 309-323.

[11] L. Boccardo, D. Giachetti, J. I. Diaz and F. Murat, Existence and regularity of renormalized solutions for some elliptic problems involving derivatives of nonlinear terms, J. Differential Equations 106 (1993), 215-237.

[12] L. BocCARDO and T. GALlOUËT, Nonlinear elliptic equations with right-hand side measures, Comm. Partial Differential Equations 17 (1992), 641-655. 
[13] L. BocCARdo, T. GALlouËT and L. ORsina Existence and uniqueness of entropy solutions for nonlinear elliptic equations with measure data, Ann. Inst. H. Poincaré Anal. Non Linéaire 13 (1996), 539-551.

[14] L. BOCCARDO and F. MURAT, Almost everywhere convergence of the gradients of solutions to elliptic and parabolic equations, Nonlinear Anal. 19 (1992), 581-597.

[15] L. Boccardo, F. Murat and J.-P. PUel Existence de solutions faibles pour des équations elliptiques quasi-linéaires à croissance quadratique, In: "Nonlinear partial differential equations and their applications", Collège de France Seminar, Vol. IV, H. Brezis and J.-L. Lions (eds.), Pitman Research Notes in Mathematics, Vol. 84, London, 1983, 19-73.

[16] L. BocCARDO, F. MURAT and J.-P. PUEL, Existence of bounded solutions for nonlinear elliptic unilateral problems, Ann. Mat. Pura Appl. 152 (1988), 183-196.

[17] L. BocCARdo, F. MURAT and J.-P. PUEL, $L^{\infty}$ estimates for some nonlinear partial differential equations and an application to an existence result, SIAM J. Math. Anal. 23 (1992), $326-333$

[18] L. BocCARdo and M. M. PorzIo, Quasilinear elliptic equations with subquadratic growth, J. Differential Equations 229 (2006), 367-388.

[19] G. BotTaro and M. E. Marina, Problema di Dirichlet per equazioni ellittiche di tipo variazionale su insiemi non limitati, Boll. Un. Mat. Ital. (4) 8 (1973), 46-56.

[20] I. Capuzzo Dolcetta, F. LeONi and A. Porretta, Hölder estimates for degenerate elliptic equations with coercive Hamiltonians. Trans. Amer. Math. Soc. 362 (2010), 45114536.

[21] A. Dall' Aglio, D. Giachetti and J.-P. Puel, Nonlinear elliptic equations with natural growth in general domains, Ann. Mat. Pura Appl. 181 (2002), 407-426.

[22] G. Dal Maso, F. Murat, L. Orsina and A. Prignet, Renormalized solutions of elliptic equations with general measure data, Ann. Scuola Norm. Sup. Pisa Cl. Sci. (4) 28 (1999), 741-808.

[23] T. DEL VECCHIO and M. M. PORZIO, Existence results for a class of non-coercive Dirichlet problems, Ricerche Mat. 44 (1995), 421-438 (1996).

[24] V. FERONE and B. Messano, Comparison and existence results for classes of nonlinear elliptic equations with general growth in the gradient, Adv. Nonlinear Stud. 7 (2007), 3146.

[25] V. FERONE and F. MURAT, Quasilinear problems having quadratic growth in the gradient: an existence result when the source term is small, In: "Equations aux dérivées partielles et applications", articles dédiés à Jacques-Louis Lions, Gauthier-Villars, Paris, 1998, 497-515.

[26] V. FERONE and F. MURAT, Nonlinear problems having natural growth in the gradient: an existence result when the source terms are small, Nonlinear Anal. 42 (2000), 1309-1326.

[27] V. Ferone, M. R. Posteraro and J. M. Rakotoson, $L^{\infty}$-estimates for nonlinear elliptic problems with p-growth in the gradient, J. Inequal. Appl. 3 (1999), 109-125.

[28] M. FuKUSHIMA, K. SATO and S. TANIGUCHI, On the closable part of pre-Dirichlet forms and the fine support of the underlying measures, Osaka J. Math. 28 (1991), 517-535.

[29] N. Grenon, F. MURAT and A. Porretta, Existence and a priori estimate for elliptic problems with subquadratic gradient dependent terms, C. R. Math. Acad. Sci. Paris 342 (2006), 23-28.

[30] K. HANSSON, V. MAZ'YA and I. VERBITSKY, Criteria of solvability for multidimensional Riccati equation, Ark. Mat. 37 (1999), 87-120.

[31] R. Hunt, On L ( $p, q)$ spaces, Enseign. Math 12 (1966), 249-276.

[32] C. LEONE and A. PorrettA, Entropy solutions for nonlinear elliptic equations in $L^{1}$, Nonlinear Anal. 32 (1998), 325-334.

[33] J. LERAY and J.-L. LiONS, Quelques résultats de Višik sur les problèmes elliptiques non linéaires par les méthodes de Minty-Browder, Bull. Soc. Math. France 93 (1965), 97-107.

[34] P.-L. Lions, Résolution de problèmes elliptiques quasilinéaires, Arch. Ration. Mech. Anal. 74 (1980), 335-353. 
[35] P.-L. Lions, Quelques remarques sur les problèmes elliptiques quasilinéaires du second ordre, J. Anal. Math. 45 (1985), 234-254.

[36] P.-L. LiOns and F. MURAT, Solutions renormalisées d'équations elliptiques non linéaires, unpublished.

[37] C. MAderna, C. D. PAGANI and S. SAlsa, Quasilinear elliptic equations with quadratic growth in the gradient, J. Differential Equations 97 (1992), 54-70.

[38] F. MURAT, Soluciones renormalizadas de EDP elipticas no lineales, Preprint 93023, Laboratoire d'Analyse Numérique, Université Paris VI, (1993), 38 pages.

[39] A. PORRETTA, Some remarks on the regularity of solutions for a class of elliptic equations with measure data, Houston J. Math. 26 (2000), 183-213.

[40] A. PORRETTA, On the comparison principle for p-Laplace type operators with first order terms, In: "On the notions of solution to nonlinear elliptic problems: results and developments", Quaderni di Matematica, Vol. 23, Department of Mathematics, Seconda Università di Napoli, Caserta, 2008, 459-497.

[41] G. StampaCchia, Le problème de Dirichlet pour les équations elliptiques du second ordre à coefficients discontinus, Ann. Inst. Fourier (Grenoble) 15 (1965), 189-258.

Centre Universitaire de Bourges

Rue Gaston Berger, BP 4043

18028 Bourges cedex, France

nathalie.grenon@univ-orleans.fr

Laboratoire Jacques-Louis Lions Université Pierre et Marie Curie

Boîte courrier 187

75252 Paris cedex 05, France

murat@ann.jussieu.fr

Dipartimento di Matematica Università di Roma Tor Vergata Via della Ricerca Scientifica, 1 00133 Roma, Italia porretta@axp.mat.uniroma2.it 